NBER WORKING PAPER SERIES

\title{
ORIGINS OF THE OPIOID CRISIS AND ITS ENDURING IMPACTS
}

\author{
Abby E. Alpert \\ William N. Evans \\ Ethan M.J. Lieber \\ David Powell \\ Working Paper 26500 \\ http://www.nber.org/papers/w26500 \\ NATIONAL BUREAU OF ECONOMIC RESEARCH \\ 1050 Massachusetts Avenue \\ Cambridge, MA 02138 \\ November 2019
}

We thank Mireille Jacobson, Rosalie Liccardo Pacula, Harold Pollack and seminar and conference participants at Illinois, Notre Dame, RAND, Temple, Tulane, USC, Health and Labor Market Effects of Public Policy at UCSB, iHEA, Midwest Health Economics Conference, and NBER Summer Institute Crime Meeting for helpful feedback. For help obtaining some of the unsealed court documents, we also thank Caitlin Esch and Marketplace, Judge Booker T. Stephens of West Virginia, Nicholas Weilhammer in the Office of Public Records for the Office of the Attorney General of Florida, and La Dona Jensen in the Office of the Attorney General of Washington. Powell gratefully acknowledges financial support from NIDA (1R21DA041653 and P50DA046351). Evans gratefully acknowledges financial support from the Institute for Scholarship in the Liberal Arts at the University of Notre Dame. The views expressed herein are those of the authors and do not necessarily reflect the views of the National Bureau of Economic Research.

NBER working papers are circulated for discussion and comment purposes. They have not been peer-reviewed or been subject to the review by the NBER Board of Directors that accompanies official NBER publications.

(C) 2019 by Abby E. Alpert, William N. Evans, Ethan M.J. Lieber, and David Powell. All rights reserved. Short sections of text, not to exceed two paragraphs, may be quoted without explicit permission provided that full credit, including (C) notice, is given to the source. 
Origins of the Opioid Crisis and Its Enduring Impacts

Abby E. Alpert, William N. Evans, Ethan M.J. Lieber, and David Powell

NBER Working Paper No. 26500

November 2019

JEL No. I12,I18

\begin{abstract}
$\underline{\text { ABSTRACT }}$
Overdose deaths involving opioids have increased dramatically since the mid-1990s, leading to the worst drug overdose epidemic in U.S. history, but there is limited empirical evidence on the initial causes. In this paper, we examine the role of the 1996 introduction and marketing of OxyContin as a potential leading cause of the opioid crisis. We leverage cross-state variation in exposure to OxyContin's introduction due to a state policy that substantially limited OxyContin's early entry and marketing in select states. Recently-unsealed court documents involving Purdue Pharma show that state-based triplicate prescription programs posed a major obstacle to sales of OxyContin and suggest that less marketing was targeted to states with these programs. We find that OxyContin distribution was about 50\% lower in "triplicate states" in the years after the launch. While triplicate states had higher rates of overdose deaths prior to 1996, this relationship flipped shortly after the launch and triplicate states saw substantially slower growth in overdose deaths, continuing even twenty years after OxyContin's introduction. Our results show that the introduction and marketing of OxyContin explain a substantial share of overdose deaths over the last two decades.
\end{abstract}

Abby E. Alpert

The Wharton School

University of Pennsylvania

3641 Locust Walk

Philadelphia, PA 19104

and NBER

alpertab@wharton.upenn.edu

William N. Evans

Keough-Hesburgh Professor of Economics

Department of Economics

University of Notre Dame

3111 Jenkins Nanovic Halls

Notre Dame, IN 46556-7000

and NBER

wevans1@nd.edu
Ethan M.J. Lieber

Department of Economics

University of Notre Dame

3049 Jenkins Nanovic Halls

Notre Dame, IN 46556

and NBER

elieber@nd.edu

David Powell

RAND Corporation

1776 Main Street

P.O. Box 2138

Santa Monica, CA 90407

David_Powell@rand.org 


\section{$1 \quad$ Introduction}

Over the last two decades, there has been a staggering increase in mortality from drug overdoses in the U.S. Between 1983 and 2017, the drug overdose death rate increased by a factor of eight with a noticeable inflection point in the mid-1990s, as shown in Figure 1.

Overdose deaths involving opioids are the primary driver of this increase, accounting for $75 \%$ of the growth and, by 2017, two-thirds of all drug overdose deaths were related to opioids. Opioid overdoses claimed the lives of 47,600 people in 2017 (Scholl et al., 2019) and almost 400,000 since $1999,{ }^{1}$ about the same number of U.S. soldiers that died in World War II (DeBruyne, 2018). This massive rise in opioid deaths has contributed to the longest sustained decline in life expectancy since 1915 (Dyer, 2018).

There are many hypotheses about the initial causes of the opioid crisis. Case and Deaton $(2015,2017)$ suggest that demand factors played an important role as worsening cultural and economic conditions may have sparked a surge in "deaths of despair": suicides, alcohol-related mortality, and drug overdoses. Alternative hypotheses, though not mutually exclusive, consider the role of supply factors such as the dramatic increase in opioid access driven by changing physician attitudes and practice patterns. Beginning in the $1990 \mathrm{~s}$, doctors began to treat pain more aggressively with opioids (Jones et al., 2018), following widespread concerns that pain had been "under-treated" (Morgan, 1985; WHO, 1986; Melzack, 1990). The American Pain Society launched an influential campaign declaring pain as the "fifth vital sign" and, in response, the Joint Commission on Accreditation of Healthcare Organizations (JCAHO) revised its guidelines in 2001, requiring that doctors assess pain along with other vitals during medical visits (Phillips, 2000). In addition, the introduction and aggressive marketing of Purdue Pharma's OxyContin in 1996 has also been implicated as a central cause of the opioid crisis (Van Zee, 2009; Kolodny et al., 2015; Quinones, 2015). ${ }^{2}$

Despite the discussion of these hypotheses throughout the literature, there is surprisingly little empirical evidence on any individual factor's importance. Existing studies have considered underlying factors that may be driving opioid misuse such as economic conditions and labor demand shocks (Hollingsworth et al., 2017; Ruhm, 2019; Betz and Jones, 2018; Charles et al.,

\footnotetext{
${ }^{1}$ https://www.cdc.gov/drugoverdose/epidemic/index.html

${ }^{2}$ As of June 2019, 48 states and 500 cities have filed lawsuits against Purdue Pharma for its deceptive marketing practices claiming that they have contributed to the opioid crisis (https://www.vanityfair.com/news/2019/06/davidsackler-pleads-his-case-on-the-opioid-epidemic).
} 
2019; Pierce and Schott, forthcoming). Other studies have tested whether increased access to opioids, through prescriptions to family members (Khan et al., 2019) or encounters with physicians with high propensities to prescribe opioids (Barnett et al., 2017), predict long-term use of opioids. This research is relevant to understanding the role of supply versus demand factors in driving the ongoing opioid crisis; however, none of these studies evaluate the causes of the initial rise in opioid deaths in the mid-1990s. Moreover, these studies often find effects which are too small to explain the massive growth in opioid deaths.

In this paper, we provide the first quasi-experimental evidence on the initial causes of the opioid crisis. We examine the role of the introduction and marketing of OxyContin as a potential leading cause, exploring its impacts on drug overdose deaths over the two decades since its launch. OxyContin is a prescription opioid pain reliever whose active ingredient, oxycodone, has been in use in the U.S. since the early 1900s. OxyContin's key technological innovation was its sustained-release formulation that utilizes a high concentration of the active ingredient to provide 12 hours of continuous pain relief. However, the timed-release aspect of OxyContin is contingent on taking the pill whole. Crushing or dissolving the pill allowed users to access the high dosage of oxycodone all at once, producing an intense high. OxyContin quickly became one of the leading drugs of abuse in the U.S. (Cicero et al., 2005) and concerns about widespread abuse of this drug were being reported by 2000 (GAO, 2003).

Since OxyContin was launched nationwide, it is difficult to isolate its effects from other concurrent changes to prescribing practice patterns, opioid availability, and demand. We address this issue by exploiting geographic variation in exposure to OxyContin's introduction due to a previously unexplored state policy that substantially limited OxyContin's entry and marketing in select states. Information on the importance of this state policy was obtained from recentlyunsealed court documents that we collected from multiple settled lawsuits and investigations involving Purdue Pharma. These documents provide an unprecedented look at the manufacturer's internal marketing strategies around the introduction of OxyContin. They reveal that Purdue Pharma viewed state-based "triplicate prescription programs," an unusually stringent early prescription drug monitoring program that required the use of special state-issued prescription forms for Schedule II opioids, as a significant barrier to prescribing OxyContin. As a result, they suggested that the company should not target marketing to states with these programs because of the lower expected returns. 
Discussions of triplicate programs appear frequently throughout the internal documents concerning the launch and promotion of OxyContin. Purdue Pharma's focus group research found that these programs had a chilling effect on the prescribing of Schedule II opioids such as oxycodone. Doctors in triplicate states rarely used Schedule II opioids because "writing triplicate prescriptions was more trouble than others" and providers "did not want to give the Government an excuse to monitor their activities" (Groups Plus, 1995). They found that "the doctors in the triplicate states were not enthusiastic about the product [OxyContin] at all, with only a couple indicating they would ever use it, and then in very infrequent situations" (Groups Plus, 1995). The research concluded that doctors in these states were unlikely to adopt OxyContin, leading to the recommendation that "the product [OxyContin] should only be positioned to physicians in non-triplicate states" (Groups Plus, 1995).

Using a difference-in-differences framework, we take advantage of the variation in OxyContin supply induced by the triplicate policies to study drug overdose trends in states with triplicate programs (henceforth "triplicate states") relative to states without these programs ("non-triplicate states"). We consider triplicate states less exposed to OxyContin's introduction for two reasons: first, there appears to have been less initial marketing targeted to these states and, second, the triplicate programs themselves were a barrier to prescribing OxyContin. Together, these two forces provide a source of exogenous geographic variation in exposure to OxyContin's introduction. We will also aim to disentangle these two mechanisms.

Consistent with our hypothesis, we find that OxyContin distribution was more than twice as high in non-triplicate states in the years after the launch. When we compare this to hydrocodone distribution, another commonly abused opioid that was not subject to triplicate policies because it was largely classified as a Schedule III drug, we find almost identical levels of hydrocodone distribution across triplicate and non-triplicate states. Additionally, we find much higher rates of OxyContin misuse in non-triplicate states relative to triplicate states, but similar rates of misuse of all other pain relievers. These results are consistent with differences in overdose death rates being primarily attributable to OxyContin.

Given this variation in OxyContin exposure due to triplicate policies, we turn to estimating OxyContin's impacts on the time path of drug overdose deaths over the short and long run. Figure 2 shows the raw trends in drug overdose deaths per 100,000 people comparing triplicate and non-triplicate states. Prior to OxyContin's introduction, the two groups of states 
were trending similarly, although non-triplicate states had lower rates of drug overdose deaths. This relationship flipped shortly after OxyContin's launch as drug overdose deaths increased much more rapidly in non-triplicate states than in triplicate states, a trend that continued even twenty years later. We find that this differential growth is driven almost entirely by drug overdoses involving prescription opioids until 2010. After 2010, when the original formulation of OxyContin was removed from the market and replaced with an abuse-deterrent version, large differences in overdose deaths involving heroin and synthetic opioids emerged across triplicate and non-triplicate states. This is consistent with prior evidence that areas with early exposure to OxyContin experienced differential transitions to illicit opioids post-reformulation as people substituted from OxyContin to heroin (Alpert et al., 2018; Evans et al., 2019). Overall, our estimates imply that non-triplicate states would have had an average of $36 \%$ fewer drug overdose deaths and 44\% fewer opioid overdose deaths in 1996-2017 if they had been triplicate states.

Although there were many changes to the opioid environment over this time period, our results are not explained by adoption of other opioid policies, misuse of other opioid drugs, or economic shocks targeted differentially to non-triplicate states. We find that differences in stateand county-level characteristics such as urbanicity and population size between triplicate and non-triplicate states also do not explain differential overdose mortality growth. In permutation tests, we show that it is statistically rare to observe overdose growth differences between triplicate and non-triplicate states of a similar magnitude when we randomly assign triplicate status to other combinations of states, suggesting that triplicate states experienced uniquely low growth.

This research contributes to our understanding of what initially sparked the opioid crisis. We demonstrate that the introduction and marketing of OxyContin explains a substantial share of overdose deaths over the last two decades. Although triplicate programs were discontinued in the years after OxyContin's launch, their initial deterrence of OxyContin promotion and adoption had long-term effects on overdose deaths in these states, dramatically decreasing overdose death rates even today. The triplicate states we study, spread throughout the U.S., currently have some of the lowest overdose death rates in the country. Our work, therefore, also speaks to the substantial geographic variation in overdose deaths. Within small regions of the country, there are widely-varying drug overdose death rates and this variation is difficult to explain based on demographics, economic conditions, and current policies. Our results suggest the importance of 
initial conditions - particularly the policy landscape at the beginning of the epidemic - in inducing variation in overdose rates that persists even decades later.

Finally, our results demonstrate the potentially harmful consequences of pharmaceutical promotion for controlled substances. Our analysis of mechanisms finds that while triplicate programs themselves may have independently discouraged OxyContin adoption, the evidence also suggests that the relative lack of marketing in these states played an important independent role in reducing exposure to the drug. When triplicate states are compared to other states with similar initial prescribing practices or even states which had eliminated their triplicate programs just prior to 1996, they still have uniquely low growth in overdose deaths. We discuss how this evidence is consistent with marketing practices playing a central role in explaining trends in drug overdose deaths.

The remainder of the paper proceeds as follows. We provide additional background in Section 2. Section 3 introduces the data while Section 4 discusses the empirical strategy. We present the results in Section 5. In Section 6, we discuss the mechanisms for our results, isolating the effects of triplicate programs and marketing. Section 7 concludes.

\section{$2 \quad$ Background}

\subsection{OxyContin's Launch and Promotional Activities}

OxyContin is a long-acting formulation of oxycodone, a morphine-like drug, produced by Purdue Pharma. It is classified as a Schedule II controlled substance given its high potential for abuse. The Food and Drug Administration (FDA) approved OxyContin in 1995 and the drug was introduced to the market in January 1996. OxyContin entered the market as Purdue Pharma's patent for MS Contin - a long-acting form of morphine used for treating late-stage cancer pain — was set to expire. Purdue Pharma aimed to replace MS Contin with OxyContin as well as to expand into additional markets: patients in the earlier stages of cancer (positioning OxyContin as "the opioid to start with and to stay with") and the much larger market for noncancer pain. Prior to OxyContin's launch, patients with non-cancer pain would have been typically treated (if at all) with non-opioid painkillers (e.g., Tylenol) or short-acting combination 
products that combine much smaller doses of either oxycodone or hydrocodone with acetaminophen (e.g., Percocet, Tylox, Vicodin). ${ }^{3}$

OxyContin's initial marketing strategy centered on claims that the drug had low abuse potential and was safer than other opioid drugs, which would later prove to be false. The original FDA-approved product label for OxyContin included the statement that "delayed absorption as provided by OxyContin tablets, is believed to reduce the abuse liability of a drug." Additionally, marketing materials relied heavily on a 100-word letter to the editor in the New England Journal of Medicine (Porter and Jick, 1980) to support the claim that the risk of addiction among opioid users was "much less than one percent." Some marketing materials failed to include any information about its addiction potential (Van Zee, 2009). These misinformed or misleading claims were important for convincing doctors who had been cautious about prescribing opioids to switch from less potent painkillers to OxyContin for treating noncancer pain. To achieve growth for non-cancer chronic pain - a previously untapped market for opioids-Purdue Pharma also heavily targeted marketing to primary care physicians, although this raised concerns given their limited experience and training in pain management. From 1997 to 2002, OxyContin prescriptions increased at a faster rate for non-cancer pain than for cancer pain (GAO, 2003).

In 2001, the FDA product label for OxyContin was revised to remove the incorrect statements about its abuse liability and to add a black box safety warning. However, the indication was also changed from covering patients "where use of an opioid analgesic is appropriate for more than a few days" to those who require "a continuous around-the-clock analgesic for an extended period of time." This may have further expanded the market for chronic pain. Internal documents show that Purdue Pharma believed that the new label "created enormous opportunities" and "in effect, the FDA has expanded the indication for OxyContin." They further noted that "this broad labeling is likely to never again be available for an opioid seeking FDA approval" (Purdue Pharma Budget Plan, 2002, p. 47).

Purdue Pharma's advertising campaign was unusually aggressive for a prescription drug and unprecedented for an opioid. The promotional budget between 1996 and 2001 for

\footnotetext{
${ }^{3}$ The dosage of the combination oxycodone and hydrocodone products is limited by the maximum safe dosage of acetaminophen (which can cause liver failure at high dosages). In contrast, OxyContin is made of pure oxycodone, so there is no ceiling dosage (GAO, 2003). This purity allows OxyContin to be used at much higher dosages to treat more severe levels of pain than the combination products.
} 
OxyContin was six- to twelve-times more than they had spent on advertising for MS Contin during its first six years on the market, and what Janssen Pharmaceutical Products spent for the promotion of Duragesic, one of OxyContin's competitors (GAO, 2003). They employed an enormous sales force to promote the drug to doctors which doubled in size between 1996 and 2002. ${ }^{4}$ Additionally, Purdue Pharma promoted OxyContin heavily through a variety of other channels such as sponsoring pain-related educational programs and conferences, ${ }^{5}$ distributing coupons and gifts, ${ }^{6}$ and advertising in medical journals. These marketing efforts contributed to OxyContin's blockbuster success. Revenue from OxyContin sales skyrocketed from \$48 million in 1996 to \$1.1 billion in 2000 (Van Zee, 2009) and \$3.1 billion in 2010 (IMS, 2011).

Despite the marketing claims, concerns about widespread abuse of OxyContin grew as quickly as its sales. Users of the drug quickly learned that they could defeat OxyContin's controlled-release delivery system by crushing or dissolving the pill, allowing them to access the entire store of oxycodone all at once. Some of the earliest reports of OxyContin abuse and diversion occurred in Appalachia and rural areas. However, by 2001, the DEA Administrator reported that abuse had also moved to urban areas, especially Boston and Philadelphia. ${ }^{7}$ OxyContin became one of the leading prescription drugs of abuse in the U.S., surpassing all other forms of oxycodone and hydrocodone combined (Cicero et al., 2005). The aggressive marketing of OxyContin eventually concerned local and state governments, leading to a series of lawsuits. In 2007, Purdue Pharma agreed to pay over \$600 million in fines because of misleading advertising that minimized the risks of OxyContin.

\footnotetext{
${ }^{4}$ In 1996, Purdue Pharma employed 318 sales representatives themselves and contracted with an additional 300 through a co-promotion deal with Abbott Laboratories. This number increased to 1,067 in 2002 (GAO, 2003).

${ }^{5}$ Purdue Pharma funded more than 20,000 pain-related educational programs from 1996-2002 (GAO, 2003). They also provided significant amounts of funding to several medical societies such as the American Pain Society and JCAHO (https://ag.ny.gov/sites/default/files/oag_opioid_lawsuit.pdf), organizations which recommended more aggressive diagnosis and treatment of pain.

${ }^{6}$ As noted in the GAO report (2003), "according to DEA, Purdue's use of branded promotional items to market OxyContin was unprecedented among schedule II opioids, and was an indicator of Purdue's aggressive and inappropriate marketing of OxyContin."

7 See DEA Administrator Asa Hutchinson's Testimony on December 11, 2001: https://www.govinfo.gov/content/pkg/CHRG-107hhrg77734/html/CHRG-107hhrg77734.htm, last accessed November 4, 2019.
} 


\subsection{Identifying Variation in Exposure to OxyContin}

This study exploits previously unexplored geographic variation in OxyContin's initial marketing and supply. To understand how OxyContin was marketed, we made Freedom of Information Act (FOIA) requests to obtain recently unsealed documents in Florida, ${ }^{8}$ Washington, ${ }^{9}$ and West Virginia ${ }^{10}$ from settled court cases and investigations involving Purdue Pharma in these states. These documents provide a rare look at a pharmaceutical firm's detailed marketing strategies. Among these documents, we obtained the official launch plan for OxyContin, the focus group research conducted prior to the launch, and annual itemized budgets for OxyContin from 1996-2002. Examples of these records are shown in Appendix Figure A1. We combined this information with court filings available online from Massachusetts. ${ }^{11}$

These documents reveal that Purdue Pharma would have difficulty penetrating markets that had enacted a state policy known as a "triplicate prescription program" and suggested that it would target less marketing to these states.

\subsubsection{What are Triplicate Prescription Programs?}

Triplicate prescription programs were among the earliest prescription drug monitoring programs enacted to reduce the diversion and misuse of controlled substances. Triplicate programs mandated that doctors use state-issued triplicate prescription forms when prescribing Schedule II controlled substances (which includes many opioids). The physician was required to maintain one copy of the triplicate form for their records. The patient was given two copies to give to the pharmacy; the pharmacy kept one and sent the third copy to the state drug monitoring agency. The state agency maintained a database from these forms to monitor and investigate prescribing irregularities and diversion.

Triplicate programs were adopted decades before OxyContin's launch. California adopted the first triplicate program in 1939 (Joranson et al., 2002) due to concerns of the growing diversion of opium-based pharmaceuticals (Simoni-Wastila and Toler, n.d.). California was also the last state in the country with a triplicate program, ending the program in 2004. They,

\footnotetext{
${ }^{8}$ In November 2001, the Florida Attorney General opened an investigation into Purdue Pharma's marketing tactics. The investigation was closed about a year later. Purdue Pharma offered the state of Florida a settlement of \$2 million for development of an electronic prescription monitoring program.

${ }^{9}$ State of Washington v. Purdue Pharma L.P. et al. (filed September 2017)

${ }^{10}$ State of West Virginia v. Purdue Pharma et al. (filed June 11, 2001, settled in 2004)

${ }^{11} \mathrm{https}$ ://www.documentcloud.org/documents/5715954-Massachusetts-AGO-Amended-Complaint-2019-01-

$\underline{31 . h t m l}$, last accessed July 22, 2019
} 
like other states, adopted an electronic system to work in tandem with the triplicate prescription forms before eventually eliminating the triplicate requirement. Several other states followed California's model including Idaho, Illinois, Indiana, Michigan, New York, and Texas, ${ }^{12}$ adopting triplicate programs between 1961 through 1988. ${ }^{13}$ Indiana and Michigan ended their triplicate programs shortly before OxyContin's launch. ${ }^{14}$

The academic literature on triplicate programs has found that these programs led to dramatic reductions in the prescribing of drugs subject to the policy (Simoni-Wastila et al., 2004; Hartzema et al., 1992; Weintraub et al., 1991; Sigler et al., 1984). ${ }^{15}$ There are two main reasons why triplicate programs could deter OxyContin prescribing. First, physicians in triplicate states were concerned about government oversight of their prescribing behavior (Berina et al., 1985). As Purdue Pharma observed in their focus group research: "The triplicate laws seem to have a dramatic effect on the product usage behavior of the physicians.... The doctors did not want to provide the Government with any ammunition to question their medical protocols relative to pain management. The mere thought of the government questioning their judgement created a high level of anxiety" (Groups Plus, 1995, p. 24). Although electronic monitoring programs also involved government oversight, relative to electronic systems, "It was felt that paper forms, tangible reminders of such scrutiny when handled by the prescribing physician and dispensing pharmacist, would have a greater effect on reduced prescribing and dispensing than would an

\footnotetext{
${ }^{12}$ In addition, Washington adopted a triplicate program but due to limited funding, triplicate forms were required only for physicians disciplined for drug-related violations (Simoni-Wastila and Tompkins, 2001; Fishman et al., 2004).

${ }^{13}$ Idaho adopted its program in 1967, switching to a duplicate program in 1997 (Joranson et al., 2002; Fishman et al., 2004, see also: https://legislature.idaho.gov/wp-content/uploads/OPE/Reports/r9901.pdf). Illinois enacted its triplicate program in 1961, ending in 2000 when it was replaced by an electronic system (see footnote 85 of https:/www.isms.org/opioidplan/). New York enacted a triplicate program in 1972 (Joranson et al., 2002), which ended in 2001 (NY Bureau of Narcotic Enforcement, personal communication, May 3, 2019). Texas adopted a triplicate system in 1982 (Sigler, 1984), converting to an electronic system in 1999 (see https://www.pharmacy.texas.gov/DPS.asp).

${ }^{14}$ Indiana's triplicate program began in 1987, but it was replaced by an electronic and single-copy program in 1994 (Joranson et al., 2002). Michigan enacted a triplicate program in 1988, but it also ended in 1994 (Joranson et al., 2002, see also: https://www.legislature.mi.gov/documents/2001-2002/billanalysis/Senate/htm/2001-SFA-0660E.htm).

${ }^{15}$ One study of an academic teaching hospital in Texas found that there was an immediate $60.4 \%$ reduction in prescribing of Schedule II drugs for outpatients after the state adopted its triplicate program in 1982; prescribing of non-Schedule II drugs increased over the same time period (Sigler et al, 1984). Another study examined New York's unique inclusion of benzodiazepines (a schedule IV drug) in the triplicate program starting in 1989. The study found that prescribing of these drugs declined in New York (by 30-60\% depending on the insurance type), while prescribing remained stable in a set of control states (Weintraub et al., 1991).
} 
electronic system that remained largely invisible to health care practitioners" (Simoni-Wastila and Toler, n.d.).

Second, the hassle costs to the physician of triplicate programs were especially large. According to Purdue Pharma's research: "Writing triplicate prescriptions was more trouble than others, due to the details of the forms and the various people that need to be copied to them. To the extent that they [physicians] can avoid this extra effort, they will try to follow alternative protocols" (Groups Plus, 1995, p. 24). Placing this burden specifically on the prescriber rather than on the pharmacist suggests a key reason for why triplicate programs are found in the literature to have substantial effects on prescriptions while some modern electronic prescription drug monitoring programs (particularly, non-mandate PDMPs) have been shown to have more muted effects (Buchmueller and Carey, 2018). Also, triplicate programs required the prescriber to store their copy of the prescription for a number of years, an additional cost unique to triplicate programs. In contrast, other paper-based systems did not require physicians to keep a copy, reducing the hassle cost and salience of those programs.

\subsubsection{Purdue Pharma's Views on Triplicate States}

"Triplicate states" are mentioned repeatedly in Purdue Pharma's internal documents, but there is never any mention of other existing state policies such as electronic, duplicate, or singlecopy monitoring programs. Their concern that the triplicate programs were an especially important barrier to OxyContin prescribing is founded on the information it obtained when researching the market for OxyContin. Purdue Pharma's focus group research emphasized that physicians in triplicate states would be less willing to prescribe OxyContin because of its Schedule II designation. ${ }^{16}$ "The PCPs [primary care physicians] and surgeons in the nontriplicate state (New Jersey) indicated a very high likelihood of using OxyContin for selective treatment of non-cancer related pain, and the rheumatologists in Connecticut also felt it had a place in their practice" (Groups Plus, 1995, p. 39). In contrast, the response from physicians in triplicate states was quite negative. The same report notes that "The physicians in the triplicate

\footnotetext{
${ }^{16}$ There were two separate focus group analyses. In one, physicians from New Jersey, Connecticut, and Texas were surveyed (Groups Plus, 1995). In the other, physicians attending the American College of Osteopathic Family Physicians meeting in Orlando were surveyed (Strategic Business Research, 1996). In the results from this latter research study, physicians are divided into whether they practiced in triplicate or non-triplicate states and most of the results are stratified by triplicate status, suggesting the importance of this designation to Purdue Pharma.
} 
state did not respond positively to the drug, since it is a Class II narcotic which would require triplicate prescriptions. Therefore, only a few would ever use the product, and for them it would be on a very infrequent basis" (Groups Plus, 1995, p. 36). The lack of enthusiasm for OxyContin by doctors in triplicate states is repeated dozens of times throughout these documents. ${ }^{17}$

Based on this research, Purdue Pharma's launch plan acknowledges that "these regulations create a barrier when positioning OxyContin" (Purdue Pharma OxyContin Launch Plan, 1995, p. 4). Additionally, the focus group study concludes that while "there seems to be a definite opportunity for OxyContin as a medication for treatment of severe non-cancer pain among doctors in non-triplicate states. More work might have to be done to determine if the product is viable in the triplicate states; however, the preliminary evidence is not encouraging" (Groups Plus, 1995, p. 4). Since there would be lower returns to promoting OxyContin in triplicate states, they recommended that "the product [OxyContin] should only be positioned to physicians in non-triplicate states" (Groups Plus, 1995, p. 55). Further they noted that "our research suggests the absolute number of prescriptions they [physicians in triplicate states] would write each year is very small, and probably would not be sufficient to justify any separate marketing effort" (Groups Plus, 1995, p. 49).

Purdue Pharma appears to have also lobbied for the repeal of triplicate state policies. For example, the 1999 budget plan includes a \$750,000 line-item to fund a "Program to impact the regulatory environment for opioid prescribing in triplicate states" (Purdue Pharma Budget Plan,

\footnotetext{
${ }^{17}$ In a few other representative examples from the focus group research: "The impact of the triplicate laws was particularly significant when one realizes that the most common narcotic used by the surgeons and PCP's in New Jersey [a non-triplicate state] was Percocet/Percodan, whereas in Texas [a triplicate state], this was a product/class of drugs prescribed by most doctors less than five times per year...if at all" (Groups Plus, 1996, p. 24) and "the overall reactions to OxyContin were very mixed. The most positive were the PCP's and surgeons in New Jersey [a non-triplicate state] who viewed this to be an important innovation relative to the treatment of noncancer pain, and definitely would incorporate the product into their medication protocols." (Groups Plus, 1995, p. 36) "The doctors in the triplicate states were not enthusiastic about the product at all, with only a couple indicating they would ever use it, and then in very infrequent situations." (Groups Plus, 1995, p. 39). Also, "Targeting will be a key element to the success of OxyContin. Identification of the family practitioners treating hospice patients as well as patients with moderately severe to severe injury/trauma and post-op pain will lead to faster adoption and use. Unfortunately, physicians in triplicate states are going to be harder to convince since they use less CII [schedule II] medications" (Strategic Business Research, 1996, p. 7) and "These triplicate state physicians are far less likely to use an oxycodone product to treat this level of pain. Only $14 \%$ mentioned the use of oxycodone products for moderately severe pain, whereas almost three times this number of the non-triplicate physicians (37\%) utilize this class of opioid.” (Strategic Business Research, 1996, p. 13)
} 
1999, pg. 68). In the following year's budget plan, they again included $\$ 750,000$ to fund a "Regulatory Environment Program," which may have also been related to triplicate programs. ${ }^{18}$

The statements made in these internal documents suggest that Purdue Pharma viewed triplicate programs as a substantial barrier to OxyContin prescribing and would initially target less marketing to triplicate states because of the lower expected returns. While we do not have data that breaks down Purdue Pharma's initial marketing spending by state to confirm this directly, we will show that the triplicate states had among the lowest OxyContin adoption rates in the country.

\subsubsection{Classifying Triplicate States at the Time of OxyContin's Launch}

Our empirical strategy compares OxyContin prescribing and fatal overdose trends in triplicate and non-triplicate states. We consider the non-triplicate states to be more exposed to OxyContin's introduction because the barriers to prescribing were lower and there appears to have been was more initial marketing targeted to these states. We define "triplicate states" as the five states with active triplicate programs at the time of OxyContin's launch in 1996: California, Idaho, Illinois, New York, and Texas. ${ }^{19}$ All other states are classified as "non-triplicate states." The enactment and end years of the triplicate programs are listed in the top row of Table 1. The triplicate programs were adopted decades before the opioid epidemic began, not in response to the increased prescription opioid use and abuse that began in the mid-1990s.

\footnotetext{
${ }^{18}$ There are also earlier mentions of triplicate programs beginning with the launch plan in 1996 which earmarked $\$ 200,000$ to fund a "Triplicate States Congress" and the 1998 budget plan earmarked \$150,000 for "Opioid Prescribing Regulatory Guidelines CME program," which is described as providing "physicians with an understanding of improving trends in regulation of opioid use in non-cancer pain and how to effectively prescribe within those regulations."

${ }^{19}$ In one instance in the internal documents that we reviewed, there is an incorrect reference to "nine triplicate states" when discussing retail pharmacy distribution. Since this statement was factually inaccurate at the time it was written, we cannot be certain which set of states were being referenced. It is possible that they were referring to the nine states with paper-based monitoring systems (including duplicate and single-copy programs) at that time, since this statement appears in the context of pharmacists" concerns about the "voluminous paperwork" required in these states, which would be a consideration with any paper-based system. To the degree that Purdue Pharma was also concerned about other paper-based programs (although these were never mentioned elsewhere in the documents) and also marketed less in these states, our results will be attenuated. That said, triplicate programs and the burdens for the physician (not the pharmacy) are always the focus of the discussion in the internal documents we obtained. These documents specifically mention the hassle of triplicate programs to prescribers due to the "various people that need to be copied to them" which would apply only to states with active triplicate programs and not other paperbased programs. This suggests that the discussions of the triplicate states referenced throughout this paper were referring to triplicate programs specifically and not a broader set of paper-based programs.
} 
Although triplicate programs were eventually discontinued, our coding of triplicate states is irrespective of later changes. We hold each state's initial triplicate status in 1996 as fixed over the entire study period. It is unclear whether and how quickly Purdue Pharma responded to states transitioning away from triplicate programs. ${ }^{20}$ Idaho's program ended in 1997, shortly after OxyContin's introduction. The other triplicate states all ended their programs by 2004, replacing them with electronic programs. Therefore, our results will speak to the long-run effects of the initial targeting of Purdue Pharma's marketing and barriers to prescribing induced by triplicate status during the launch.

Two other states (Indiana and Michigan) had triplicate programs which were discontinued in 1994. Purdue Pharma's primary concerns with the monitoring and hassles associated with triplicate programs would no longer be present in these states at the time of the launch in 1996. While we cannot be certain in knowing exactly how Purdue Pharma treated the former-triplicate states since we do not observe the full marketing strategy, to the extent that they also received less marketing, our results will be attenuated. We analyze these states separately in Section 6. Also, it is notable that, with the exception of Idaho, the triplicate states are among the largest states in the country and also have some of the largest urban centers. In robustness tests, we will consider the possible role of these distinct characteristics with special attention to population size and urbanicity.

\section{$3 \quad$ Data}

Our analysis uses data from several sources. We use data on drug overdose deaths and multiple measures of opioid distribution, prescribing, and misuse.

\subsection{Mortality Data}

We use a restricted-use version of the National Vital Statistics System (NVSS) Multiple Cause of Death mortality files from 1983 to 2017 that contains state and county of residence identifiers. ${ }^{21}$ These data represent a census of deaths in the U.S. We follow the coding used by the Centers for Disease Control (CDC) to categorize deaths as drug and opiate-related. The

\footnotetext{
${ }^{20}$ In supplementary analyses, we study state responses to triplicate program discontinuation.

${ }^{21}$ We begin in 1983 because the 1981 and 1982 files do not include all deaths. In select states, only half of deaths were included and they were included twice. This feature is not necessarily problematic for our purposes, but we chose to start our sample with the 1983 data given that this already provides a lengthy pre-period.
} 
1983-1998 data use ICD-9 codes to categorize causes of deaths while the 1999-2017 data use ICD-10 codes. ${ }^{22}$ The CDC reports that the transition from ICD-9 to ICD-10 resulted in a small increase in poisoning-related deaths (not necessarily drug poisonings) of $2 \%$ (Warner et al., 2011). Our time fixed effects help account for this transition given that we would not expect systematically different effects of the coding change across states. We explore this coding change in Appendix Figure A2 by examining the national trend in drug overdose deaths around 1999. While we observe an increase in the total drug overdose death rate in 1999, it is comparable to increases in other time periods when there were no coding changes. The 1999 increase is larger for opioid-related overdose deaths but, again, not uniquely large relative to other annual changes. Notably, the main estimates are not driven by a large differential overdose increase in 1999, suggesting that the time fixed effects are appropriately accounting for the switch to ICD-10 codes.

Given concerns over missing opioid designations on death certificates for drug-related overdoses (e.g., Ruhm, 2018), we favor using a broader measure of total drug overdose deaths which should be robust to substance-specific classification errors (Venkataramani and Chatterjee, 2019). However, we also present complementary results for opioid-related overdose deaths.

We also study disaggregated measures of drug overdose deaths by the type of opioid when available. Deaths with code T40.1 indicate poisoning by heroin, T40.2 indicate natural and semisynthetic opioids (e.g., OxyContin), and T40.4 indicate synthetic opioids excluding methadone (e.g., fentanyl). It is difficult to link deaths for specific drugs across the entire time period given differences in ICD-9 and ICD-10 codes, so we will only study overdose deaths by opioid type for 1999-2017 while highlighting the caveat that this analysis does not include the pre-OxyContin period.

\footnotetext{
${ }^{22}$ For 1983-1998, we define drug poisonings as deaths involving underlying cause of death ICD-9 codes E850E858, E950.0-E950.5, E962.0, or E980.0-E980.5 (see Table 2 of https://www.cdc.gov/drugoverdose/pdf/pdo_guide_to icd-9-cm_and_icd-10_codes-a.pdf, last accessed November 29,2018 .). When we study opioid-related overdoses, we will use deaths involving E850.0, E850.1, E850.2, or N965.0 (Alexander et al., 2018; Green et al., 2017). For the 1999-2017 data, we code deaths as drug overdoses using the ICD-10 external cause of injury codes X40-X44, X60-64, X85, or Y10-Y14 (Warner et al., 2011). We use drug identification codes, which provide information about the substances found in the body at death, to specify opioidrelated overdoses: T40.0-T40.4 and T40.6. Linking opioid overdoses across ICD-9 and ICD-10 codes in this manner is recommended in Table 3 of https://www.cdc.gov/drugoverdose/pdf/pdo guide to icd-9-cm and icd-10 codesa.pdf. One exception is our use of T40.6. The inclusion of this code does not change our results as we will show in the Appendix.
} 


\subsection{Opioid Distribution, Prescriptions, and Misuse}

We use state-level data on the legal supply of opioids from the Drug Enforcement Agency's (DEA) Automation of Reports and Consolidated Orders System (ARCOS). The Controlled Substance Act of 1970 requires all manufacturers and distributors to report their transactions and deliveries of all Schedules I and II substances as well as a number of Schedule III-V substances to the Attorney General. ARCOS is the system that monitors and records the flows of these controlled substances as they move from manufacturers to retail distributors. In the public data, only active ingredients are reported so we observe the distribution of oxycodone by state, but not OxyContin specifically. These data are available online for $2000-2017,{ }^{23}$ and we were able to collect earlier data for 1997-1999 using the WayBack Machine. ${ }^{24}$ Because of this paper's specific interest in OxyContin, we made a FOIA request for OxyContin distribution specifically and received these data for 2000-2016. ${ }^{25}$ We report all ARCOS measures in morphine equivalent doses, equal to 60 morphine milligram equivalents.

We also study measures of OxyContin prescriptions. It should be noted that prescription data from the 1990s and early 2000s are scarce at the state level. ${ }^{26}$ We use Medicaid State Drug Utilization Data (SDUD) for 1996-2005, ${ }^{27}$ which reports the number of prescriptions of outpatient drugs paid for by Medicaid agencies by National Drug Code (NDC), quarter, and state. ${ }^{28}$ While the Medicaid population is non-representative, prescriptions among this group are a potentially useful proxy for state prescribing behavior while also representing an important population disproportionately affected by the opioid crisis (e.g., CDC, 2009; Sharp and Melnik, 2015; Whitmire and Adams, 2010; Fernandes et al., 2015). Opioid Medicaid prescriptions are highly-correlated with the opioid supply measures in the ARCOS data. Annual Medicaid

\footnotetext{
${ }^{23}$ The data are found here: https://www.deadiversion.usdoj.gov/arcos/retail drug summary/, last accessed November 30, 2018.

${ }^{24}$ https://web.archive.org/web/20030220041015/https://www.deadiversion.usdoj.gov/arcos/retail_drug_summary/

${ }^{25}$ Our request for pre-2000 OxyContin data was denied; we were told that these years of data are unavailable.

${ }^{26}$ Many of the data aggregators that researchers often use to obtain prescription drug claims data (such as IQVIA) no longer maintain state-specific records for the 1990s or early 2000s.

${ }^{27}$ We end the sample in 2005 due to the introduction of Medicare Part D.

${ }^{28} \mathrm{We}$ select on state-years reporting in all four quarters (over $94 \%$ of state-years). Additionally, while a recent version of SDUD suppresses the number of prescriptions for a given NDC-state-quarter when that number is smaller than 10 , we rely on an earlier version of the data that is unsuppressed.
} 
OxyContin prescriptions per 1,000 beneficiaries $^{29}$ and per capita OxyContin supply (ARCOS) in 2000 - the first year available - have a correlation coefficient of 0.66 .

We also use a restricted version of the Medical Panel Expenditure Survey (MEPS) with state-identifiers, accessed through the AHRQ Data Facility. The MEPS is a nationallyrepresentative survey of households, including medical and pharmaceutical claims. We constructed per capita OxyContin prescriptions for 1996-2016. ${ }^{30}$ Per capita OxyContin prescriptions in the 2000 MEPS have a cross-sectional correlation with the 2000 ARCOS of 0.53 .

Finally, we study self-reported rates of opioid misuse in the past year for both OxyContin and all other pain relievers (excluding OxyContin) using the National Study of Drug Use and Health (NSDUH) for 2004-2012. ${ }^{31}$ The measure of OxyContin misuse is first available in 2004. The NSDUH is a nationally-representative household survey of individuals ages 12 and older and is the largest annual survey collecting information on substance use in the U.S. State-level metrics are publicly reported in two-year waves. ${ }^{32}$ Alpert et al. (2018) showed a strong correlation between OxyContin misuse and supply measures in the ARCOS data.

\subsection{Summary Statistics}

In Table 1, we present summary statistics for 1991-1995, representing the pre-OxyContin period, separately for each triplicate state as well as aggregated means by triplicate status. As shown previously, drug overdose death rates are higher on average in the triplicate states before OxyContin's introduction, including deaths involving opioids. With the exception of Idaho, each triplicate state had an opioid-related death rate above the median. Some of these differences can be explained by disproportionately higher rates of cocaine-related deaths in triplicate states. When overdoses involving cocaine are eliminated, the differences between triplicate and non-

\footnotetext{
${ }^{29}$ We scaled the number of prescriptions by the number of Medicaid beneficiaries using data from the University of Kentucky Center for Poverty Research (University of Kentucky Center for Poverty Research, 2018).

${ }^{30}$ We identify OxyContin prescriptions in the MEPS using NDC codes matched to First Databank as well as information in the reported prescription drug name field in the MEPS.

${ }^{31}$ The NSDUH uses techniques designed to elicit accurate and honest answers from respondents. As one example, the respondent is shown cards with the names of different types of pain relievers (including OxyContin) and photos of the pills. They are asked to identify "which of the pain relievers... have you used when they were not prescribed for you or that you took only for the experience or feeling they caused?" These methods reduce concerns that the "OxyContin misuse" measure reflects misuse of other types of oxycodone.

${ }^{32}$ For more information on these data, see Section II.A of Alpert et al. (2018).
} 
triplicate states shrink. With respect to demographic characteristics, ${ }^{33}$ triplicate states on average have larger populations and a much larger share of the population is Hispanic. The age distribution and educational attainment are similar across triplicate and non-triplicate states.

\section{Empirical Strategy}

To estimate the impact of OxyContin's introduction, we use a difference-in-differences framework comparing outcomes in non-triplicate states relative to triplicate states before and after the launch of OxyContin. We rely primarily on event-study models due to their transparency and because the timing of the effect is of interest. We report the differential change in overdose death rates for non-triplicate states relative to triplicate states given that nontriplicate states were more "exposed" to the introduction of OxyContin. The event study specification is:

$$
y_{s t}=\alpha_{s}+\gamma_{t}+\sum_{t=198}^{2017} \beta_{t} \times 1(\text { Non-Triplicate })_{s} \times 1(\text { Year }=t)+\varepsilon_{s t},
$$

where $\mathrm{y}_{\mathrm{st}}$ represents annual drug overdose deaths per 100,000 people in state $s$ in year $t$. This specification includes state $\left(\alpha_{s}\right)$ and year $\left(\gamma_{t}\right)$ fixed effects. 1 (Non-Triplicate) $)_{s}$ is an indicator based on the initial triplicate status of the state in 1996 and is fixed over the entire time period. This is interacted with a full set of year fixed effects. We present the estimates of $\beta_{t}$ along with $95 \%$ confidence intervals graphically. We normalize the $\beta_{t}$ coefficient to equal zero in 1995 , the year before OxyContin was introduced. Our main results are population-weighted, but we also show unweighted regression results in the Appendix.

We also present difference-in-differences estimates using more aggregated time intervals for the purpose of quantifying the event study results. The specification is:

$$
\begin{aligned}
y_{s t}=\alpha_{s}+\gamma_{t} & +\delta_{1} \times 1(\text { Non-Triplicate })_{s} \times 1(1996 \leq \text { Year } \leq 2000) \\
& +\delta_{2} \times 1(\text { Non-Triplicate })_{s} \times 1(2001 \leq \text { Year } \leq 2010) \\
& +\delta_{3} \times 1(\text { Non-Triplicate })_{s} \times 1(2011 \leq \text { Year } \leq 2017)+\boldsymbol{X}_{s t}^{\prime} \theta+\varepsilon_{s t} .
\end{aligned}
$$

\footnotetext{
${ }^{33}$ Demographic and population information are calculated from Current Population Study (CPS) data (Ruggles et al., 2018) and the Surveillance, Epidemiology, and End Results Program (SEER).
} 
The excluded category is 1991-1995 as we limit the sample to 1991-2017 for the difference-in-differences analyses. ${ }^{34}$ We estimate three separate "post" effects to permit some heterogeneity while still providing more aggregated effects. The first post-OxyContin time period is 1996-2000. This period represents the introduction of OxyContin, the launch of different dosages, and the initial ramp up of marketing by Purdue Pharma. We also estimate a separate effect for 2001-2010, corresponding to the "first wave" of the opioid crisis when most opioid-related deaths are attributed to prescription opioids. Finally, we estimate a separate effect for 2011-2017, representing the second and third waves of the opioid crisis when deaths from heroin and illicitly-manufactured fentanyl became more prominent.

Our controls $\left(\boldsymbol{X}_{s t}\right)$ include the fraction of the population that is white non-Hispanic, black non-Hispanic, Hispanic, the fraction ages 25-44, 45-64, 65+, the fraction with a college degree, and $\log$ population. ${ }^{35}$ We do not include some of the typical controls often included in models of opioid overdoses, including policy variables (e.g., PDMPs, pill mill laws, etc.) and economic conditions. A motivation of this paper is to understand the initial conditions of the opioid crisis, which has potentially affected a wide range of outcomes. We remain agnostic about the breadth of effects and choose not to control for these types of covariates in our main specification given that these covariates may also be outcomes. However, we will show that our results are robust to conditioning on subsequent policy adoption and economic conditions.

In addition, some of our outcome variables are only available after 1996, such as OxyContin supply. Despite the lack of a pre-period, it will be useful to analyze cross-sectional differences between triplicate and non-triplicate states. For these select outcomes, we estimate the non-normalized mean differences in each time period as follows:

$$
\begin{aligned}
y_{s t}=\gamma_{t} & +\delta_{1} \times 1(\text { Non-Triplicate })_{s} \times 1(1996 \leq \text { Year } \leq 2000) \\
& +\delta_{2} \times 1(\text { Non-Triplicate })_{s} \times 1(2001 \leq \text { Year } \leq 2010) \\
& +\delta_{3} \times 1(\text { Non-Triplicate })_{s} \times 1(2011 \leq \text { Year } \leq 2017)+\boldsymbol{X}_{s t}^{\prime} \theta+\varepsilon_{s t}
\end{aligned}
$$

\footnotetext{
${ }^{34}$ For the difference-in-differences specification, we condensed the pre-period to 5-years (from the full 13 years available) to provide a more meaningful comparison with the post-periods. As can be seen in the event-study results, the estimates are not sensitive to different choices for the pre-period.

${ }^{35}$ In the Appendix, we show robustness tests that interact a set of these covariates with year indicators, permitting them to have differential effects in each year.
} 
We will also show how the trajectories of these outcomes differ between triplicate and nontriplicates more flexibly by interacting the non-triplicate indicator in the above specification with year fixed effects. We present the coefficients on these interaction terms graphically.

Because we have a small number of (un)treated states, traditional cluster covariance estimators may produce standard error estimates that are too small (Conley and Taber, 2011). For this reason, we use a restricted wild cluster bootstrap method at the state level to account for serial correlation in all models. ${ }^{36}$ We use a 6-point weight distribution as suggested by Webb (2014) which provides more reliable inference than the typical 2-point Rademacher weights when there are few clusters. Webb (2014) points out that using Rademacher weights when there are a small number of clusters will produce too few unique bootstrap samples and t-statistics to generate meaningful p-values. In a difference-in-differences framework, a related problem occurs when there are a very small number of treated or untreated clusters (Brewer et al., 2018). ${ }^{37}$ The 6-point distribution improves the reliability of the wild bootstrap in both scenarios. Given p-values for a range of null hypotheses, we construct and report $95 \%$ confidence intervals, which will not be symmetric using this approach. ${ }^{38}$ In the Appendix, we show that traditional "clustered" standard errors produce tighter confidence intervals than the restricted wild bootstrap method. We also show that permutation tests produce similar results.

\section{$5 \quad$ Results}

Our analysis begins by documenting the large differences in OxyContin exposure across triplicate and non-triplicate states. We then estimate the impact of these differences on drug overdose deaths over the short and long run. We also investigate alternative explanations for these patterns including trends in the distribution and misuse of other opioid drugs, differential policy adoption, and economic shocks. We explore mechanisms for the long-run mortality effects in the next section.

\footnotetext{
${ }^{36}$ Specifically, we use a restricted wild bootstrap in which a null hypothesis is imposed and then a $t$-statistic is compared to distribution of placebo $t$-statistics (this is method 13 on page 418 of Cameron et al. 2008).

${ }^{37}$ In this case, the bootstrapped t-statistics are all within the neighborhood of a finite number of values so there is limited independent variation.

${ }^{38}$ We use the boottest package in Stata (Roodman et al., 2018) to implement this procedure.
} 


\subsection{Effects of Triplicate Status on OxyContin Exposure}

We first show that non-triplicate states were more exposed to the introduction of OxyContin as measured by OxyContin distribution and prescriptions per capita. The raw trends for these outcomes are presented in Figure 3 using three different data sources. Panel A presents trends in the distribution of OxyContin measured in morphine equivalent doses (MEDs) per capita using ARCOS data. These data represent a virtual census of OxyContin distribution in the U.S. In 2000, there is over two and a half times more OxyContin distribution per capita in nontriplicate states compared to triplicate states. In the first two columns of Table 2, we present estimates of equation (3) for this outcome with and without time-varying controls. The differences in OxyContin distribution across triplicate and non-triplicate states are quantitatively large and statistically significant in all time periods and these differences persist through 2016.

A limitation of the ARCOS data is that it is only available back to 2000. As complementary measures of OxyContin exposure, we study two other data sources that enable us to observe OxyContin prescriptions for earlier years. Panel B of Figure 3 shows trends for Medicaid OxyContin prescriptions per 1,000 beneficiaries from 1996-2005. Panel C shows OxyContin prescriptions per 1,000 people using the restricted-access MEPS for 1996-2016, which is noisier due to its small sample size. In both datasets, we observe much higher rates of OxyContin prescriptions in non-triplicate states. These differences are apparent as early as 1996 in the Medicaid data and 1997 in MEPS. ${ }^{39}$ OxyContin prescribing increases rapidly during the first several years after its launch; however, there is a striking reduction in total OxyContin prescriptions and distribution in 2005-2006, revealing some important dynamics in early OxyContin sales and promotion. ${ }^{40}$ OxyContin prescribing decreases again after Purdue Pharma

\footnotetext{
${ }^{39}$ There are no OxyContin prescriptions in the 1996 MEPS. The 1996 MEPS has the smallest number of individuals, households, and prescriptions of all the MEPS samples. This reduced size combined with the limited national exposure to OxyContin in 1996 is consistent with not finding any OxyContin prescriptions in the 1996 data. ${ }^{40}$ One possible explanation for this decline (which is apparent in both the ARCOS and MEPS data) is the end of a copromotion agreement with Abbott Laboratories. Abbott provided at least 300 sales representatives through this agreement to sell OxyContin (GAO, 2003), initialing doubling Purdue Pharma's sales force, and a company executive documented in 1997 that $25 \%$ of OxyContin prescription were written by “Abbott MD's." This agreement with Abbott ended around the time that we see the dramatic drop in OxyContin distribution (https://www.statnews.com/2016/09/22/abbott-oxycontin-crusade/, last accessed May 8, 2019). Abbott decided not to renew this relationship given early reports about OxyContin abuse and the federal government's concerns over these reports. By 2008, Purdue Pharma began to increase its sales force again (p. 72 in Commonwealth of Massachusetts, 2018). Additionally, Purdue Pharma was under investigation for 5 years before its 2007 settlement was reached related to misleading marketing practices (https://www.judiciary.senate.gov/imo/media/doc/Brownlee\%20Testimony\%20073107.pdf). This investigation may have also affected the company's marketing practices and sales over this time period.
} 
released an abuse-deterrent version in 2010. However, non-triplicate states continue to experience differential exposure to OxyContin throughout these downturns.

Finally, we examine how the initial "adoption" of OxyContin varied across triplicate and non-triplicate states. Panel A of Appendix Figure A3 uses Medicaid data to show the number of OxyContin prescriptions per 1,000 beneficiaries in 1996 for states reporting data in 1996. The triplicate states (highlighted in red) largely cluster near the bottom of the distribution. Four of the triplicate states (CA, IL, NY, TX) are among the five states with the lowest number of OxyContin prescriptions per capita in 1996, although Idaho is an exception with higher prescribing. ${ }^{41}$ While we cannot report state-specific figures from the restricted-use MEPS, Panel $B$ uses the first available year of ARCOS data. The pattern is similar in ARCOS with four of the triplicate states positioned among the lowest seven states in the distribution (Idaho is ranked $14^{\text {th }}$ ). These results show that triplicate states initially had some of the lowest rates of OxyContin adoption in the country.

\subsection{Effects of Triplicate Status on Exposure to Other Opioids}

We next examine whether there are differences in the use of other prescription opioids across triplicate and non-triplicate states that could also contribute to differences in overdose death trends. First, we use the ARCOS data to compare trends in the distribution of oxycodone versus hydrocodone. Unlike oxycodone which is a Schedule II drug, hydrocodone (e.g., Vicodin) was classified as a Schedule III drug. ${ }^{42}$ Therefore, it would not be subject to triplicate programs which cover Schedule II drugs. Figure 4 shows trends in the distribution of these opioid drugs starting in 1997, the earliest year of data available. The trends are presented in morphine equivalent doses (MEDs) which adjust for the different potency of these drugs. Remarkably, per capita hydrocodone distribution, which should be unaffected by triplicate programs, is nearly identical in triplicate and non-triplicate states over the entire 1997-2017 time period. In triplicate states, oxycodone and hydrocodone distribution are also identical. However, there are large differences in oxycodone distribution between triplicate and non-triplicate states.

\footnotetext{
${ }^{41}$ This may reflect that Idaho was in the process of replacing its triplicate program at the time. We do not know whether Purdue Pharma anticipated this legislative change and adjusted their promotional activities in response. 42 On October 6, 2014, hydrocodone combinations were switched from Schedule III to Schedule II.
} 
The difference in oxycodone distribution between the two sets of states exceeds the difference observed for OxyContin alone in Figure 3 and grows over time; this growth suggests possible spillovers of OxyContin's promotion on the use of other oxycodone combination products (e.g., Percocet). This would be consistent with Purdue Pharma's marketing strategies that aimed to expand the opioid market for chronic pain by making doctors more comfortable with prescribing strong oxycodone products. This messaging could spill over to other oxycodone combination products.

In columns (3) and (4) of Table 2, we report the estimated differences in oxycodone distribution across triplicate and non-triplicate states using equation (3). These differences are statistically significant and growing from the initial period observed in the data to the last period. In columns (5) and (6), we do not observe statistically significant differences in hydrocodone at any point in time.

In Figure 5, we show trends in opioid misuse rates for OxyContin versus all other pain reliever misuse (excluding OxyContin) using the National Study of Drug Use and Health (NSDUH) from 2004-2012. Non-triplicate states have about twice as much OxyContin misuse as triplicate states (Panel A). However, we do not observe any meaningful differences in pain reliever misuse excluding OxyContin (Panel B). Thus, differences in opioid misuse across triplicate and non-triplicate states is unique to OxyContin.

Taken together, these results are consistent with any differences in overdose rates being primarily attributable to OxyContin, since the primary differences between triplicate and nontriplicate states are exposure to and misuse of OxyContin. The differences in OxyContin distribution and misuse continue even through the most recent years of data, consistent with long run differences in mortality rates. In Section 6, we will discuss the mechanisms for why triplicate programs have such persistent effects on OxyContin exposure.

These results also suggest that the broader changes in attitudes towards prescribing opioids or the adoption of new treatment guidelines for pain (e.g., $2001 \mathrm{JCAHO}$ revisions) that took place over this time period did not differentially affect triplicate and non-triplicate states, since we would expect these changes to also impact the prescribing of hydrocodone and other opioids. Instead, we do not observe differences in trends or even levels across triplicate and nontriplicate states in hydrocodone distribution. We also do not observe meaningful cross-sectional differences in pain reliever misuse when OxyContin is excluded. 


\subsection{Effects of OxyContin Exposure on Drug Overdose Deaths}

Next, we examine whether the differential exposure to OxyContin led to differences in drug overdose deaths over time. The raw trends in drug overdose deaths for triplicate and nontriplicate states were previously shown in Figure 2. In Figure 6, we present the coefficients from estimating the event-study specification in equation (1) with $95 \%$ confidence intervals. Panel A shows the rate of total overdose deaths as the outcome and Panel B shows the rate of opioidrelated overdose deaths. Prior to OxyContin's launch, the coefficients are close to zero and largely statistically insignificant, suggesting that there were no systematic differences in trends prior to $1996 .{ }^{43}$ However, within a few years after the launch, the trends diverge. Drug overdose deaths increase much more rapidly in non-triplicate states than in triplicate states. The coefficient estimate in 1997 indicates that overdose deaths in non-triplicate states increased by 0.25 deaths per 100,000 compared to triplicate states. These effects increase to a statistically significant 2.25 deaths per 100,000 in 2002 and 11.41 deaths per 100,000 in 2017. It is not surprising that the effects on overdose deaths are delayed given the low levels of OxyContin sales in the earliest years, expansions in promotion over time, and the FDA's relabeling in 2001 that expanded the market for chronic use. Additionally, it would take time before OxyContin users transitioned to misuse and dependence.

In Panel B of Figure 6, we show the analogous event-study results for opioid-related deaths specifically. The pattern is similar to Panel A, suggesting that the overall mortality effects are largely driven by opioids. We show results for both overdose death measures throughout the paper out of concern that opioid deaths may be under-reported. The broader total overdose death measure will capture some opioid overdose deaths that were not coded as such and provides the most consistently defined measure over our 35-year study period. The event study results are also similar without population-weights or when we condition on time-varying covariates (see Appendix Figure 4). These event-study results strongly suggest a causal relationship between the introduction of OxyContin and the rise in overdose deaths. We further examine the causality of this relationship with a battery of robustness tests in Section 5.4.

\footnotetext{
${ }^{43}$ To the extent that the general pain management culture was changing and perhaps systematically across triplicate and non-triplicate states, we would expect to observe gradual responses beginning even prior to OxyContin's launch given concerns about the under-treatment of pain in the 1980s and early 1990s. However, we do not observe preexisting trends reflecting differential responses to changing prescribing practices.
} 
To quantify the magnitude of these effects, in Table 3 , we present difference-indifferences estimates from equation (2) for the total drug overdose death rate and the opioid overdose death rate separately. In Column 1 of Table 3, we present unweighted estimates. Relative to the baseline 1991-1995 period, non-triplicate states experienced a relative annual increase in total overdose deaths of 1.244 per 100,000 people in the earliest years after the launch (1996-2000). This effect is statistically significant at the 5\% level. ${ }^{44}$ By 2011-2017, the relative increase grew to 6.248 fatal overdoses per 100,000. In Column 2, we present populationweighted estimates. The point estimates are slightly larger in magnitude. Column 3 shows that the estimates are robust to including time-varying covariates. Non-triplicates experienced a differential rise in overdoses of 1.125 per 100,000 for 1996-2000. We estimate that the "counterfactual" fatal overdose rate for non-triplicates during this time period would have been 4.312 per 100,000 if they had been triplicate states, implying that the increase in fatal overdoses represents a $26 \%$ increase. ${ }^{45}$ The estimated effect grows to 4.221 in 2001-2010, representing a $60 \%$ increase, and 6.944 by 2011-2017, representing a $62 \%$ increase for non-triplicate states. In Column 4, we include Census region-by-time interactions to account for geographic differences in overdose rate growth. The results are generally similar. The three "post" estimates are jointly significant from zero at the $1 \%$ level.

The bottom panel of Table 3 shows the same results for opioid-related overdose deaths. The patterns are similar. The 1996-2000 estimate in Column 3 implies a $62 \%$ increase for nontriplicate states; and the 2011-2017 estimate indicates that initial non-triplicate status increased opioid-related death rates by over $72 \%$. Thus, proportionally, we find larger effects for opioidrelated deaths than total overdose deaths, consistent with OxyContin exposure having a disproportionate effect on overdoses that report opioid involvement.

\footnotetext{
${ }^{44}$ If we limit our difference-in-differences analysis to 1991-1998, we still estimate a statistically significant nontriplicate effect on the overdose rate in the shorter post-period. These years of data use only ICD-9 codes, so this is reassuring that we are not estimating an artifact of the data due to a change in ICD codes. Notably, we also do not observe a large differential jump in the event study coefficients in 1999 when this switch occurred.

${ }^{45}$ The counterfactual is the overdose rate of the non-triplicate states minus the estimated coefficient on the nontriplicate indicator in that time period. Specifically, the "counterfactual" fatal overdose rate in non-triplicate states (had they been triplicate states) during this period is $4.312(=5.437-1.125)$ such that the implied percentage increase is $1.125 / 4.312=0.26$.
} 


\subsubsection{State-Specific Results}

The estimated differences between triplicate and non-triplicate states are not due to one outlier triplicate state experiencing uniquely low growth in overdose deaths. Instead, we observe this pattern for all triplicate states. In Figure 7, we analyze the rate of growth in overdose death rates before and after OxyContin's introduction comparing each triplicate state to its bordering neighbor states. Specifically, we compare the change in the overdose death rate between the ten years after OxyContin's launch (1996-2005) relative to the ten years before (1986-1995). We find that for four out of the five triplicate states, the triplicate state had the smallest growth rate relative to all of their bordering states and the one exception - Illinois - had the second-to-lowest growth rate. ${ }^{46}$ This pattern is not specific to the chosen set of years. Appendix Figure A5 repeats this exercise but uses the most recent 10 years of data (2008-2017) as the post-period. The remarkable consistency of low overdose growth across the triplicate states relative to other states in their regions strongly suggests that it was the triplicate program and not other characteristics that drove the relatively slow growth in these states, though we explore alternative explanations further below.

\subsubsection{Heroin and Fentanyl Overdose Deaths}

We next examine trends in overdose deaths by the type of opioid. Appendix Figure A6 shows cross-sectional annual differences in opioid-related overdose deaths comparing triplicate and non-triplicate states for: natural and semisynthetic opioids (e.g., oxycodone and hydrocodone), heroin, and synthetic opioids (e.g., fentanyl) for 1999-2017. ${ }^{47}$ Prior to 2010, the only meaningful difference in overdose mortality between triplicate and non-triplicate states is for natural and semisynthetic opioids, the category which includes OxyContin. Interestingly, we observe a large relative increase in heroin-related fatal overdoses in non-triplicate states starting in 2011, although the differences are not statistically significant. This is consistent with the findings in Alpert et al. (2018) and Evans et al. (2019) which showed that areas with high initial rates of OxyContin misuse or oxycodone supply experienced faster growth in heroin deaths after

\footnotetext{
${ }^{46}$ While Idaho had a higher OxyContin adoption rate than other triplicate states, many of its neighbors did too, suggesting meaningful regional differences. For Idaho, this higher rate of adoption did not translate into a high growth rate in overdoses, which might suggest a high demand for legitimate uses of the product in this state. ${ }^{47}$ The specific type of opioid involved in overdose deaths is not reliably coded before 1999 in a manner that can be linked to 1999-2017 data.
} 
an abuse-deterrent version of OxyContin was introduced in 2010. We also find that sharp differences in synthetic opioid overdose death rates emerged in 2014, which is consistent with reformulation leading to fentanyl use when it became mixed with the United States heroin supply (Ciccarone, 2017; Pardo et al., 2019).

The timing of these differential drug-specific trends suggests that the introduction of OxyContin had long-term effects on drug overdose deaths through each wave of the opioid crisis. Initially, we observe growth in overdose deaths involving prescription opioids. After OxyContin became hard to abuse and the opioid crisis transitioned first to heroin and then to fentanyl, states less exposed to OxyContin's introduction were also less affected by these transitions.

\subsubsection{Non-Fatal Outcomes}

As a measure of non-fatal harms resulting from OxyContin's introduction, in Appendix Figure A7, we examine differences in substance abuse treatment admissions for opioids using the Treatment Episode Data Set (TEDS). ${ }^{48}$ The results are consistent with the drug overdose death findings. Non-triplicate states experienced sharper growth in substance abuse treatment admissions for opioids after OxyContin's introduction. These results help corroborate the mortality findings while also providing evidence that OxyContin exposure also affected less extreme outcomes, such as substance abuse.

\subsection{Alternative Explanations}

In this section, we explore alternative explanations for our findings and test the robustness of our results. These robustness tests are presented in Table 4. Column 1 repeats the main baseline estimate (Column 3 of Table 3). We focus our discussion on total overdose deaths but find generally similar results for opioid overdose deaths (see Appendix Table A1).

\footnotetext{
48 The TEDS includes all admissions into treatment facilities receiving public funding. While TEDS is often used in substance use research, there are concerns about underreporting of admissions. Some states may not report in each year or may not report admissions in the same manner over time (SAMHSA, 2013). Our assumption is that triplicate states did not systematically change reporting behavior around 1996. We tested this assumption explicitly by replicating the analysis for other substances (e.g., marijuana, alcohol) and do not observe similar patterns, suggesting that reporting issues are not driving the results.
} 


\subsubsection{Population Size}

It is notable that four of the triplicate states are among the largest states in the country. One concern is that states with large populations and major urban cities would have experienced systematically different trends in overdose deaths independent of their triplicate status. As one test of this alternative hypothesis, we compare triplicate states to the largest non-triplicate states. In Column 2 of Table 4, we select the four largest non-triplicate states in terms of 1990 population size (FL, PA, OH, and $\mathrm{MI}$ ) as comparison states for the four largest triplicate states. We replicate our difference-in-differences analyses with these select states. ${ }^{49}$ The estimates are much larger than the main estimates which indicate that triplicate states have uniquely low overdose death rate growth even when compared to the largest non-triplicate states. Despite the additional noise due to the much smaller sample size, the estimates remain statistically different from zero. More generally, when we look across all states, we find little correlation between population size and overdose death rate growth, especially at the top of the distribution. Appendix Figure A8 shows state-level changes in 10-year growth rates sorted by population size.

A related concern is that the triplicate and non-triplicate states vary in terms of urbanicity which could also lead to systematically different overdose death trends. In Panel A of Appendix Figure A9, we replicate our event study design at the county-level for urban counties (826 counties). ${ }^{50}$ We estimate larger effects for urban counties than our main results, implying that urban counties in triplicate states have uniquely low fatal overdose growth even compared to urban counties in non-triplicate states. Within urban counties, there are still some meaningful size differences, so in Panel B, we further select counties with the largest population size: "central counties of metro areas of 1 million population or more" (175 counties). ${ }^{51}$ Again, the results are similar, meaning that even when we select on the largest metropolitan areas in the country, we observe large differences in fatal overdose rate growth based on 1996 triplicate status. While there may still remain some differences in the characteristics of these metropolitan areas across triplicate and non-triplicate states, it is notable how insensitive the main results are to selecting further on these dimensions.

\footnotetext{
${ }^{49}$ We exclude Idaho from this analysis, though results are similar if we include it.

${ }^{50}$ We use the 1993 categorization by the Office of Management and Budget which divides counties into metropolitan ("urban") and non-metropolitan ("rural").

51 This categorization is defined by the Department of Agriculture's Economic Research Service and we use the 1993 values.
} 


\subsubsection{Adoption of Other Policies}

Triplicate states were some of the earliest adopters of drug monitoring programs and so were potentially also at the frontier of reducing prescription drug abuse in the years following OxyContin's introduction. If triplicate states followed different policy paths which addressed opioid misuse more effectively than those in non-triplicate states, this could be confounding our results. In Column 3 of Table 4, we examine the drug overdose death rates in triplicate states compared to outcomes in states with other types of PDMPs in 1996-electronic PDMPs and duplicate programs. Ten states had implemented these programs by 1996 (Horwitz et al., 2018). These non-triplicate programs were not mentioned in Purdue Pharma documents, yet if we believe that some states were simply "ahead of the curve" in moderating opioid misuse, then we would expect that states with electronic PDMPs and other monitoring programs would also experience slower growth in overdose death rates. However, the estimates actually increase when we use this sample, suggesting that triplicate states experienced uniquely small changes in overdose growth even relative to states with other types of PDMPs. ${ }^{52}$

As a complementary approach, in Column 4 of Table 4, we replicate the difference-indifferences analysis for the full sample of states while controlling for a set of opioid-related policy variables. We include two indicators for PDMPs from Horwitz et al. (2018) concerning the enactment of a PDMP and enactment of a modern, electronic system. In addition, we also include indicators for the adoption of "must access" PDMPs, pain clinic regulations, medical marijuana laws, and legal/operational medical marijuana dispensaries. ${ }^{53}$ Again, the results are similar with these controls, implying that triplicate and non-triplicate states did not adopt systematically different opioid policies post-1996 which can explain the discrepancy in the growth rate of overdose deaths.

In addition, we test for differences in PDMP strength over time across triplicate and nontriplicate states using an index introduced in Pardo (2017). ${ }^{54}$ Appendix Figure A10, shows differences in PDMP strength for non-triplicate states relative to triplicate states, selecting on

\footnotetext{
52 Results are similar if we only use electronic PDMP states as the comparison group.

${ }^{53}$ We code dates for must access PDMPs and pain clinic regulations using the Prescription Drug Abuse Policy System (PDAPS). Data on marijuana laws and dispensaries are from the RAND Marijuana Policy database (see Powell et al. (2018) and Williams et al. (2019)).

${ }^{54}$ Pardo (2017) introduces an index of PDMP strength for 1999-2015, aggregating several different PDMP dimensions (e.g., mandatory use, timely reporting, etc.) together and validating the metric by showing that increases in PDMP strength are related to reductions in opioid-related overdose rates.
} 
states that had any type of PDMP as of 1996. There is little difference in how PDMP strength evolved between triplicate and non-triplicate states, yet we found much larger growth in fatal overdoses in non-triplicate states relative to these other PDMP states, as shown above.

Finally, we compare initial access to substance abuse treatment in triplicate and nontriplicate states. States with more access to substance abuse treatment may have prevented rising overdose death rates following OxyContin's introduction. Using the 1997 Uniform Facility Data Set (UFDS), ${ }^{55}$ we find that non-triplicate states had 3.938 treatment facilities per 100,000 people; triplicate states had 4.000. The difference is statistically insignificant ( $\mathrm{p}$-value $=0.94$ ).

\subsubsection{Deaths of Despair}

Explaining the growth in fatal drug overdoses is a central theme of the "deaths of despair" hypothesis discussed in Case and Deaton $(2015,2017)$, which studies trends in drug overdoses, suicides, and alcohol-related liver mortality. The hypothesis suggests that we would have observed an increase in mortality even in the absence of a rise in opioid supply due to worsening cultural and economic factors. In this section, we study other non-opioid deaths of despair - specifically, suicides (excluding overdoses) and alcohol-related liver deaths.

Appendix Figure A11 presents the event study estimates. We observe little evidence, especially of the same magnitude and significance as the overdose death effects, of differential rises in suicide rates or alcohol-related liver deaths by triplicate status. Suicides, excluding drug overdoses, trend upward in the non-triplicate states relative to the triplicate states beginning in the pre-period and continuing through the end of the sample period (Panel A). Alcohol-related liver deaths also exhibit pre-existing trends that continue throughout the period with some evidence of a flattening around 2001 (Panel B). ${ }^{56}$ Because both of these outcomes have noticeable systematic trends prior to 1996, we also show detrended estimates in Panels C and D of Appendix Figure A11. ${ }^{57}$ Overall, we find little evidence that other deaths of despair follow the same patterns as drug overdose deaths across triplicate and non-triplicate states, suggesting

\footnotetext{
${ }^{55}$ UFDS is a predecessor to the N-SSATS which records all known private and public substance abuse treatment facilities. The 1997 UFDS is the first year available from SAMHSA so we assume that treatment facilities did not open or close immediately due to differential OxyContin exposure.

${ }^{56}$ In principle, we might expect a delayed effect for alcohol-related liver deaths relative to those observed for overdoses if they were both driven by systematic differences in "despair" given the nature of these deaths. However, we do not observe an immediate or delayed increase in alcohol-related liver mortality.

${ }^{57} \mathrm{We}$ residualize the outcomes using estimated linear pre-trends (by triplicate status) using only the pre-1996 data (see Goodman-Bacon, 2016 for an equivalent use of this approach).
} 
that the differential supply and access to opioids played a crucial independent role in the opioid crisis. Moreover, the lack of a decline in suicides and alcohol-related liver mortality of the same magnitude suggests that fatal opioid overdoses were not substitutes for these types of deaths.

\subsubsection{Additional Robustness Tests}

We conducted numerous additional robustness tests that are presented and discussed in Appendices B-D. We briefly summarize our findings here. First, our results are unchanged if we account for changes in economic conditions (see Appendix B). We obtain similar estimates when we control for the unemployment rate or exogenous shocks to economic conditions as proxied by exposure to the decline in manufacturing (Charles et al., 2019), changes in labor demand (Betz and Jones, 2018), and exposure to trade liberalization policies (Pierce and Schott, forthcoming). Additionally, we find that the estimates are similar if we interact a set of our timevarying covariates with year indicators to permit the control variables to have different effects over time.

Second, the results are not affected by differential exposure to the crack epidemic, which ended shortly before the introduction of OxyContin. Our main results are similar when we exclude overdoses involving cocaine. The results are also unaffected if we exclude fatal overdoses involving unspecified narcotics.

Third, our estimates and statistical significance are robust to alternative inference methods (see Appendix C). We estimate confidence intervals using traditional clustered (by state) standard errors. This approach produces tighter confidence intervals than the restricted wild bootstrap method. We also conduct permutation tests. These tests show that it is statistically rare to observe our main mortality results when we randomly assign triplicate status to other combinations of states.

Finally, the estimated effects are similar if we use synthetic control estimation (see Appendix D), further suggesting that the effects are not driven by pre-existing differences in levels or trends.

\section{Mechanisms}

The above results show consistent evidence that non-triplicate states were more exposed to the introduction of OxyContin, causing a large and enduring increase in drug overdose deaths. 
In this section, we explore possible mechanisms for what drove the differential exposure to OxyContin. There are two possible channels. First, the triplicate programs themselves may have independently deterred OxyContin adoption. The effects of these programs could persist over the long run if the programs caused states to develop a prescribing culture that discouraged the use of strong opioids. Second, the reduced marketing by Purdue Pharma in triplicate states may have lessened OxyContin exposure. It is difficult to disentangle these two mechanisms because the recommendation to not promote OxyContin in triplicate states was based on the belief that triplicate programs would lead to low rates of OxyContin adoption.

We conduct two tests to provide evidence on these mechanisms. In the first test, we compare triplicate states to other states which also had low prescribing rates of oxycodone prior to 1996. In a second test, we compare the five triplicate states to the two former triplicate states that had discontinued their programs prior to 1996. In both tests, we find that the five triplicate states have uniquely low exposure to OxyContin and drug overdose rate growth even when compared to states with more comparable prescribing cultures. This evidence supports the role of Purdue Pharma's marketing rather than cultural factors and entrenched prescribing habits in explaining OxyContin exposure and mortality patterns.

Our first test is shown in Appendix Figure A12. We replicate the main event study analysis but limit the sample to the five triplicate states and the five non-triplicate states with the lowest oxycodone prescribing rates $^{58}$ in the 1991-1995 Medicaid data (see Appendix Table A2 for the list of states). For both OxyContin exposure (Panel A) and overdose death rates (Panel B), the estimates are remarkably similar to the main results of the paper despite the additional noise due to the smaller sample size. ${ }^{59}$ Triplicate states adopted OxyContin at much lower rates and had uniquely low overdose death growth compared to non-triplicate states that initially had similar prescribing habits.

Next, we compare the five triplicate states to only the two former triplicate states (Michigan and Indiana), which had triplicate programs prior to OxyContin's introduction but eliminated them in 1994. These former triplicate states serve as useful counterfactuals since they

\footnotetext{
58 This metric is highly-correlated with oxycodone prescriptions divided by oxycodone plus hydrocodone prescriptions, and the results are similar when using this metric. This alternative metric has the advantage of accounting for differences in opioid prescribing more generally, but we do not find that it matters empirically. ${ }^{59}$ We have also operationalized this test in alternative ways such as estimating the main event study for all states while controlling for a linear index of the 1991-1995 Medicaid prescribing rate interacted with time indicators, and the conclusions of this exercise are not meaningfully affected.
} 
would have developed similar prescribing cultures. Indeed, they were among the lowest prescribers of oxycodone prior to OxyContin's introduction (see Appendix Table A2) and this continued in 1995 after they had eliminated their triplicate programs (see Appendix Table A3). ${ }^{60}$

In Panel A of Figure 8, we present the cross-sectional differences in OxyContin distribution using ARCOS data for the two former triplicate states compared to the five triplicate states (dashed line). The solid line shows the differences for never-triplicate states compared to the five triplicates. The former triplicates adopted OxyContin at a substantially higher rate than the triplicate states, although at a lower rate than the never-triplicates (in 2000, the difference is 0.50 morphine equivalent doses per capita in former triplicates versus 0.73 in never triplicates). ${ }^{61}$

Overall, the former triplicates appear more like the never-triplicates than the triplicate states in terms of OxyContin exposure. In Panel B, we estimate the main event study for drug overdose deaths showing separate coefficients for the former-triplicates and never-triplicates. Both groups of non-triplicate states experience similar mortality trajectories when compared to triplicate states. Thus, states with triplicate programs at the time of OxyContin's launch experienced uniquely low exposure to OxyContin and drug overdose death rates even when compared to states which had triplicate programs just two years prior.

We draw two conclusions from these tests. First, prescribing culture alone cannot explain the lack of OxyContin adoption and fatal overdose patterns in triplicate states. States with similarly low initial levels of oxycodone prescribing as triplicate states still had much higher rates of OxyContin adoption and overdose deaths. Second, states that had recently discontinued triplicate programs adopted OxyContin and experienced subsequent overdose death growth of similar magnitude as other non-triplicate states. ${ }^{62}$ Our five triplicate states, however,

\footnotetext{
${ }^{60}$ Table A3 shows Medicaid oxycodone prescribing rates for 1995, after Indiana and Michigan ended their triplicate programs. We still see both states near the bottom of the distribution, suggesting persistence in prescribing culture as they did not respond to the repeal of the triplicate programs by dramatically increasing oxycodone prescribing. ${ }^{61}$ OxyContin distribution is skewed so the mean is much higher than the median. We plot median per capita OxyContin supply for 2000-2016 in Appendix Figure A13. The former-triplicates and never-triplicates have very similar median OxyContin supply (in 2000, the difference is just 0.1 per capita MEDs), but both have much higher supply than triplicates.

${ }^{62}$ One alternative explanation for this pattern is that the former triplicate states had active triplicate programs for shorter periods of time than the triplicate states (i.e., CA adopted in 1939). This reduced exposure to the program may have lessened the persistence of any developed prescribing culture. However, as noted previously, Indiana and Michigan had oxycodone prescribing rates that were similar to the five triplicate states even in 1995 after elimination of their program (see Appendix Table A3), suggesting that the triplicate programs induced low and persistent oxycodone prescribing habits even in that shorter time period. Moreover, Texas, which adopted its triplicate program in the same decade as Indiana and Michigan, experienced much lower overdose death growth than these states.
} 
continued to experience relatively low rates of OxyContin use and overdose death rates even after eliminating their own triplicate programs in the late 1990s and early 2000s, suggesting that the 1996 triplicate states were differentially treated compared to the former triplicate states. An explanation for these differences is differential marketing exposure targeted to non-triplicate states at the time of OxyContin's launch.

Our results suggest that the lack of exposure to OxyContin in the years after its introduction had enduring effects that can explain differences in drug overdose death rates even today. The enduring nature of these effects is hard to reconcile with the prescribing culture mechanism hypothesis since states with a history of low oxycodone prescribing and former triplicates adopted OxyContin at high rates and experienced lasting increases in overdoses. Purdue Pharma's marketing strategy, however, could explain some of the long-term effects of differential initial exposure. Internal Purdue Pharma documents show that their strategy was to call and visit the top OxyContin prescribers. The early budget plans for Purdue Pharma annually dictated that the sales force target calls to the top 1 to 3 (depending on the year) deciles of physicians in terms of past prescribing behavior. Documents from the Massachusetts case against Purdue Pharma includes additional evidence from internal communications discussing this targeting behavior and how it extended until 2018. ${ }^{63}$ This marketing strategy would generate serial correlation in OxyContin prescribing given differential initial exposure. Since triplicate states would have initially attracted less marketing because of their lower prescribing, they would continue to receive less marketing in future time periods and low prescribing would persist.

While we have been unable to obtain data on state-level physician detailing behavior from the 1990s to study these differences directly, we can study Purdue Pharma detailing in 2013-2016 using the CMS Open Payments Data as a measure of persistent differences in detailing. This database reports payments to physicians for meals, travel, and gifts regarding promotion of specific drugs. We calculate the total payments to physicians per capita over these years for OxyContin. Figure 9 shows that there are large (and statistically significant) differences between triplicate and non-triplicate states. Never-triplicate states receive $42-72 \%$

\footnotetext{
${ }^{63}$ For example, "McKinsey recommended doubling down on Purdue Pharma's strategy of targeting high prescribers for even more sales calls..." (p. 212 of Commonwealth of Massachusetts, 2018). Purdue announced in 2018 that they stopped all opioid promotional activities targeting physicians.
} 
more payments per capita than triplicates states in each year. As an alternative metric, we scale the OxyContin-specific payments by total payments to account for state-level differences in promotional activities. We present these measures in Appendix Figure A14. The gap between triplicates and non-triplicates grows even further when using this metric. Similar to other outcomes, the former-triplicates are much more similar to the never-triplicate states than the five triplicate states. The evidence of promotional activities for opioids responding to state-level PDMPs is also consistent with findings in Nguyen et al. (2019) concerning more recent adoption of mandatory access PDMPs in the 2010s.

This evidence is consistent with persistence based on serial correlation in marketing practices: greater initial marketing by Purdue Pharma in non-triplicate states, leading to higher rates of prescribing which, in turn, led Purdue Pharma to target those places more in later years. It is otherwise difficult to explain why the triplicate states as of 1996 experienced such enduringly low rates of overdose death growth, but states that had eliminated their programs just two years prior experienced overdose death trends almost identical to never-triplicate states. If triplicate programs themselves had such enduring effects, then we would expect to observe similar (or at least muted) effects for the former triplicate states as well.

One open question is why Purdue Pharma would dedicate resources to eliminating triplicate programs and then not make a major marketing push in those states when those programs were eliminated. Unfortunately, there are limits to our ability to discern Purdue Pharma's promotional strategies. However, we do not observe a large jump in OxyContin or oxycodone prescriptions when states eliminate their triplicate programs. ${ }^{64}$ In Appendix Figure A15, we plot the estimates from an event study examining Medicaid prescriptions around the triplicate repeal dates (conditional on state and time fixed effects). We find a downward trend over time, consistent with the general separation between non-triplicate and triplicate states over time and no independent effect of triplicate repeal. While this does not rule out subsequent targeting of marketing to the triplicate states, it suggests that there may not have been a dramatic increase in marketing intensity or that the content (and efficacy) of marketing had changed by this point in time due to growing knowledge of OxyContin abuse and the government's scrutiny of the company's misleading advertising practices. The possibility that advertising did not

\footnotetext{
${ }^{64}$ We use Medicaid prescriptions so we can include all triplicate states in the analysis. With the ARCOS OxyContin data, only 2 states have data for years prior to repeal (though the patterns are similar using ARCOS).
} 
increase to non-triplicate levels is also supported by the findings from the Open Payments data, discussed previously, showing that advertising differences across triplicate and non-triplicate have continued until the present day.

Overall, the results in this section are consistent with Purdue Pharma's initial marketing decisions contributing to persistent differences in overdose deaths across states. The evidence of triplicate programs independently inducing differential and enduring prescribing of OxyContin is not well supported.

\section{$7 \quad$ Discussion and Conclusion}

Despite the importance of the opioid crisis and the desire to understand its origins, there is little empirical work exploring its initial causes. We study the effects of the introduction of OxyContin in 1996, exploiting early variation in its promotion and market entry based on preexisting state policies known as triplicate prescription programs. These state policies were adopted decades earlier and became outdated soon after OxyContin's launch. However, their initial deterrence of OxyContin promotion and use had long-term effects on overdoses in these states, dramatically decreasing overdose death rates even today.

Our results imply striking differences throughout the opioid crisis stemming from variation in initial policy conditions. States with more exposure to OxyContin's introduction experienced higher growth in overdose deaths in almost every year since 1996. In the first wave of the opioid crisis, this disproportionate growth is driven primarily by deaths from prescription opioids. After the reformulation of OxyContin in 2010, overdose deaths involving heroin and fentanyl also play a critical role. Our estimates (using Table 3, Column 3) show that nontriplicate states would have experienced 4.49 fewer drug overdose deaths per 100,000 on average from 1996-2017 if they had been triplicate states and 3.04 fewer opioid overdose deaths per $100,000{ }^{65}$ We first consider the implications of these changes on overdose rate levels for this time period. Over this time period, non-triplicates had an average of 12.32 fatal overdoses per 100,000 annually and 6.97 of those involved opioids. This implies that if non-triplicate states had the same initial level of exposure to OxyContin as triplicate states, they would have had $36 \%$

\footnotetext{
${ }^{65}$ We take the three post estimates and weight each by the sum of the non-triplicate population size over the relevant time periods (i.e., longer time periods receive more weight).
} 
fewer drug overdose deaths and $44 \%$ fewer opioid overdose deaths on average in each year from 1996-2017.

We use our results to provide a back-of-the-envelope calculation of how much of the dramatic growth in drug overdose deaths can be accounted for by the introduction and marketing of OxyContin. On average, the national drug overdose death rate increased by 6.89 deaths per 100,000 since 1996, comparing the mean during the 1996-2017 time period (11.33 deaths per $100,000)$ relative to the 1991-1995 baseline mean (4.44). The additional exposure to OxyContin's launch and marketing for non-triplicate states led to 4.49 more deaths per 100,000, which is equivalent to $65 \%(4.49 / 6.89)$ of the national growth in overdose death rates since 1996. As an additional benchmarking exercise, we consider the additional deaths that could be attributed to an increase in initial OxyContin exposure moving from no exposure to the national level of exposure. To make this calculation, we need to scale our mortality results by the difference in OxyContin exposure between non-triplicate and triplicate states. ${ }^{66}$ This extrapolation suggests that moving from no OxyContin exposure to the national average would lead to 5.56 more deaths per 100,000 , which is $81 \%$ of the rise in the overdose death rate since 1996. We note that this extrapolation is far out-of-sample since no part of the United States was unexposed, so we interpret it with caution. However, these calculations suggest that exposure to OxyContin may explain a large share of the growth in drug overdose deaths since the mid-1990s.

The estimates are large because they capture both the direct and indirect consequences of initial exposure to OxyContin, including spillovers of OxyContin promotion to other opioid drugs and transitions to heroin and fentanyl in the later waves of the epidemic. They also internalize downstream indirect effects of OxyContin's introduction on the behaviors of other entities in the supply chain — distributors, pharmacies, and doctors - which may have further

\footnotetext{
${ }^{66}$ For the purposes of this extrapolation, we assume that differences in initial OxyContin supply serve as a summary metric reflecting differences in exposure from the launch and marketing. Our extrapolation exercise can be summarized as follows. First, we scale our mortality estimate by the additional initial OxyContin exposure experienced in non-triplicate states. We calculate the difference in OxyContin exposure between non-triplicate and triplicate states as 1.09 morphine equivalent doses (MEDs) per capita, using the first available year of ARCOS data in 2000. This implies that one additional MED per capita of initial OxyContin exposure led to an additional 4.12 (4.49/1.09) drug overdose deaths per 100,000 annually. Next, we linearly extrapolate from this estimate to calculate the effects of moving from the national average initial OxyContin exposure (1.35 MEDs per capita in 2000) to no exposure. This implies that there would be $5.56(4.12 * 1.35)$ fewer drug overdose deaths per 100,000 each year if there had been no OxyContin exposure nationally. Relative to the average national growth in the drug overdose death rate of 6.89 deaths per 100,000 from 1996-2017, this represents an 81\% (5.56/6.89) decline. Alternative scaling factors are also possible (e.g., ARCOS for the full 2000-2016 period, MEPS prescriptions, Medicaid prescriptions) and typically generate larger estimates.
} 
amplified OxyContin's effects. Prior evidence showed that the removal of the abusable formulation of OxyContin led to a striking national rise in heroin deaths after 2010. Consistent with that evidence, this paper suggests that the introduction of OxyContin had significant effects on overdose deaths from the beginning of the opioid crisis.

Our findings do not rule out the possibility that economic and cultural factors also contributed to a meaningful share of the rise in drug-related mortality. However, we find that the effects of the supply-side shock studied in this paper persist even when we account for demand-side factors. While these results help quantify the harms associated with OxyContin exposure, our analysis does not speak to the potential benefits of improved opioid access through the introduction of OxyContin. Opioids may be effective pain management tools in some cases, and we do not attempt to estimate the direct or indirect gains from pain reduction stemming from OxyContin's launch.

Finally, the evidence in this paper is consistent with Purdue Pharma's marketing practices playing an important role in explaining growth in drug overdose rates. When triplicate states are compared to other states with similar prior oxycodone prescribing rates or even states which had just recently eliminated their triplicate programs, they still have uniquely low overdose death rate growth. This suggests that it is less likely that triplicate programs independently influenced OxyContin adoption. Instead, the evidence is more consistent with differences in local marketing leading to persistent differences in overdose death growth due to the nature of Purdue Pharma's marketing techniques. Overall, we find strong evidence that the marketing practices of OxyContin interacted with state-level policy conditions led to dramatically reduced overdose death rates in triplicate states. By deterring OxyContin's widespread introduction in 1996, triplicate programs appear to have protected some states against the long-term fatal overdose trends experienced by most other states. 


\section{References}

Alexander, Monica J, Mathew V Kiang, and Magali Barbieri, "Trends in black and white opioid mortality in the United States, 1979-2015," Epidemiology (Cambridge, Mass.), 2018, 29 (5), 707.

Alpert, Abby, David Powell, and Rosalie Liccardo Pacula, "Supply-side drug policy in the presence of substitutes: Evidence from the introduction of abuse-deterrent opioids," American Economic Journal: Economic Policy, 2018, 10 (4), 1-35.

Barnett, Michael L, Andrew R Olenski, and Anupam B Jena, "Opioid-prescribing patterns of emergency physicians and risk of long-term use," New England Journal of Medicine. 2017, 376 (7): 663-673.

Berina, Leslie F, Brock G Guernsey, James A Hokanson, William H Doutre, and Lonnie E Fuller, "Physician perception of a triplicate prescription law." American Journal of HealthSystem Pharmacy, 1985, 42 (4), 857-860.

Betz, Michael R and Lauren E Jones, "Wage and employment growth in America's drug epidemic: Is all growth created equal?" American Journal of Agricultural Economics, 2018, 100 (5), 1357-1374.

Brewer, Mike, Thomas F Crossley, and Robert Joyce, "Inference with difference-indifferences revisited," Journal of Econometric Methods, 2018, 7 (1).

Buchmueller, Thomas C, and Colleen Carey, "The effect of prescription drug monitoring programs on opioid utilization in Medicare." American Economic Journal: Economic

Policy, 2018, 10 (1), 77-112.

Cameron, A Colin, Jonah B Gelbach, and Douglas L Miller, "Bootstrap-based improvements for inference with clustered errors," The Review of Economics and Statistics, 2008, 90 (3), 414427.

Case, Anne and Angus Deaton, "Rising morbidity and mortality in midlife among white nonHispanic Americans in the 21st century," Proceedings of the National Academy of Sciences, 2015, 112 (49), 15078-15083.

_ and _, "Mortality and morbidity in the 21 st century," Brookings Papers on Economic Activity, 2017, 2017, 397-443.

Centers for Disease Control and Prevention (CDC). "Overdose deaths involving prescription opioids among Medicaid enrollees-Washington, 2004-2007.” MMWR: Morbidity and Mortality Weekly Report, 2009, 58 (42), 1171-1175.

Charles, Kerwin Kofi, Erik Hurst, and Mariel Schwartz, "The transformation of manufacturing and the decline in US employment." NBER Macroeconomics Annual 33, no. 1, 2019: 307-372.

Ciccarone, Daniel, "Fentanyl in the US heroin supply: a rapidly changing risk environment." The International Journal on Drug Policy. 2017. 46, 107-111. 
Cicero, Theodore J, James A. Inciardi, and Alvaro Munoz, "Trends in Abuse of OxyContin ${ }^{\circledR}$ and Other Opioid Analgesics in the United States: 2002-2004," The Journal of Pain. 2005, 6 (10), 662-672.

Commonwealth of Massachusetts, "Commonwealth of Massachusetts v. Purdue Pharma L.P., Purdue Pharma Inc., Richard Sackler, Theresa Sackler, Kathe Sackler, Jonathan Sackler, Mortimer D.A. Sackler, Beverly Sackler, David Sackler, Ilene Sackler Lefcourt, Peter Boer, Paulo Costa, Cecil Pickett, Ralph Snyderman, Judy Lewent, Craig Landau, John Stewart, and Mark Timney." June 12, 2018. https://www.mass.gov/files/documents/2018/06/12/Purdue\%20Complaint\%20FILED.pdf

Conley, Timothy $\mathbf{G}$ and Christopher R Taber, 'Inference with 'difference in differences' with a small number of policy changes," The Review of Economics and Statistics, 2011, 93 (1), 113125.

DeBruyne, Nese F. "American War and Military Operations Casualties: Lists and Statistics." Congressional Research Service. 2018.

Dyer, Owen, “US Life Expectancy Falls for Third Year in a Row,” BMJ, 2018, 363, k5118.

Evans, William N, Ethan Lieber, and Patrick Power, "How the Reformulation of OxyContin Ignited the Heroin Epidemic," Review of Economics and Statistics. 2019. 101(1), 1-15.

Fernandes, Jessie C, David Campana, Todd S Harwell, and Steven D Helgerson. "High mortality rate of unintentional poisoning due to prescription opioids in adults enrolled in Medicaid compared to those not enrolled in Medicaid in Montana." Drug and Alcohol Dependence, 2015, 153, 346-349.

Fishman, Scott M, Jennifer S Papazian, Susana Gonzalez, Paul S Riches, and Aaron Gilson, "Regulating opioid prescribing through prescription monitoring programs: Balancing drug diversion and treatment of pain," Pain Medicine, 2004, 5 (3), 309-324.

Government Accountability Office (GAO), "Prescription Drugs: OxyContin Abuse and Diversion and Efforts to Address the Problem: Report to Congressional Requesters," DIANE Publishing, 2003.

Goodman-Bacon, Andrew, "The long-run effects of childhood insurance coverage: Medicaid implementation, adult health, and labor market outcomes." No. w22899. National Bureau of Economic Research, 2016.

Green, Carla A, Nancy A Perrin, Shannon L. Janoff, Cynthia I Campbell, Howard D Chilcoat, and Paul M Coplan, "Assessing the accuracy of opioid overdose and poisoning codes in diagnostic information from electronic health records, claims data and death records." Pharmacoepidemiology \& Drug Safety. 2017, 26 (5), 509-517.

Groups Plus, "Purdue Pharma Company Focus Group Research \& Findings Oxycontin for NonCancer Pain Management," Technical Report 1995.

Hartzema, Abraham G, Miquel S Porta, Hugh H Tilson, Sergio G Zullich, Thaddeus H Grasela Jr, Jill B Fiedler-Kelly, and Francis M Gengo, "Impact of triplicate prescription 
program on psychotropic prescribing patterns in long-term care facilities," Annals of Pharmacotherapy, 1992, 26 (4), 539-546.

Hollingsworth, Alex, Christopher J Ruhm, and Kosali Simon, "Macroeconomic conditions and opioid abuse," Journal of Health Economics, 2017, 56, 222-233.

Horwitz, Jill, Corey S. Davis, Lynn S. McClelland, Rebecca S. Fordon, and Ellen Meara, "The problem of data quality in analyses of opioid regulation: The case of prescription drug monitoring programs." National Bureau of Economic Research, No. w24947, 2018.

IMS Institute for Healthcare Informatics, "The use of medicines in the United States: Review of 2010," 2011.

Jones, Mark R, Omar Viswanath, Jacquelin Peck, Alan D Kaye, Jatinder S Gill, and Thomas T Simopoulos. "A brief history of the opioid epidemic and strategies for pain medicine," Pain and Therapy. 2018, 7 (1), 13-21.

Joranson, David E, Grant M Carrow, Karen M Ryan, Linda Schaefer, Aaron M Gilson, Patricia Good, John Eadie, Susan Peine, and June L Dahl, "Pain management and prescription monitoring," Journal of Pain and Symptom Management, 2002, 23 (3), 231-238.

Khan, Nazleen F, Brian T Bateman, and Joan E Landon. "Association of opioid overdose with opioid prescriptions to family members," JAMA Internal Medicine. 2019, 179 (9), 11861192 .

Kolodny, Andrew, David T Courtwright, Catherine S Hwang, Peter Kreiner, John L Eadie, Thomas W Clark, and G Caleb Alexander, "The prescription opioid and heroin crisis: A public health approach to an epidemic of addiction," Annual Review of Public Health, 2015, 36, 559-574.

Melzack Ronald. "The tragedy of needless pain.” Scientific American.1990, 262 (2), 27-33.

Morgan John P. "American opiophobia: customary underutilization of opioid analgesics." Adv AlcoholSubst Abuse. 1985, 5 (1-2), 163-73.

Nguyen, Thuy D, W David Bradford, and Kosali I Simon, "How do opioid prescribing restrictions affect pharmaceutical promotion? Lessons from the Mandatory Access Prescription Drug Monitoring Programs.” NBER Working Paper 2019.

Pardo, Bryce, "Do more robust prescription drug monitoring programs reduce prescription opioid overdose?" Addiction, 2017, 112(10): 1773-1783

Pardo, Bryce, Jonathan P. Caulkins, Beau Kilmer, Rosalie Liccardo Pacula, Peter Reuter, and Bradley D. Stein. "The synthetic opioid surge in the United States." RAND Publication 2019. https://www.rand.org/pubs/research_reports/RR3116.html

Phillips, Donald M, "JCAHO pain management standards are unveiled," JAMA, 2000, 284 (4), 428-429.

Pierce, Justin and Peter Schott. "Trade liberalization and mortality: Evidence from U.S. counties." American Economic Review: Insights, forthcoming. 
Porter J, Jick H. "Addiction rare in patients treated with narcotics." New England Journal of Medicine. 1980, 302 (2), 123.

Powell, David, Rosalie Liccardo Pacula, and Mireille Jacobson, "Do medical marijuana laws reduce addictions and deaths related to pain killers?" Journal of Health Economics, 2018, 58, 2942.

Purdue Pharma, "OxyContin Launch Plan," Technical Report 1995.

_ , "1997 Budget Plan," Technical Report 1997.

_ , "1998 Budget Plan," Technical Report 1998.

_ , "1999 Budget Plan," Technical Report 1999.

_, "2001 Budget Plan," Technical Report 2001.

_ , "2002 Budget Plan," Technical Report 2002.

Quinones, Sam. "Dreamland: The True Tale of America's Opiate Epidemic." New York: Bloomsbury Publishing. 2015.

Roodman, David Malin, James G MacKinnon, Morten Ørregaard Nielsen, and Matthew Webb, "Fast and wild: bootstrap inference in Stata using Boottest," Technical Report, Queen's Economics Department Working Paper 2018.

Ruggles, Steven, Sarah Flood, Ronald Goeken, Josiah Grover, Erin Meyer, Jose Pacas, and Matthew Sobek, "IPUMS USA: Version 8.0 [dataset]," 2018.

Ruhm, Christopher J, "Corrected US opioid-involved drug poisoning deaths and mortality rates, 1999-2015." Addiction, 2018, 113 (7), 1339-1344.

Ruhm, Christopher J, "Drivers of the fatal drug epidemic." Journal of Health Economics 64, 2019, 25-42.

Scholl, Lawrence, Puja Seth, Mbabazi Kariisa, Nana Wilson, and Grant Baldwin, "Drug and opioid-involved overdose deaths-United States, 2013-2017." Morbidity and Mortality Weekly Report 67, no. 5152, 2019: 1419.

Sharp, Mark J., and Thomas A. Melnik. "Poisoning deaths involving opioid analgesics-New York State, 2003-2012." MMWR. Morbidity and Mortality Weekly Report, 2015, 64 (14): 377.

Sigler, KA, BG Guernsey, NB Ingrim, AS Buesing, JA Hokanson, E Galvan, and WH Doutre, "Effect of a triplicate prescription law on prescribing of Schedule II drugs," American Journal of Health-System Pharmacy, 1984, 41 (1), 108-111.

Simoni-Wastila, Linda and Wendy Toler, "State-Issued Prescription Forms," n.d. https://cdn.ymaws.com/www.safestates.org/resource/resmgr/imported/Simoni\%20Wastila.pdf, last accessed March 11, 2019.

Simoni-Wastila, Linda and Christopher Tompkins, "Balancing diversion control and medical necessity: The case of prescription drugs with abuse potential," Substance Use \& Misuse, 2001, $36(9-10), 1275-1296$. 
_, Dennis Ross-Degnan, Connie Mah, Xiaoming Gao, Jeffrey Brown, Leon E Cosler, Thomas Fanning, Peter Gallagher, Carl Salzman, and Stephen B Soumerai, “A retrospective data analysis of the impact of the New York triplicate prescription program on benzodiazepine use in Medicaid patients with chronic psychiatric and neurologic disorders," Clinical Therapeutics, 2004, 26 (2), 322-336.

Strategic Business Research, Inc., "OxyContin: Quantitative Research," Technical Report 1996.

Substance Abuse and Mental Health Services (SAMHSA) Center for Behavioral Health Statistics and Quality, "Treatment Episode Data Set (TEDS): 2001-2011. State Admissions to Substance Abuse Treatment Services," 2013.

University of Kentucky Center for Poverty Research, "UKCPR National Welfare Data, 19802017," 2018.

Van Zee, Art, "The promotion and marketing of OxyContin: Commercial triumph, public health tragedy," American Journal of Public Health, 2009, 99 (2), 221-227.

Venkataramani, Atheendar S and Paula Chatterjee, "Early Medicaid Expansions and Drug Overdose Mortality in the USA: A Quasi-Experimental Analysis." Journal of General Internal Medicine 34.1, 2019: 23-25.

Warner, Margaret, Li Hui Chen, Diane M Makuc, Robert N Anderson, and Arialdi M Miniño, "Drug poisoning deaths in the United States, 1980-2008," NCHS data brief, 2011, (81), $1-8$.

Webb, Matthew D, "Reworking wild bootstrap based inference for clustered errors," Technical Report, Queen's Economics Department Working Paper 2014.

Weintraub, Michael, Satesh Singh, Louise Byrne, Kumar Maharaj, and Laurence

Guttmacher, "Consequences of the 1989 New York State triplicate benzodiazepine prescription regulations," JAMA, 1991, 266 (17), 2392-2397.

Whitmire, J. Timothy, and Glenda Waslaski Adams, "Unintentional overdose deaths in the North Carolina Medicaid population: prevalence, prescription drug use, and medical care services." Center for Health Statistics, 2010.

https://schs.dph.ncdhhs.gov/schs/pdf/schs 162 web_081310.pdf, last accessed March 11, 2019

Williams, Jenny, Rosalie Liccardo Pacula, and Rosanna Smart, "De Facto or De Jure?

Ethnic Differences in Quit Responses to Legal Protections of Medical Marijuana Dispensaries." No. w25555. National Bureau of Economic Research, 2019.

World Health Organization (WHO), "Cancer Pain Relief," 1986. 


\section{Figures}

\section{Figure 1: National Drug Overdose Death Rates}

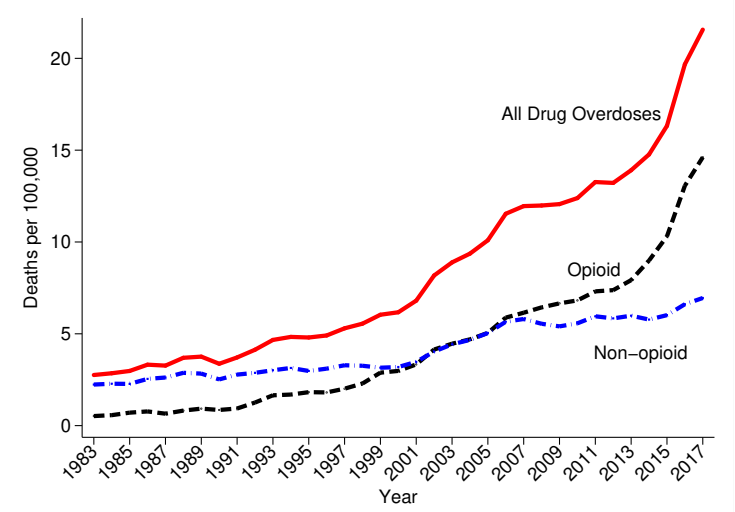

Notes: We use geocoded NVSS data to construct total overdose and opioid overdose deaths per 100,000. See Section 3.1 for ICD codes used in each period. Opioid overdoses are defined as overdoses which report opioid involvement (including natural/semisynthetic opioids, heroin, and synthetic opioids). Non-opioid overdoses are defined as overdoses that do not report opioid involvement.

Figure 2: Drug Overdose Death Rates By Triplicate State Status

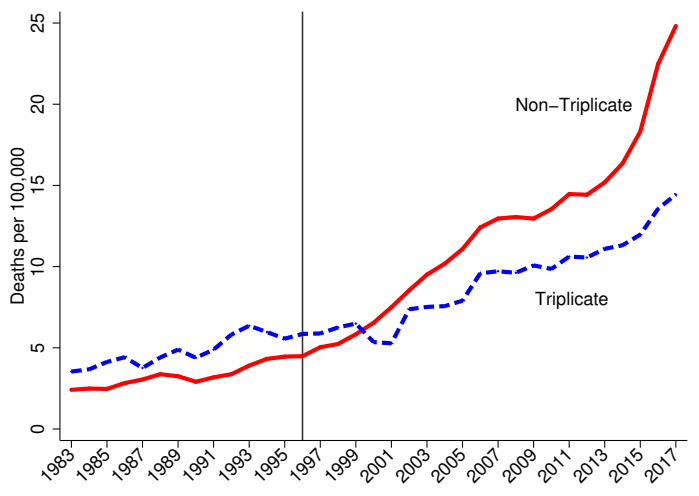

A: All Drug Overdose Deaths

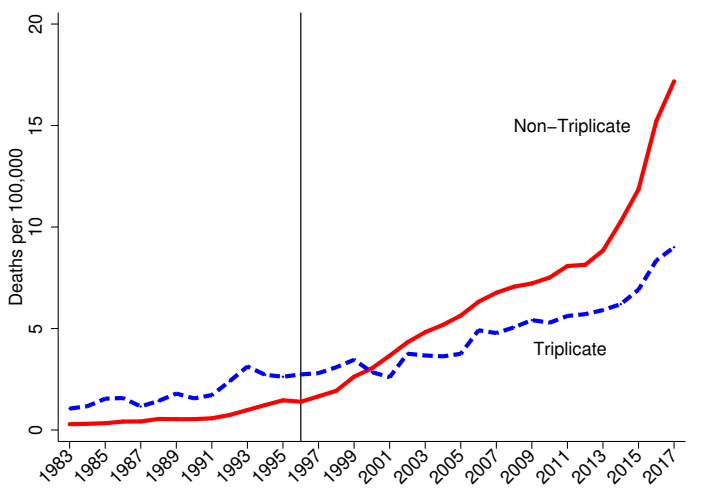

B: Opioid Overdose Deaths

Notes: We use geocoded NVSS data to construct total overdose and opioid overdose deaths per 100,000. See Section 3.1 for ICD codes used in each period. The vertical line marks the introduction of OxyContin in 1996. 
Figure 3: OxyContin Distribution and Prescriptions by Triplicate State Status

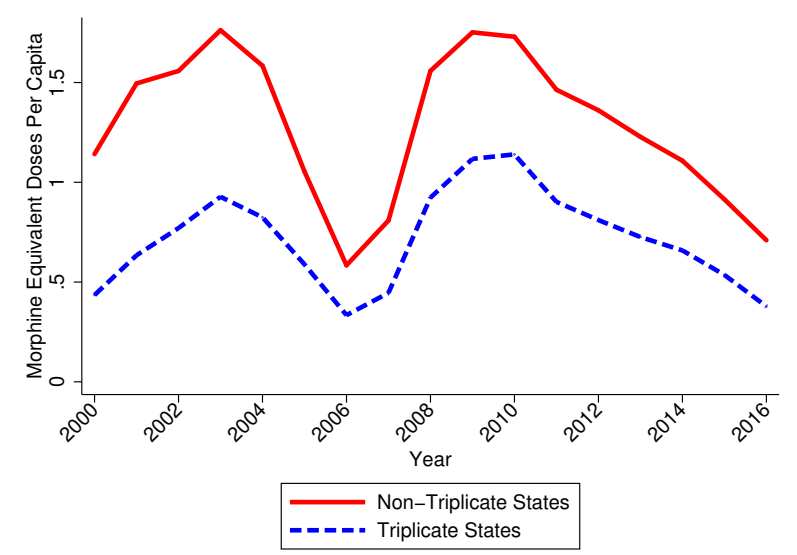

A: OxyContin Distribution (ARCOS)

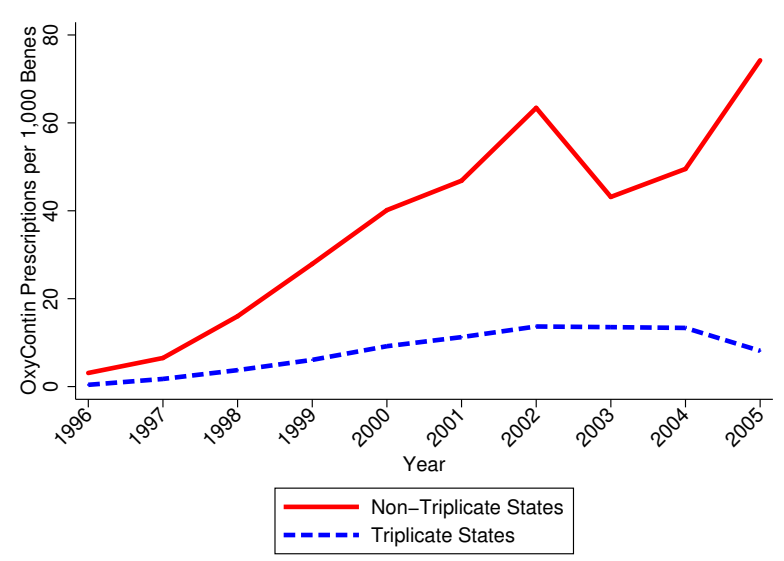

B: OxyContin Prescriptions (Medicaid)

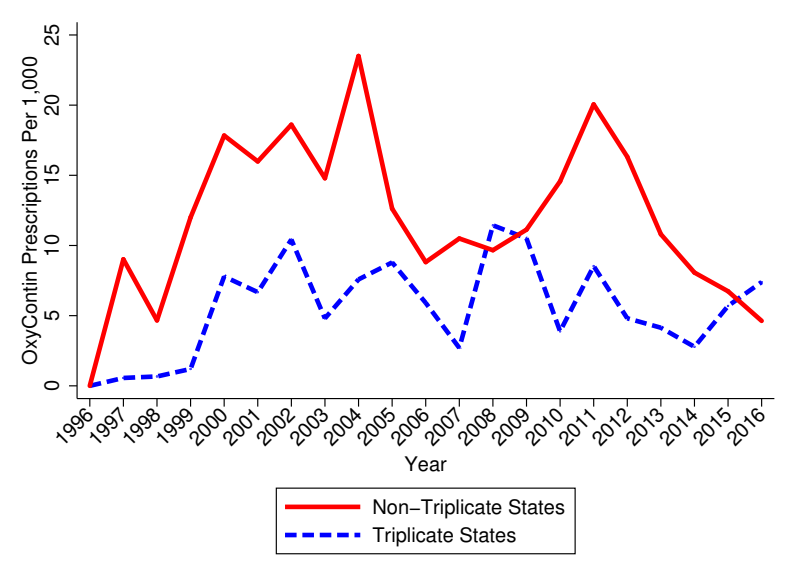

C: OxyContin Prescriptions (MEPS)

Notes: In Panel A, we use ARCOS data and construct morphine equivalent doses per capita. OxyContin data are only available for 2000-2016. In Panel B, we report the number of prescriptions per 1,000 beneficiaries from the Medicaid SDUD. We end this time series in 2005 due to the introduction of Medicare Part D. In Panel C, we report the number of prescriptions per 1,000 people in the MEPS. We use the MEPS survey weights. 
Figure 4: Oxycodone and Hydrocodone Distribution by Triplicate State Status

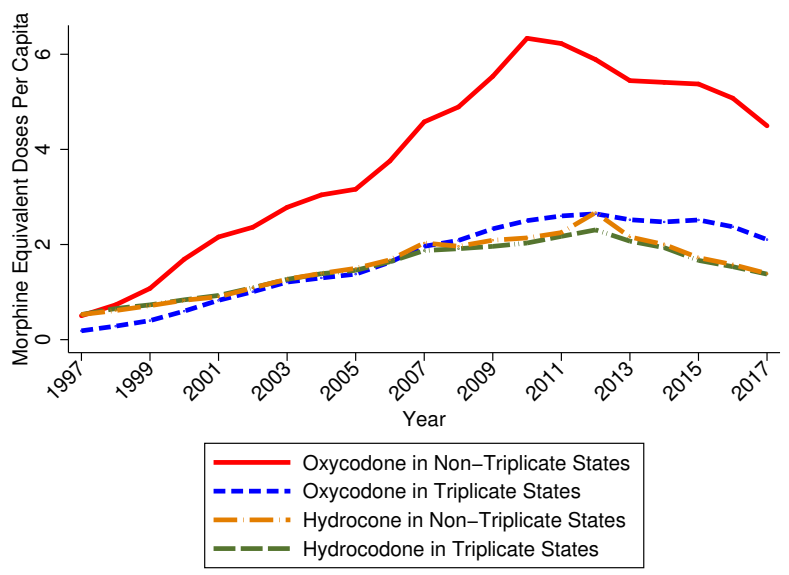

Notes: We use ARCOS data to construct morphine equivalent doses per capita by substance.

Figure 5: Non-Medical Use Rates by Triplicate State Status

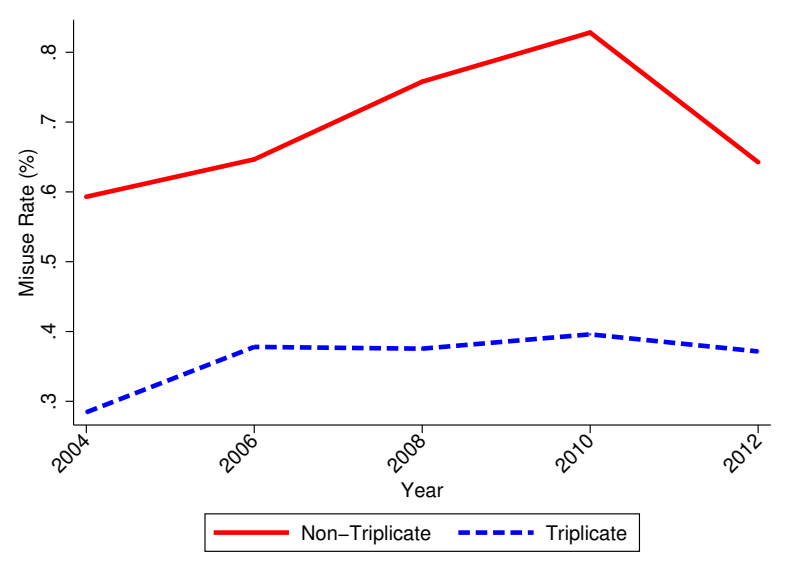

A: OxyContin

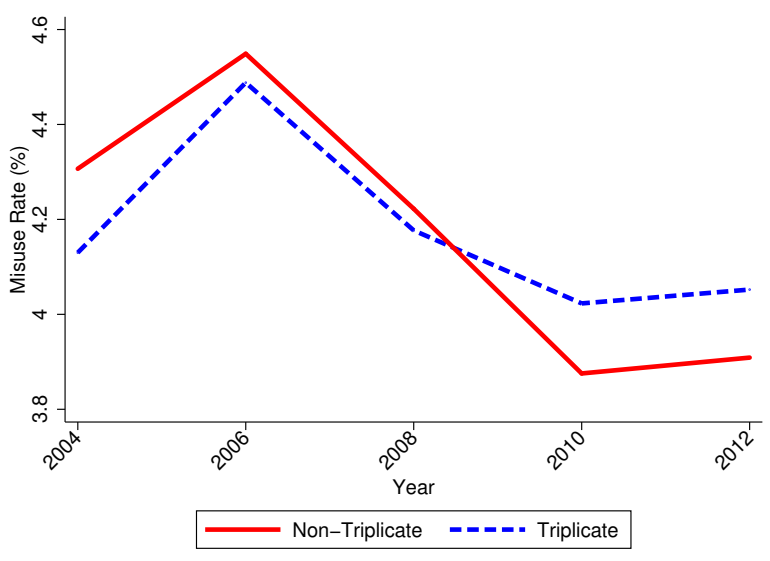

B: Pain Relievers excluding OxyContin

Notes: Misuse rates are calculated from the National Survey on Drug Use and Health using the years available. Each year refers to a two-year wave such that "2004" refers to 2004-2005, "2006" refers to 2006-2007, etc. 
Figure 6: Event Study: Drug Overdose Death Rate

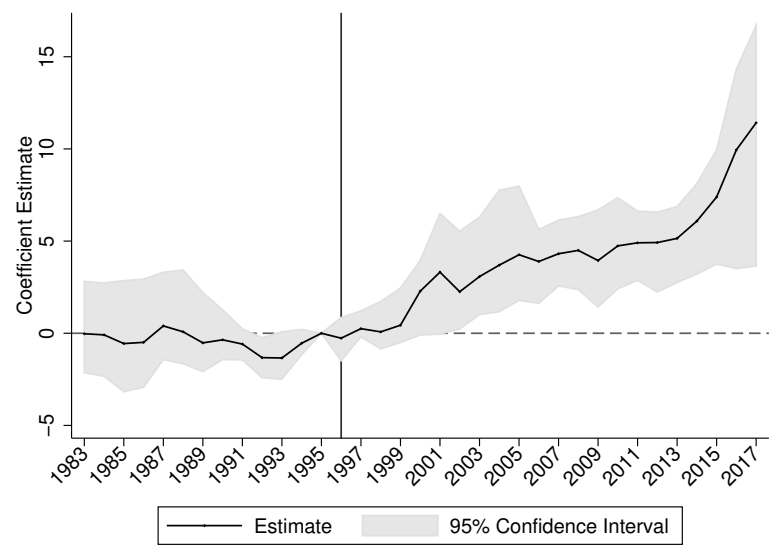

A: All Drug Overdose Deaths

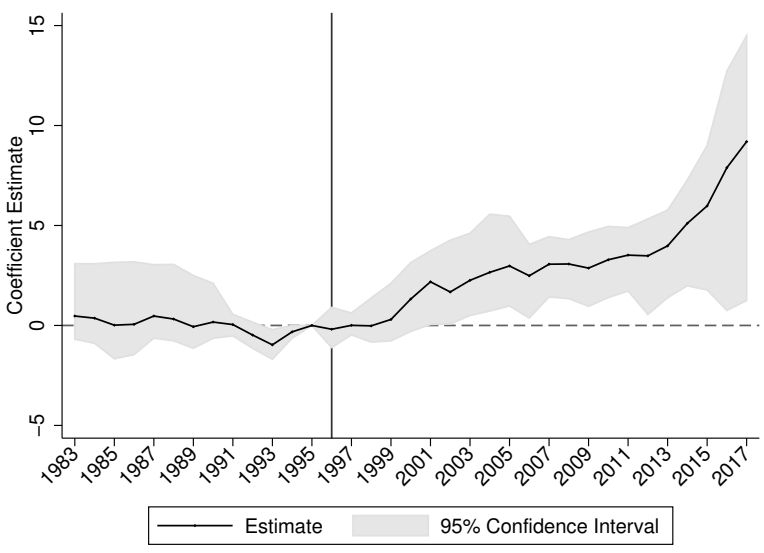

B: Opioid Overdose Deaths

Notes: We use geocoded NVSS data to construct overdoses and opioid overdose deaths per 100,000. See Section 3.1 for exact ICD codes used in each period. 95\% confidence intervals are generated using a clustered (at state) wild bootstrap. Estimates are normalized to 0 in 1995 . Weighted by population.

Figure 7: Drug Overdose Death Rate Changes: Triplicate States vs. Bordering States 1996-2005 Relative to 1986-1995
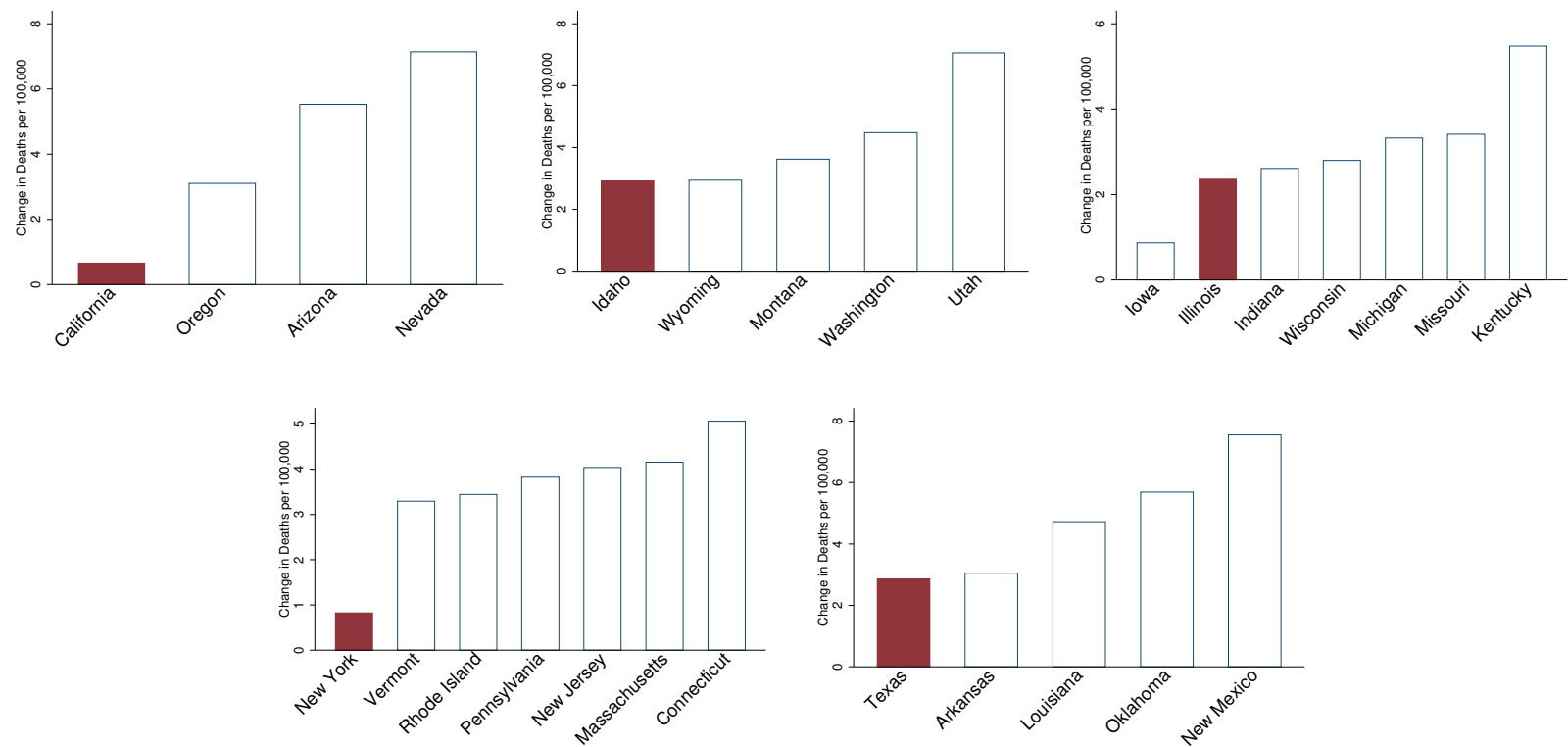

Notes: We construct the change in overdose deaths per 100,000 for 1996-2005 relative to 1986-1995. We plot this change for each triplicate state relative to its bordering states. 
Figure 8: Event Study: Comparing Former Triplicate States to Never Triplicate States

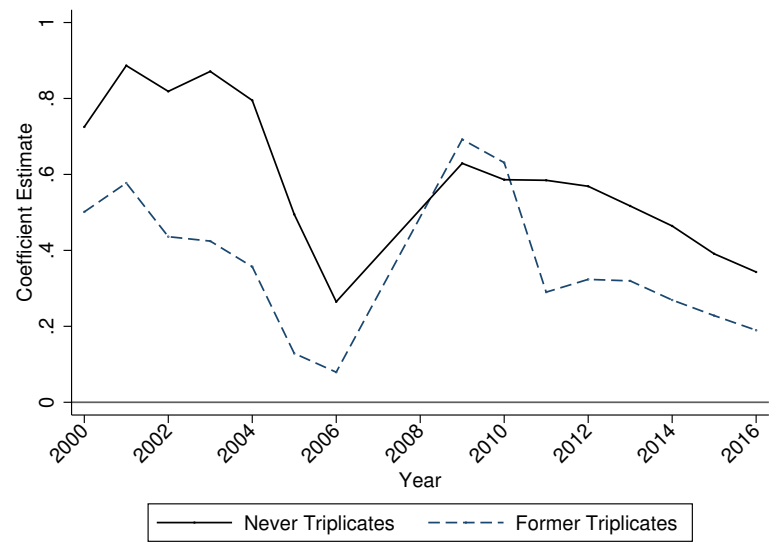

A: OxyContin Distribution

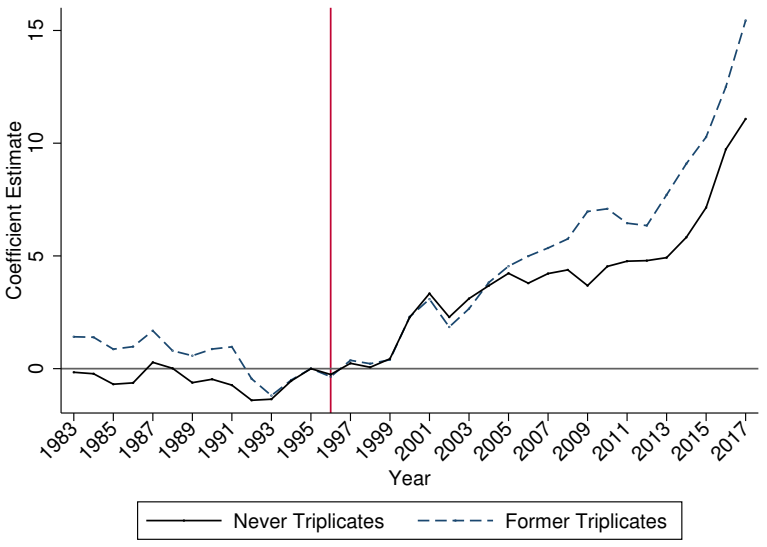

B: Drug Overdose Deaths

Notes: We estimate our primary event study using the triplicate states as controls and estimating effects separately for nevertriplicate states and former-triplicate states. State and year fixed effects included. The outcome in Panel A is OxyContin morphine equivalent doses per capita. The Panel B outcome is drug overdose deaths per 100,000.

\section{Figure 9: OxyContin Promotional Payments to Physicians}

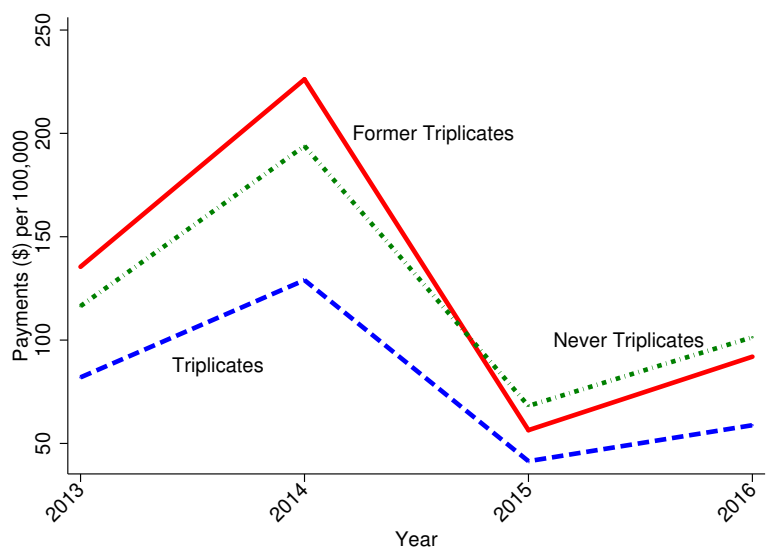

Notes: We used CMS Open Payments Data to calculate total payments and gifts made to physicians regarding OxyContin. We scaled this measure by population. The outcomes correspond to August 2013 - December 2016. Because the 2013 data only cover a partial year, we annualize the rate in that year. 


\section{Tables}

Table 1: Summary Statistics for 1991-1995

\begin{tabular}{|c|c|c|c|c|c|c|c|}
\hline Statistics for 1991-1995 & California & Idaho & Illinois & New York & Texas & Triplicate & Non-Triplicate \\
\hline \multicolumn{8}{|l|}{ Triplicate Program } \\
\hline First Year & 1939 & 1967 & 1961 & 1972 & 1982 & & \\
\hline Last Year & 2004 & 1997 & 2000 & 2001 & 1999 & & \\
\hline \multicolumn{8}{|l|}{ Annual Overdose Death Rates } \\
\hline Overdoses per 100,000 & 7.10 & 2.92 & 4.58 & 6.14 & 3.86 & 5.72 & 3.86 \\
\hline Overdose Rate Rank & 3 & 30 & 16 & 9 & 20 & - & - \\
\hline Overdoses (excluding cocaine) per 100,000 & 5.65 & 2.74 & 2.74 & 2.78 & 2.73 & 3.88 & 3.15 \\
\hline Overdose (excluding cocaine) Rate Rank & 4 & 23 & 21 & 20 & 24 & - & - \\
\hline Opioid Overdoses per 100,000 & 2.95 & 0.47 & 2.27 & 3.82 & 0.77 & 2.52 & 1.00 \\
\hline Opioid Overdose Rate Rank & 5 & 34 & 10 & 2 & 21 & - & - \\
\hline \multicolumn{8}{|l|}{ Demographics } \\
\hline$\%$ White, Non-Hispanic & $54.9 \%$ & $72.7 \%$ & $72.7 \%$ & $69.2 \%$ & $57.5 \%$ & $61.8 \%$ & $78.9 \%$ \\
\hline$\%$ Black, Non-Hispanic & $6.0 \%$ & $16.2 \%$ & $16.2 \%$ & $14.5 \%$ & $12.6 \%$ & $10.8 \%$ & $13.1 \%$ \\
\hline$\%$ Hispanic & $28.8 \%$ & $8.4 \%$ & $8.4 \%$ & $12.1 \%$ & $26.8 \%$ & $21.2 \%$ & $4.1 \%$ \\
\hline$\%$ Ages 25-44 & $34.1 \%$ & $32.3 \%$ & $32.3 \%$ & $32.4 \%$ & $32.8 \%$ & $33.1 \%$ & $31.8 \%$ \\
\hline$\%$ Ages $45-64$ & $17.6 \%$ & $19.2 \%$ & $19.1 \%$ & $20.0 \%$ & $17.7 \%$ & $18.4 \%$ & $19.6 \%$ \\
\hline$\%$ Ages $65+$ & $10.6 \%$ & $12.5 \%$ & $12.5 \%$ & $13.0 \%$ & $10.2 \%$ & $11.3 \%$ & $13.2 \%$ \\
\hline \% College Degree & $24.5 \%$ & $23.5 \%$ & $23.5 \%$ & $24.5 \%$ & $21.4 \%$ & $23.6 \%$ & $21.2 \%$ \\
\hline Population (in thousands) & 31,180 & 1,109 & 11,799 & 18,346 & 18,168 & 16,120 & 3,167 \\
\hline
\end{tabular}

Notes: All summary statistics are population-weighted means, except the population variable which is unweighted.

Table 2: Differences in Opioid Distribution (ARCOS)

\begin{tabular}{|c|c|c|c|c|c|c|}
\hline \multirow[t]{2}{*}{ Non-Triplicate $\mathrm{x}$} & \multicolumn{2}{|c|}{ OxyContin } & \multicolumn{2}{|c|}{ Oxycodone } & \multicolumn{2}{|c|}{ Hydrocodone } \\
\hline & (1) & (2) & (3) & (4) & $(5)$ & $(6)$ \\
\hline $1996-2000^{\dagger}$ & $0.707^{* * *}$ & $0.765^{* * *}$ & $0.637^{* * *}$ & 0.699* & -0.017 & -0.138 \\
\hline & {$[0.521,0.922]$} & {$[0.355,1.000]$} & {$[0.472,0.824]$} & {$[-0.061,1.512]$} & {$[-0.261,0.325]$} & {$[-0.813,0.199]$} \\
\hline $2001-2010$ & $0.615^{* * *}$ & $0.661^{* * *}$ & $2.259^{* * *}$ & $2.434^{* * *}$ & 0.052 & 0.005 \\
\hline & {$[0.309,0.991]$} & {$[0.321,0.994]$} & {$[1.198,3.443]$} & {$[1.413,3.641]$} & {$[-0.552,0.696]$} & {$[-0.794,0.427]$} \\
\hline $2011-2017^{\dagger}$ & $0.461^{* * *}$ & $0.517^{* *}$ & $2.949^{* * *}$ & $3.117^{* * *}$ & 0.101 & 0.077 \\
\hline & {$[0.222,0.776]$} & {$[0.150,0.845]$} & {$[0.979,4.532]$} & {$[1.347,4.738]$} & {$[-0.569,1.107]$} & {$[-0.638,0.648]$} \\
\hline Time-Varying Covariates & No & Yes & No & Yes & No & Yes \\
\hline $\mathrm{N}$ & 867 & 867 & 1,071 & 1,071 & 1,071 & 1,071 \\
\hline
\end{tabular}

Notes: ${ }^{* *}$ Significance $1 \%,{ }^{* *}$ Significance $5 \%,{ }^{*}$ Significance $10 \%$. The outcome is the per capita morphine equivalent doses of the substance listed. Estimated specifications include year fixed effects. The reported coefficients refer to the interaction of the given time period and an indicator for whether the state did not have a triplicate program in 1996. 95\% confidence intervals reported in brackets are estimated by wild bootstrap. Regressions are population-weighted. Time-varying covariates include the fraction non-Hispanic white, fraction non-Hispanic black, fraction Hispanic, log of population, fraction with college degree, fraction ages 25-44, fraction ages 45-64, and fraction ages 65+.

$\dagger$ OxyContin data only cover 2000-2016 and, therefore, the 1997-2000 category only refers to 2000; the 2011-2017 category refers to 2011-2016. 
Table 3: Difference-in-Differences Estimates: Drug Overdose Death Rate

\begin{tabular}{ccccc}
\hline & \multicolumn{4}{c}{ A: Overdose Deaths per 100,000} \\
Non-Triplicate $\times$ & $(1)$ & $(2)$ & $(3)$ & $(4)$ \\
\hline $1996-2000$ & $1.244^{* *}$ & $1.323^{* * *}$ & $1.125^{* *}$ & $1.452^{* *}$ \\
& {$[0.443,2.463]$} & {$[0.453,2.533]$} & {$[0.045,2.705]$} & {$[0.297,2.844]$} \\
$2001-2010$ & $3.758^{* *}$ & $4.566^{* * *}$ & $4.221^{* *}$ & $4.445^{* * *}$ \\
& {$[1.612,6.399]$} & {$[2.206,6.600]$} & {$[1.344,6.953]$} & {$[2.557,6.245]$} \\
$2011-2017$ & $6.248^{* *}$ & $7.903^{* * *}$ & $6.944^{* * *}$ & $6.816^{* * *}$ \\
& {$[3.012,9.486]$} & {$[4.136,10.496]$} & {$[4.429,8.886]$} & {$[4.278,9.014]$} \\
Joint P-Value & 0.016 & 0.000 & 0.002 & 0.001 \\
Weighted & No & Yes & Yes & Yes \\
Covariates & No & No & Yes & Yes \\
Region-Time Dummies & No & No & No & Yes \\
Mean 1991-1995 & 3.874 & 4.436 & 4.436 & 4.436 \\
N & 1,377 & 1,377 & 1,377 & 1,377 \\
\hline & & B: Opioid Overdose & Deaths per 100,000 & \\
Non-Triplicate & $(5)$ & $(6)$ & $(7)$ & $(8)$ \\
\hline 1996-2000 & $0.702^{* *}$ & $0.631^{* *}$ & $0.821^{*}$ & $1.044^{* *}$ \\
& {$[0.136,1.648]$} & {$[0.112,1.672]$} & {$[-0.154,1.892]$} & {$[0.003,2.075]$} \\
2001-2010 & $2.675^{* *}$ & $2.993^{* * *}$ & $2.766^{* *}$ & $3.011^{* * *}$ \\
& {$[1.130,4.527]$} & {$[1.195,4.384]$} & {$[0.042,4.918]$} & {$[0.988,4.932]$} \\
2011-2017 & $5.133^{* *}$ & $5.946^{* * *}$ & $4.789^{* * *}$ & $4.543^{* * *}$ \\
& {$[1.551,8.417]$} & {$[1.805,8.919]$} & {$[1.893,6.879]$} & {$[1.830,6.645]$} \\
Joint P-Value & 0.038 & 0.011 & 0.018 & 0.013 \\
Weighted & No & Yes & Yes & Yes \\
Covariates & No & No & Yes & Yes \\
Region-Time Dummies & No & No & No & Yes \\
Mean 1991-1995 & 1.173 & 1.476 & 1.476 & 1.476 \\
N & 1,377 & 1,377 & 1,377 & 1,377 \\
\hline
\end{tabular}

Notes: ${ }^{* * *}$ Significance $1 \%,{ }^{* *}$ Significance $5 \%,{ }^{*}$ Significance $10 \%$. Outcome is overdose deaths or opioid overdose deaths per 100,000. The reported coefficients refer to the interaction of the given time period and an indicator for whether the state did not have a triplicate program in 1996. Estimates are relative to pre-period 1991-1995. 95\% confidence intervals reported in brackets are estimated by wild bootstrap. All models include state and year fixed effects. Covariates include the fraction non-Hispanic white, fraction non-Hispanic black, fraction Hispanic, log of population, fraction with college degree, fraction ages $25-44$, fraction ages 45-64, and fraction ages 65+. "Joint P-Value" refers to the p-value from a joint hypothesis test that all three non-triplicate post effects are equal to zero and is also estimated using a restricted wild bootstrap. 


\section{Table 4: Robustness Tests}

\begin{tabular}{|c|c|c|c|c|}
\hline \multirow[b]{2}{*}{ Non-Triplicate $\times$} & \multicolumn{4}{|c|}{ Overdose Deaths per 100,000} \\
\hline & $\begin{array}{l}\text { Baseline } \\
\text { Results }\end{array}$ & $\begin{array}{c}\text { Select on } \\
\text { Population Size }\end{array}$ & $\begin{array}{l}\text { Select on PDMP } \\
\text { States in } 1996\end{array}$ & $\begin{array}{c}\text { Control for } \\
\text { Policy Variables }\end{array}$ \\
\hline & (1) & $(2)$ & (3) & (4) \\
\hline 1996-2000 & $1.125^{* *}$ & $4.104^{* *}$ & $1.831^{*}$ & $1.557^{* *}$ \\
\hline & {$[0.045,2.705]$} & {$[1.444,6.443]$} & {$[-0.165,3.826]$} & {$[0.345,2.714]$} \\
\hline $2001-2010$ & $4.221^{* *}$ & $8.204^{* * *}$ & $6.137^{* * *}$ & $5.448^{* * *}$ \\
\hline & {$[1.344,6.953]$} & {$[4.874,11.118]$} & {$[2.972,9.303]$} & {$[2.913,7.372]$} \\
\hline 2011-2017 & $6.944 * * *$ & $10.487^{* *}$ & $10.698 * * *$ & $8.811^{* * *}$ \\
\hline & {$[4.429,8.886]$} & {$[0.759,19.929]$} & {$[7.607,13.789]$} & {$[5.863,11.689]$} \\
\hline Joint P-Value & 0.002 & 0.012 & 0.001 & 0.000 \\
\hline Mean 1991-1995 & 4.436 & 5.134 & 5.346 & 4.436 \\
\hline $\mathrm{N}$ & 1,377 & 216 & 405 & 1,377 \\
\hline
\end{tabular}

Notes: ***Significance 1\%, **Significance $5 \%,{ }^{*}$ Significance $10 \%$. Outcome is overdose deaths per 100,000 . The reported coefficients refer to the interaction of the given time period and an indicator for whether the state did not have a triplicate program in 1996. Estimates are relative to pre-period 1991-1995. 95\% confidence intervals reported in brackets are estimated by wild bootstrap. All models include state and year fixed effects and time-varying covariates (see Table 3 for details). Column (1) repeats the Column (3) results from Table 3. Column (2) selects on the four non-triplicate states with the largest populations in 1990 along with the four largest triplicate states. Column (3) selects on states with some form of PDMP (triplicate, duplicate, electronic) in 1996. Column (4) includes policy controls for PDMPs (any PDMP and electronic PDMP), "must access" PDMPs, pain clinic regulation, medical marijuana laws, and operational/legal medical marijuana dispensaries. "Joint P-Value" refers to the p-value from a joint hypothesis test that all three non-triplicate post effects are equal to zero and is also estimated using a restricted wild bootstrap. 


\section{Appendix A}

\section{Appendix Figures}

Figure A1: Example of Purdue Pharma Focus Group Recommendations

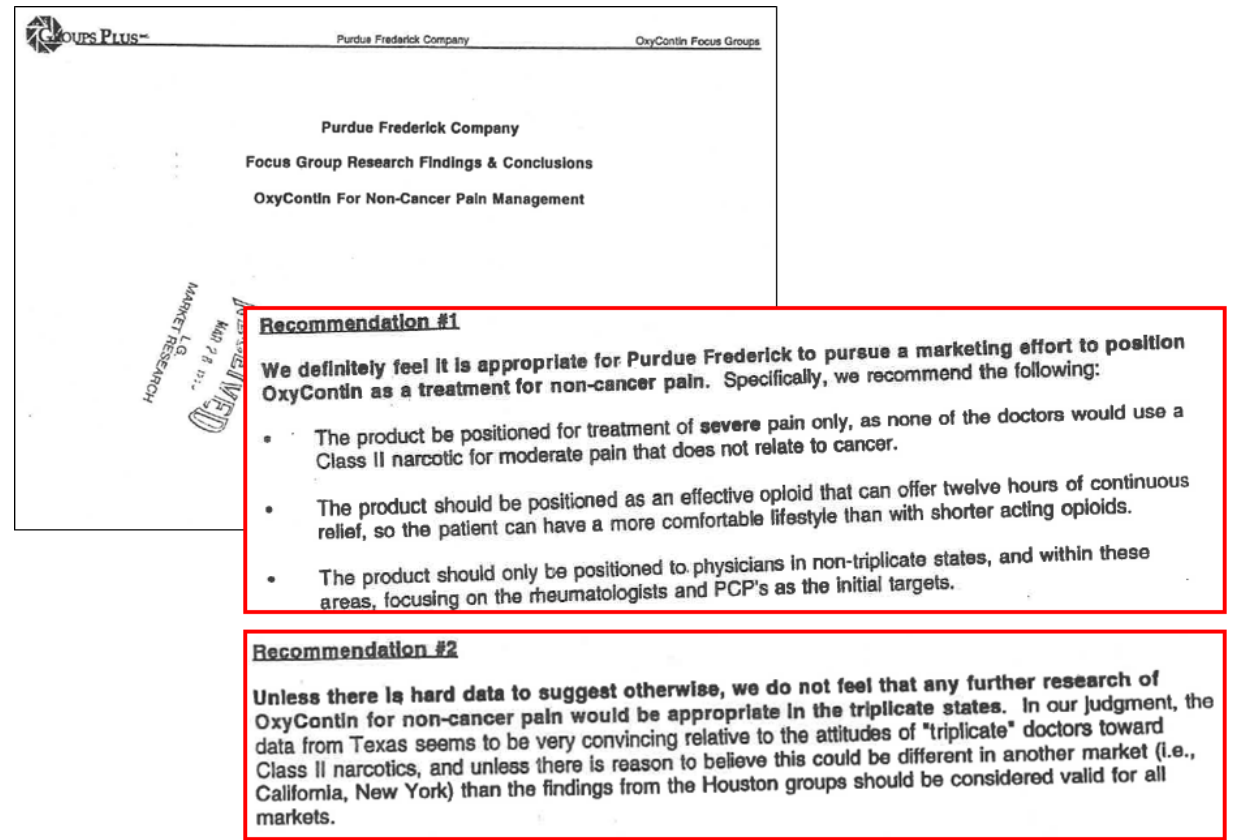


Figure A2: ICD Code Change in 1999

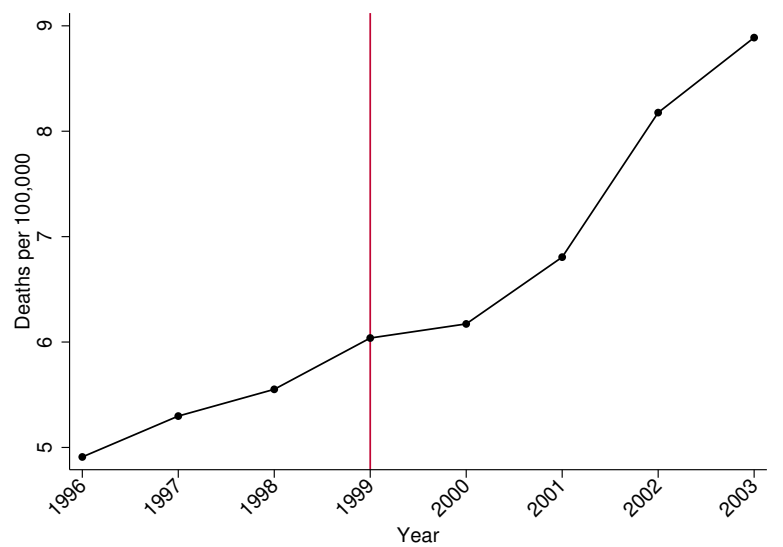

A: Drug Overdose Deaths per 100,000

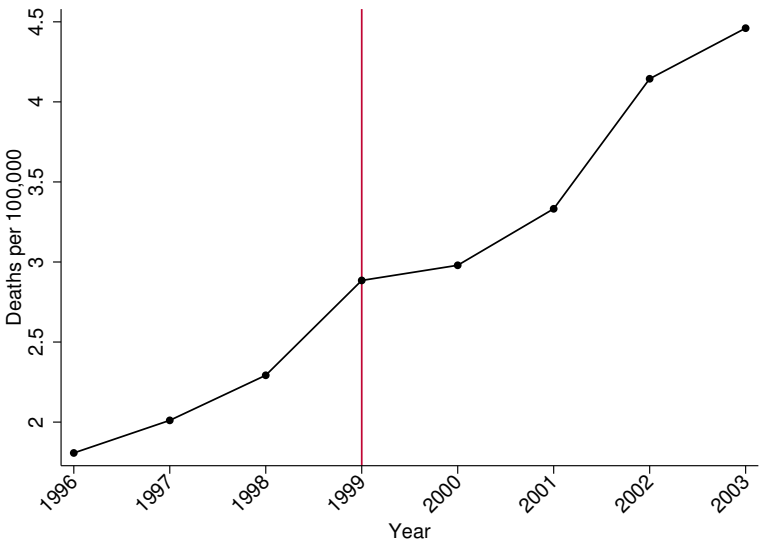

B: Opioid Overdose Deaths per 100,000

Notes: We use geocoded NVSS data to construct overdose deaths per 100,000. These figures study the transition from ICD-9 to ICD-10 codes in 1999 . 
Figure A3: OxyContin Adoption by State

A. Medicaid OxyContin Prescriptions per 1,000 Benes in 1996

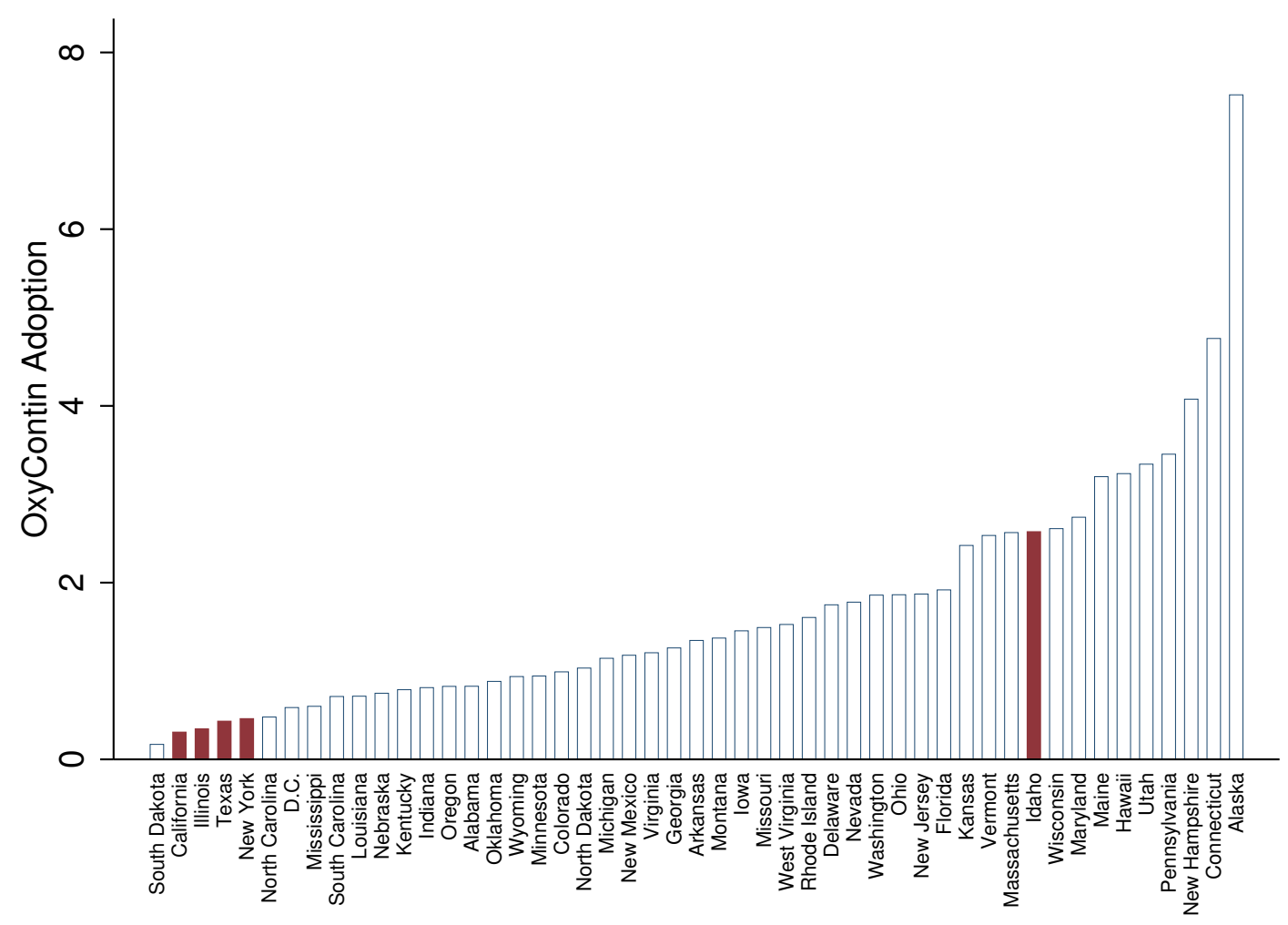

B. ARCOS Per Capita OxyContin Morphine Equivalent Doses in 2000

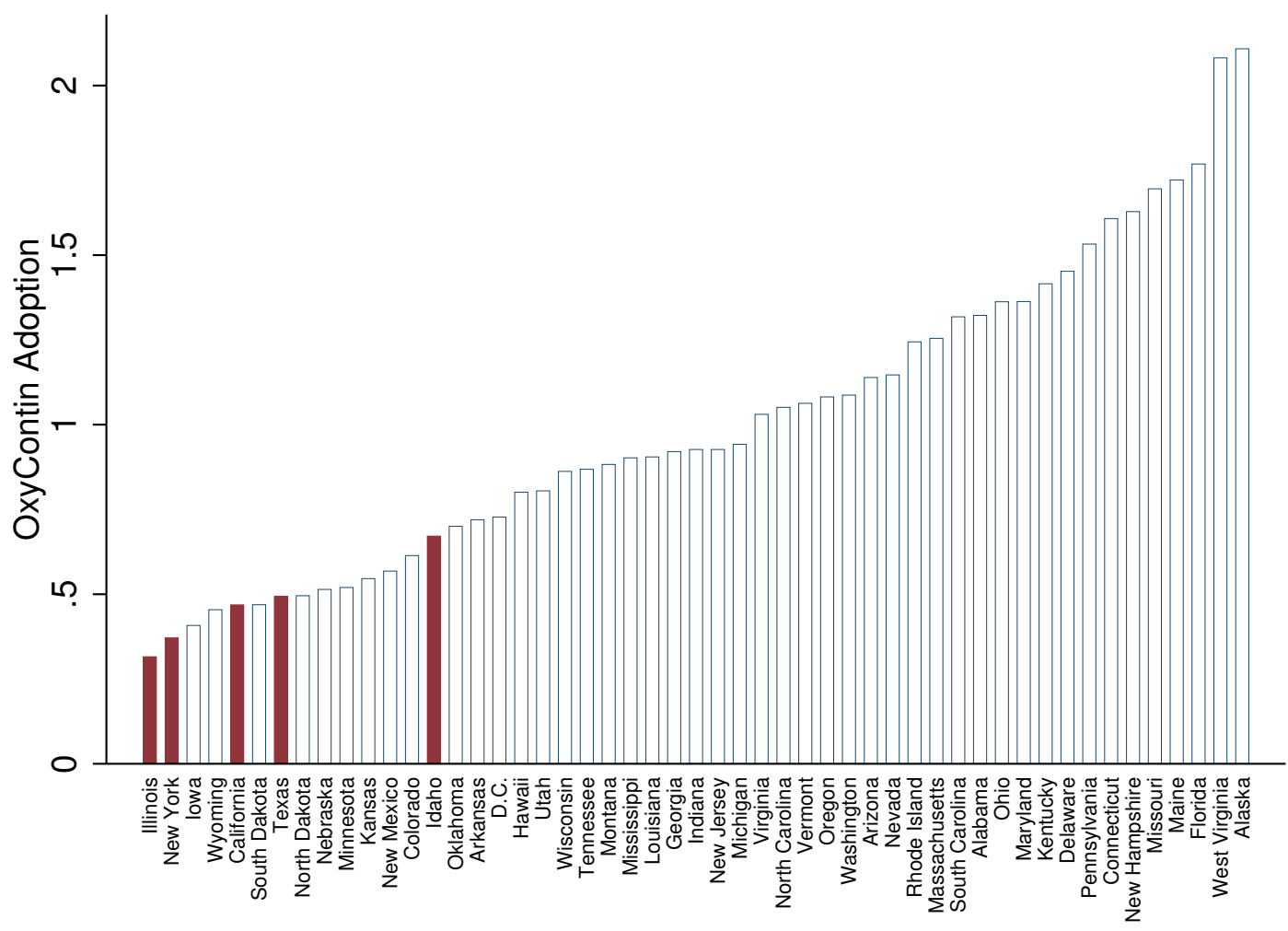


Figure A4: Overdose Death Rate Event Studies

\section{Unweighted}

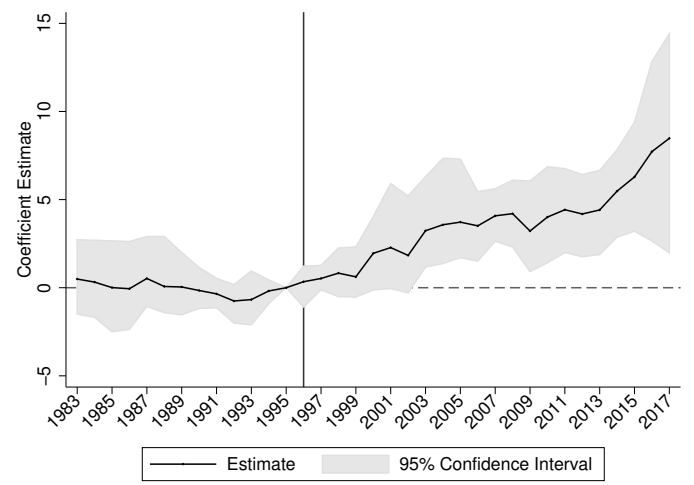

A: Drug Overdoses per 100,000

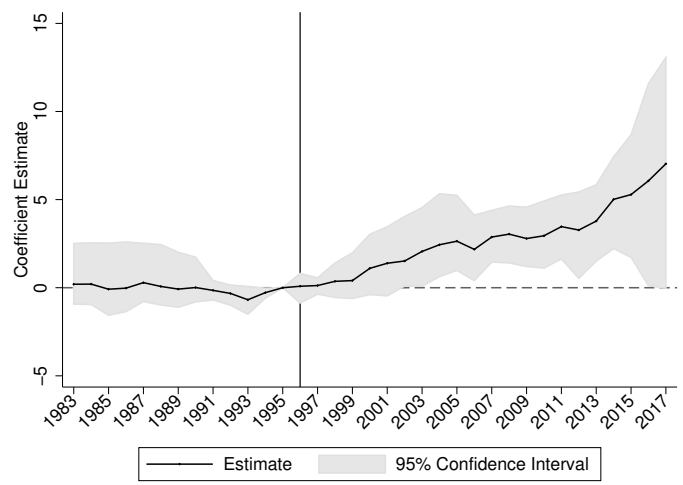

B: Opioid Overdoses per 100,000

\section{Unweighted, With Covariates}

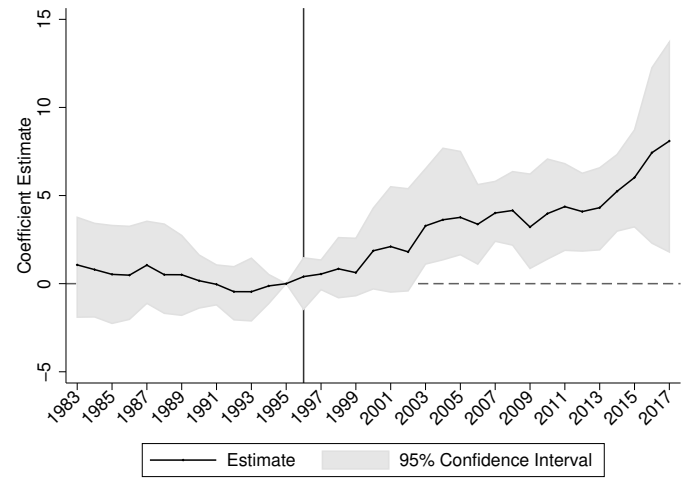

C: Drug Overdoses per 100,000

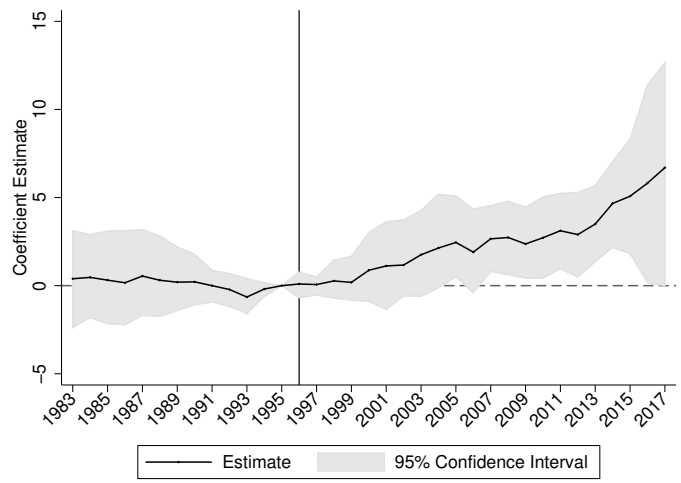

D: Opioid Overdoses per 100,000

\section{Weighted, With Covariates}

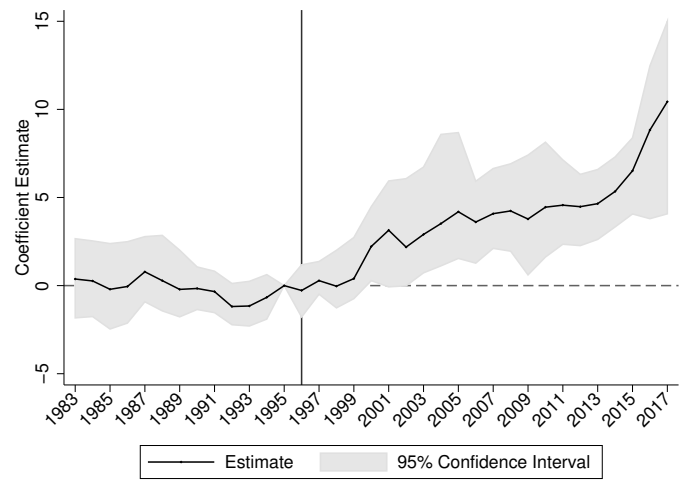

E: Drug Overdoses per 100,000

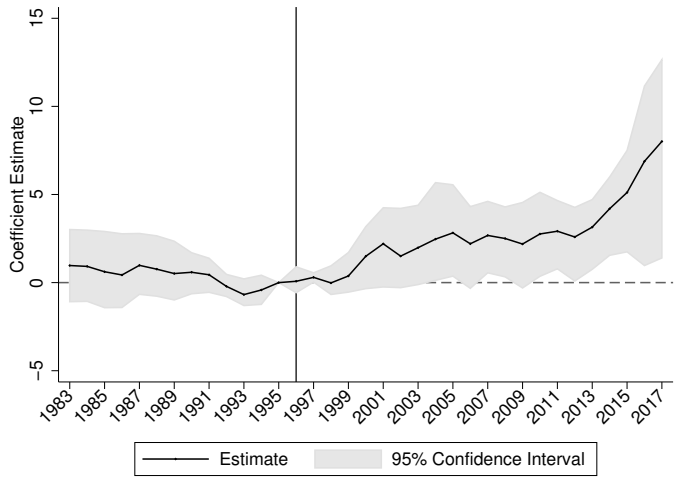

F: Opioid Overdoses per 100,000

Notes: We use geocoded NVSS data to construct overdose and opioid overdose deaths per 100,000. See text for exact ICD codes used in each period. 95\% confidence intervals are generated using a clustered (at state) wild bootstrap. Estimates are normalized to 0 in 1995. All models include state and year fixed effects. When covariates are specified, the models include the fraction non-Hispanic white, fraction non-Hispanic black, fraction Hispanic, log of population, fraction with college degree, fraction ages 25-44, fraction ages 45-64, and fraction ages 65+. Panels $\mathrm{E}$ and $\mathrm{F}$ are population-weighted; the others are not. 
Figure A5: Drug Overdose Death Rate Changes: Triplicate States vs. Bordering States (2008-2017 Relative to 1986-1995)
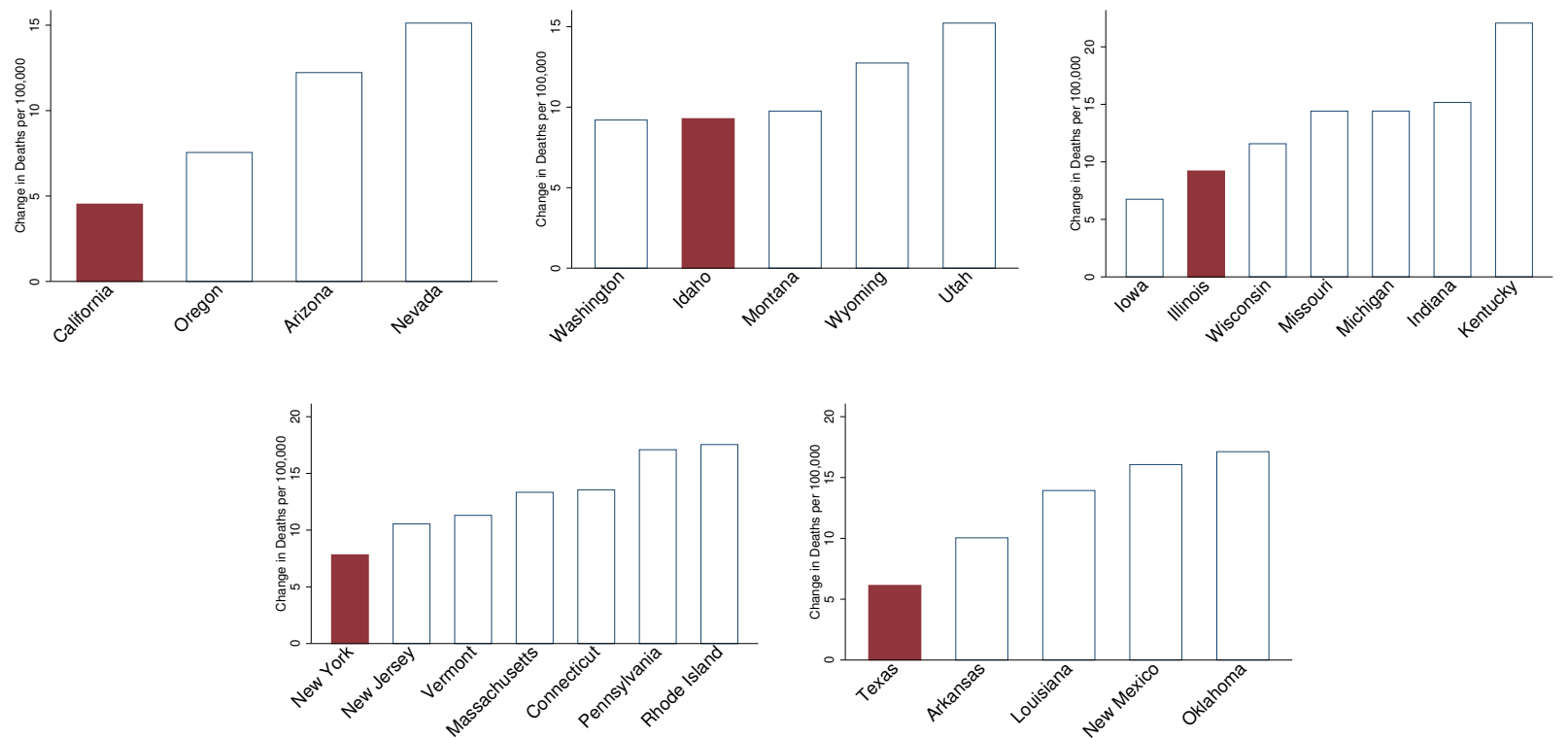

Notes: We construct the change in overdose deaths per 100,000 for 2008-2017 relative to 1986-1995. We plot this change for each triplicate state relative to its bordering states. 
Figure A6: Overdose Death Rate Differences by Type of Opioid for 1999-2017

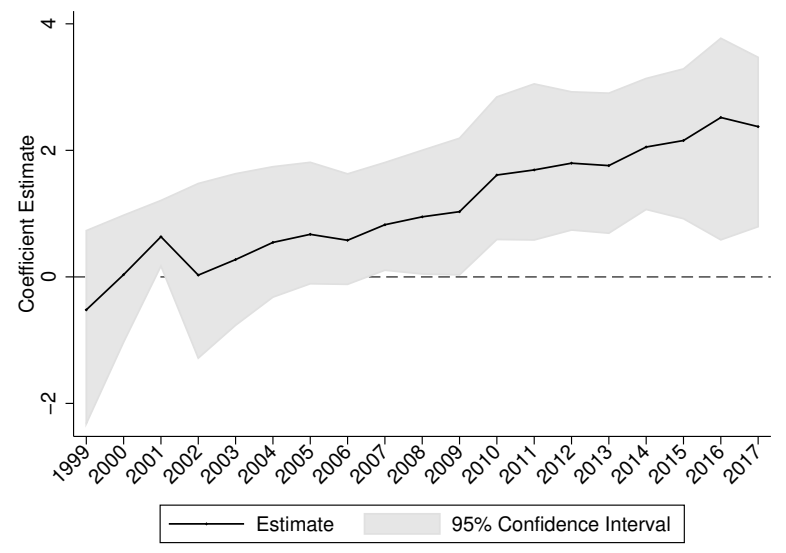

A: Natural and Semisynthetic Opioids

$(\mathrm{T} 40.2)$

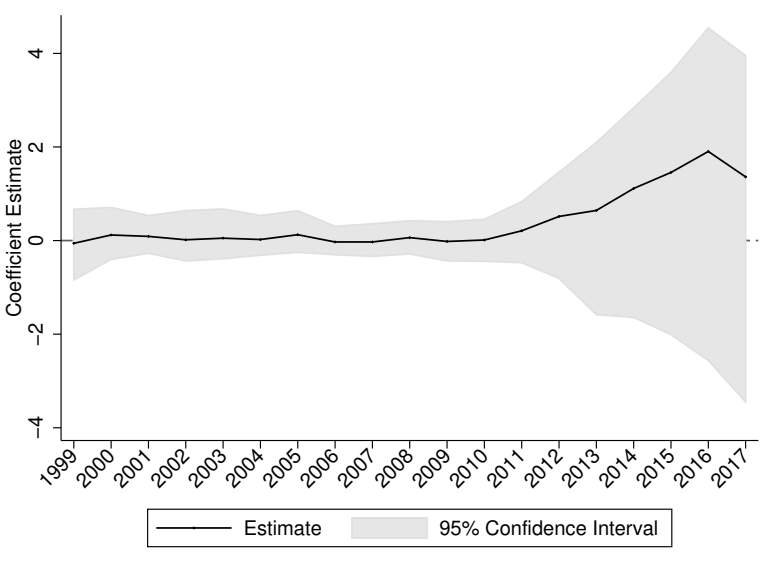

B: Heroin (T40.1)

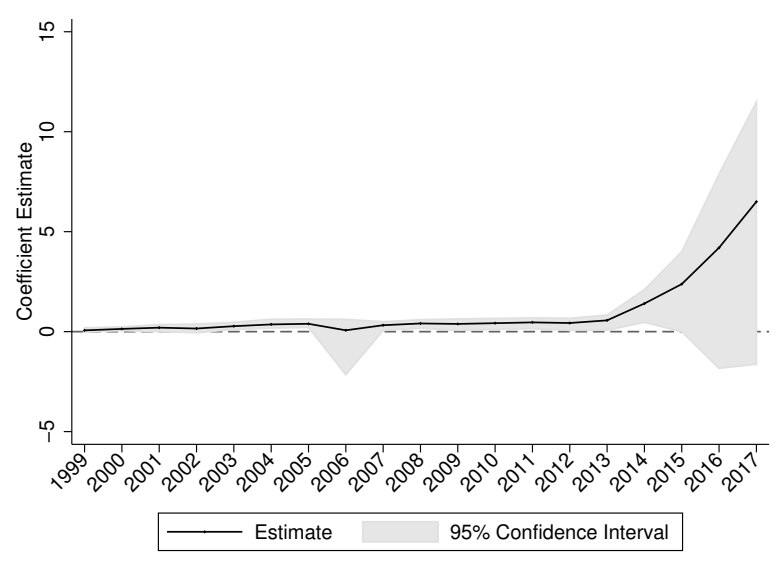

\section{C: Synthetic Opioids (T40.4)}

Notes: We use geocoded NVSS data to construct overdose deaths per 100,000 for the reported opioid types (see text for additional information). We show estimates from a regression which includes year fixed effects and non-triplicate indicators interacted with year estimates. 95\% confidence intervals are generated using a clustered (at state) wild bootstrap. 
Figure A7: Opioid-Related Substance Abuse Treatment Admissions

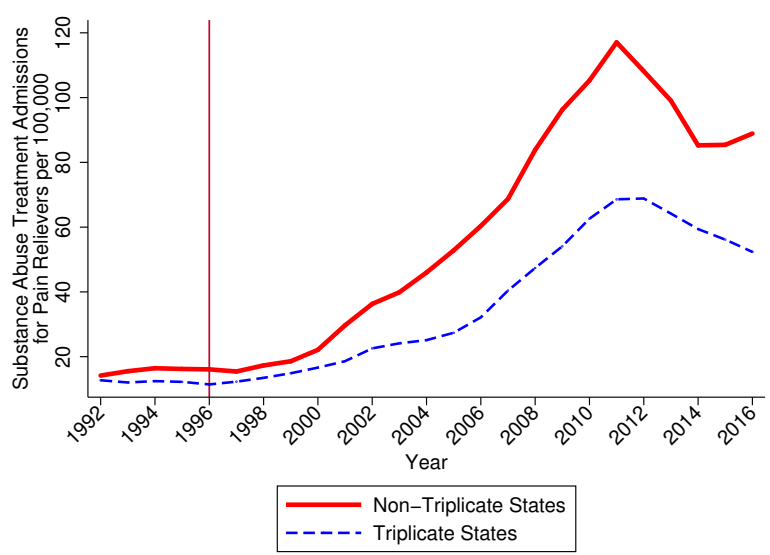

\section{Pain Reliever Substance Abuse Treatment}

Admissions per 100,000

Notes: Substance abuse treatment admissions are calculated using the Treatment Episode Data Set. 
Figure A8: Drug Overdose Death Rate Changes Sorted by Population Size: 1996-2005 Relative to 1986-1995

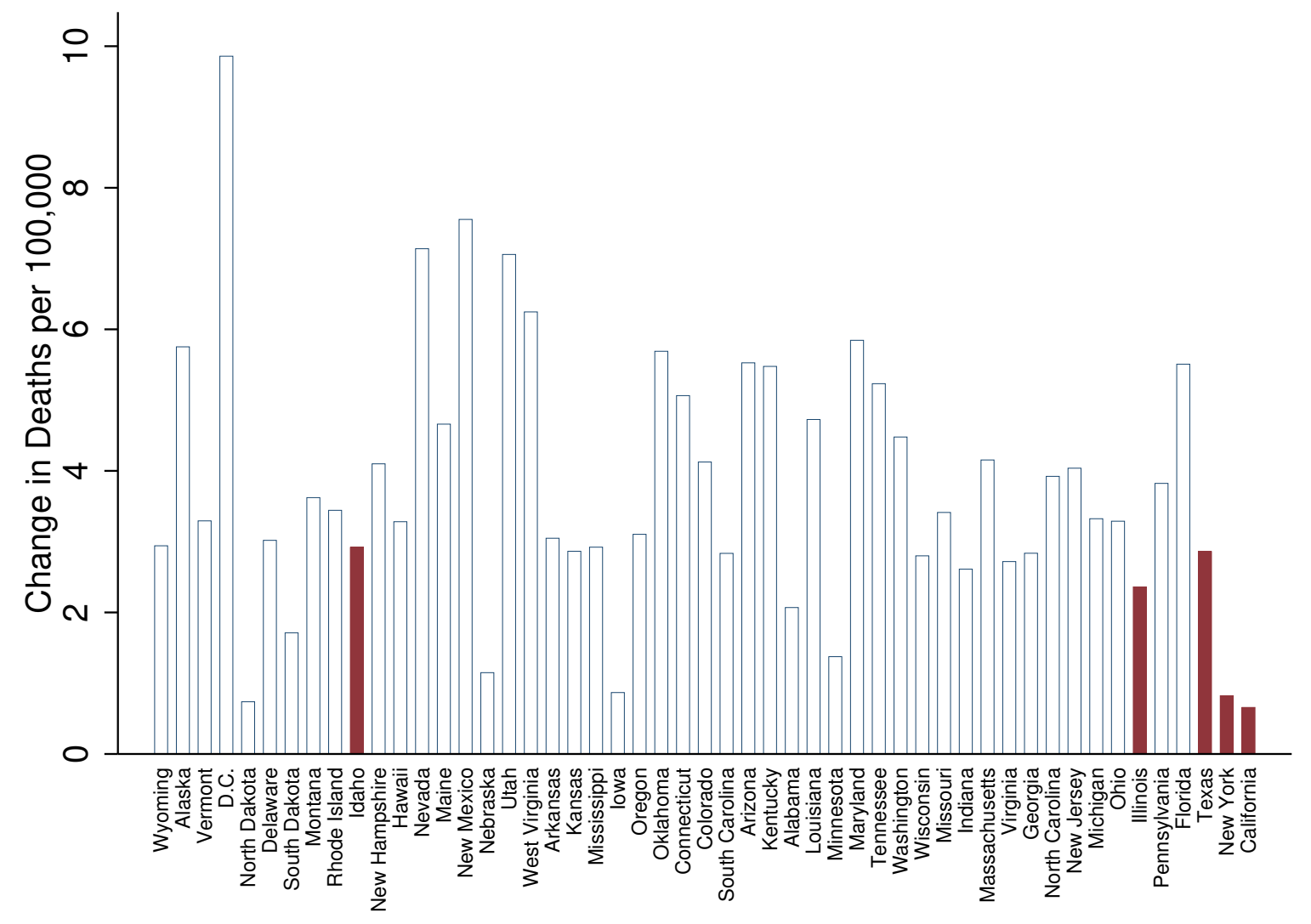

Notes: We construct the change in overdose deaths per 100,000 for 1996-2005 relative to 1986-1995. States are sorted by population size from lowest to highest. 
Figure A9: County-Level Overdose Death Rate Event Studies By Metropolitan Area Size

\section{Overdose Deaths per 100,000}

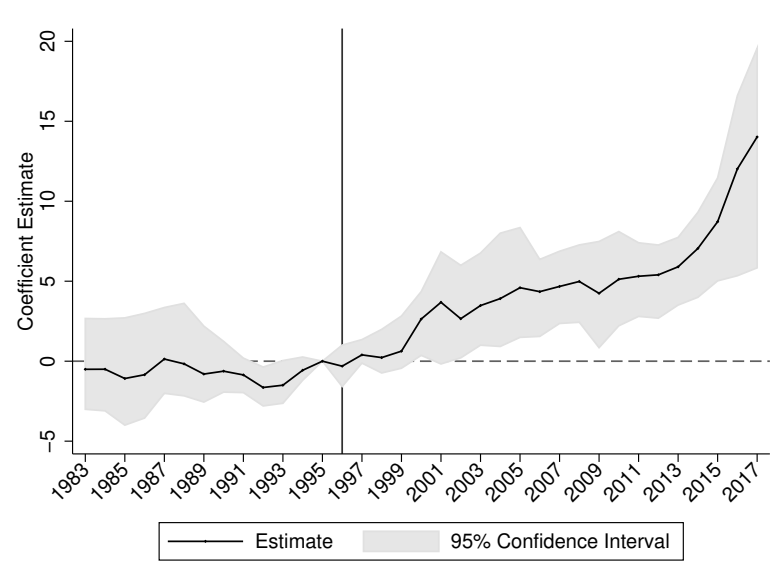

A: Counties of metro areas

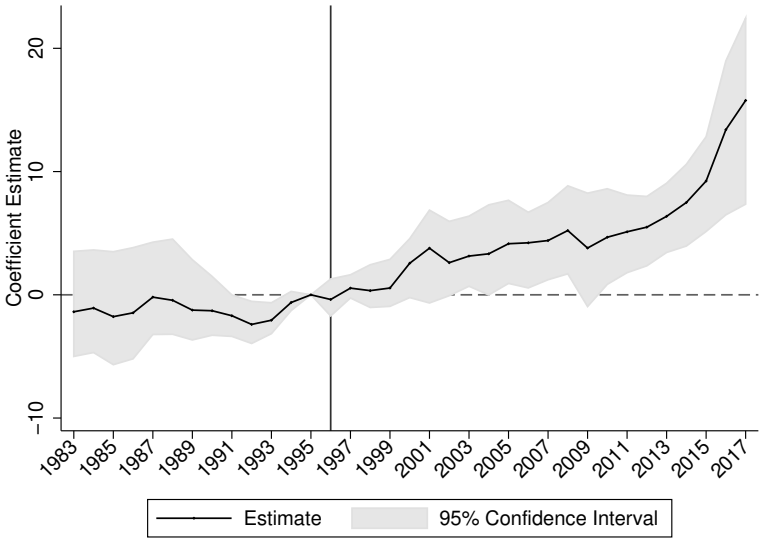

B: Central counties of metro areas of 1 million population or more

Notes: The outcome is county-level overdose deaths per 100,000. 95\% confidence intervals are generated using a clustered (at state) wild bootstrap. Estimates are normalized to 0 in 1995. Counties are categorized by the United States Department of Agriculture's Economic Research Service in 1993. We estimate the main event study specification at the county-level. County and year fixed effects included in all models. $N=28,910$ (826 counties) for Panel A; $N=6,125$ (175 counties) for Panel B. 
Figure A10: Comparing PDMP Strength by Triplicate State Status

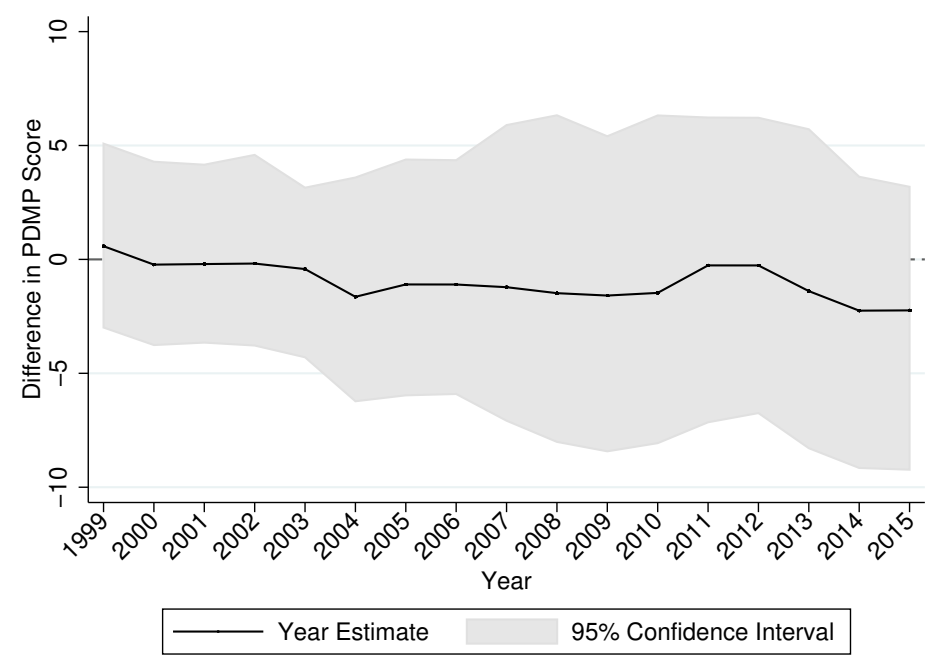

Notes: Each estimate represents the cross-sectional difference in the outcome variable, comparing non-triplicate states relative to triplicate states, for the available years of the index (1999-2015). The outcome is the Pardo (2017) index of PDMP strength. 95\% confidence intervals generated using wild bootstrap clustered by state. We select on states with any type of PDMP in 1996. 


\section{Figure A11: Differences in Other Deaths of Despair by Triplicate State Status}

\section{Main Estimates}

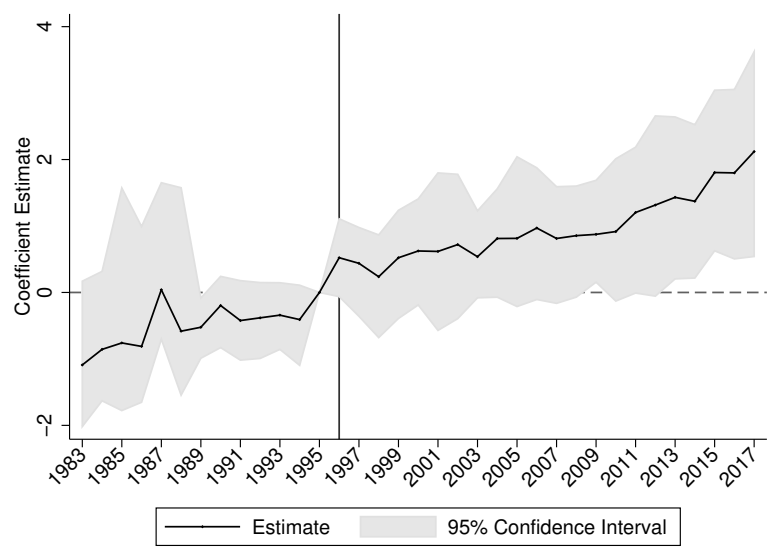

A: Suicides

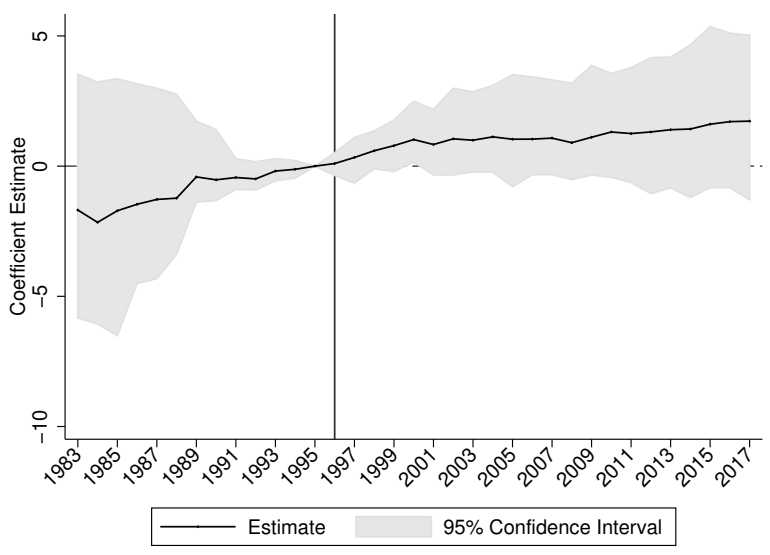

B: Alcohol-Related Liver Diseases

Detrended

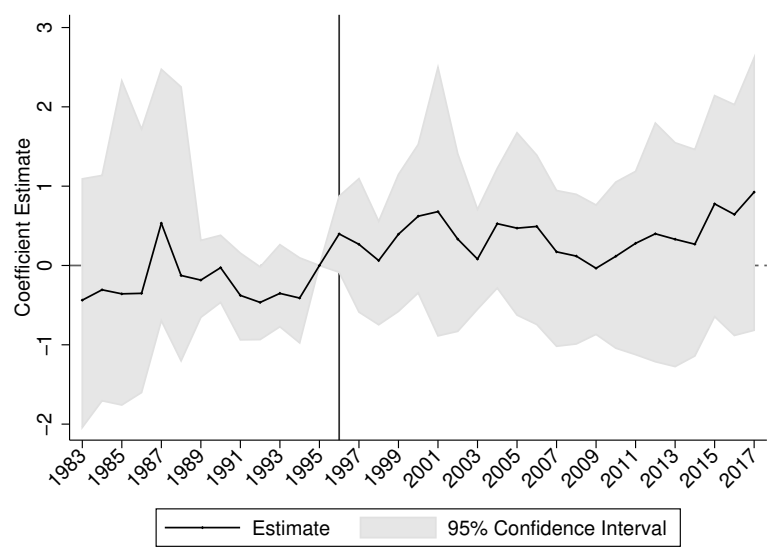

C: Suicides

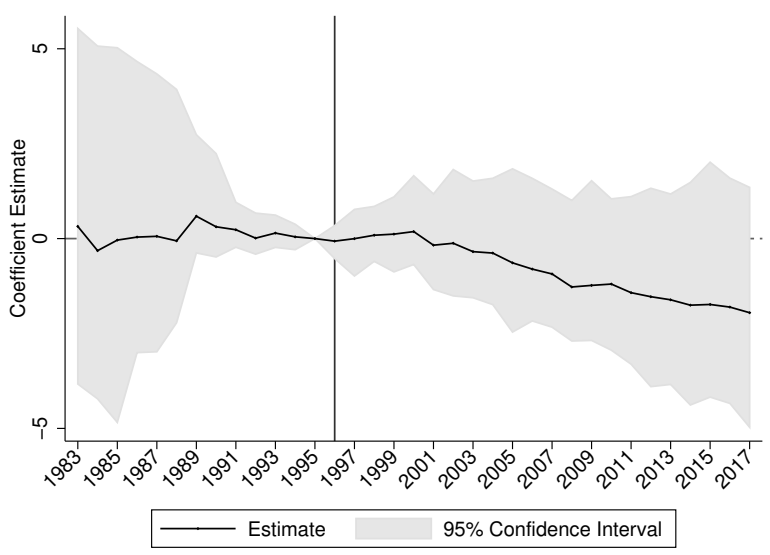

D: Alcohol-Related Liver Diseases

Notes: We use geocoded NVSS data to construct suicides (excluding those involving overdoses) and alcohol-related liver disease deaths per 100,000. These figures report event study estimates from a population-weighted regression which includes state and year fixed effects. 95\% confidence intervals are generated using a clustered (at state) wild bootstrap. Estimates are normalized to 0 in 1995. In Panel C and D, we show estimates after detrending. We detrend by first estimating a model with state fixed effects, year fixed effects, and a linear time trends interacted with non-triplicate status. This model is estimated using only pre-1996 data. We then subtract off the value of the trend term multiplied by the estimated coefficient for the entire period. This detrended variable is used as the outcome to estimate the event study. 
Figure A12: Event Study: Comparing States with Low Initial Oxycodone Prescribing

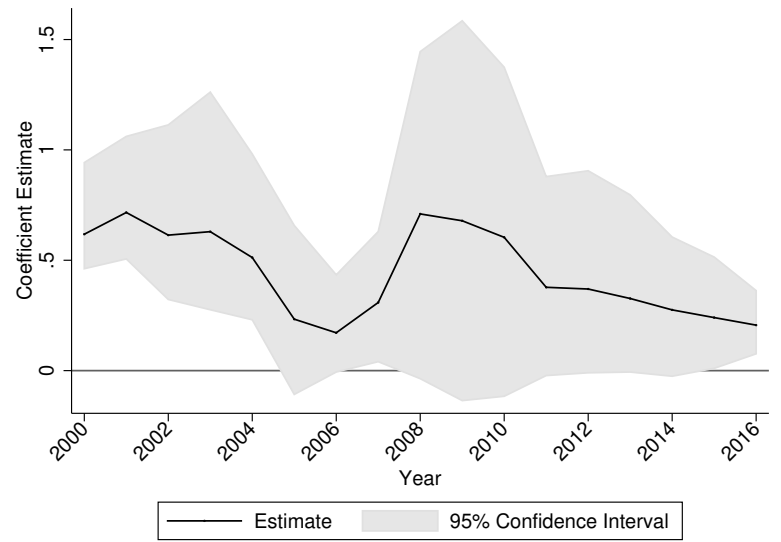

A: OxyContin Distribution

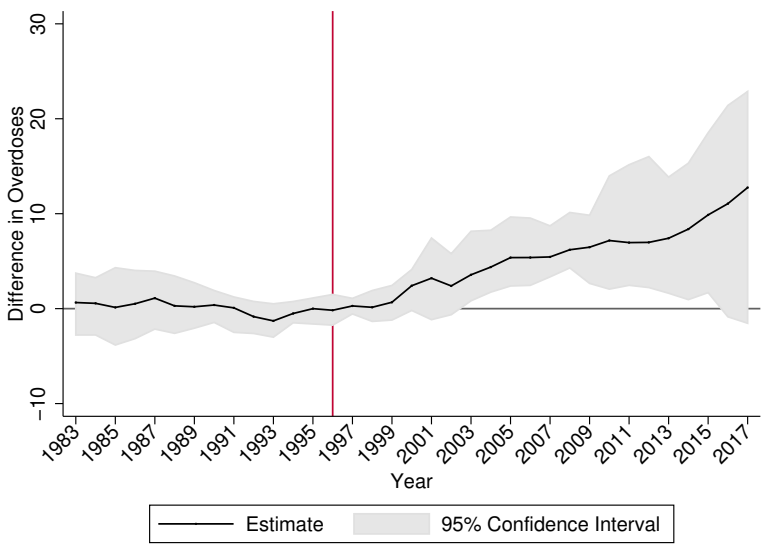

B: Overdose Deaths per 100,000

Notes: We compare "low oxycodone" non-triplicate states to triplicate states. "Low oxycodone" states are defined as in terms of oxycodone Medicaid prescriptions per 1,000 beneficiaries in 1991-1995. Figure A relies on ARCOS data for 2000-2016. Figure B uses mortality data for 1983-2017. All specifications include state and time fixed effects. Confidence intervals are generated by a wild bootstrap. 
Figure A13: Median OxyContin Supply for Never-Triplicates, Former-Triplicates, and 1996 Triplicates

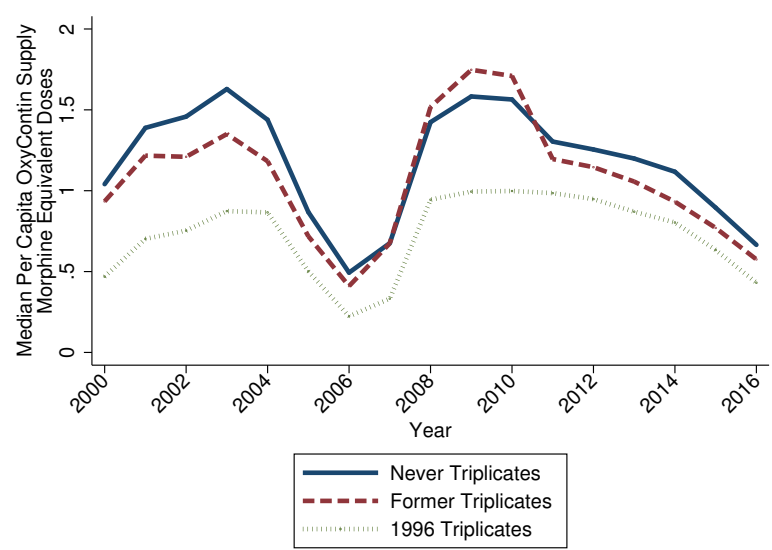

Notes: We calculate the median OxyContin distribution, measured in morphine equivalent doses, using the ARCOS data by former and 1996 triplicate status. 
Figure A14: OxyContin Promotional Payments to Physicians - Scaled by Total Size of Payments in State

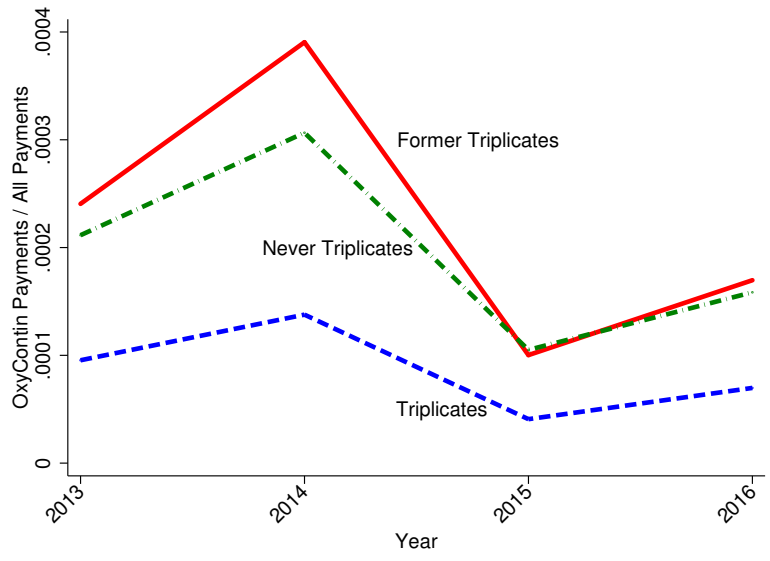

Notes: We used CMS Open Payments Data to calculate total payments and gifts made to physicians regarding OxyContin. We scaled this measure by total payments and gifts made to physicians across all drugs. The outcomes correspond to August 2013 - December 2016.

Figure A15: Event Study: Effects of Triplicate Repeal

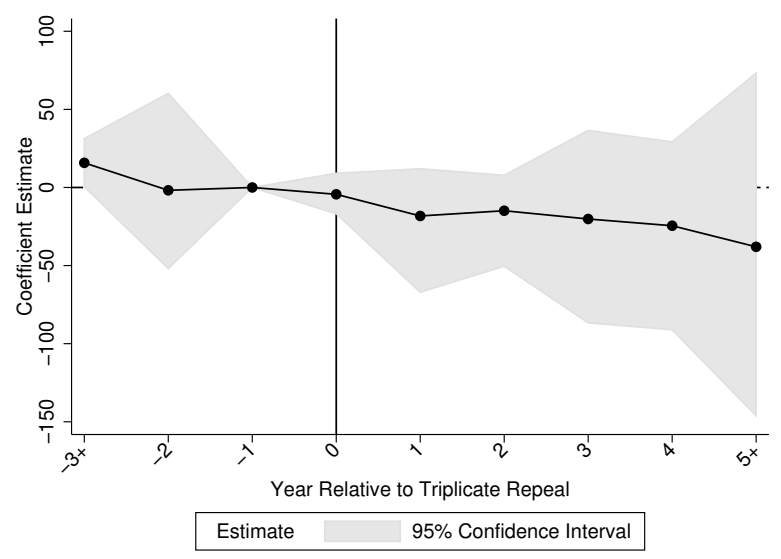

A: OxyContin Prescriptions

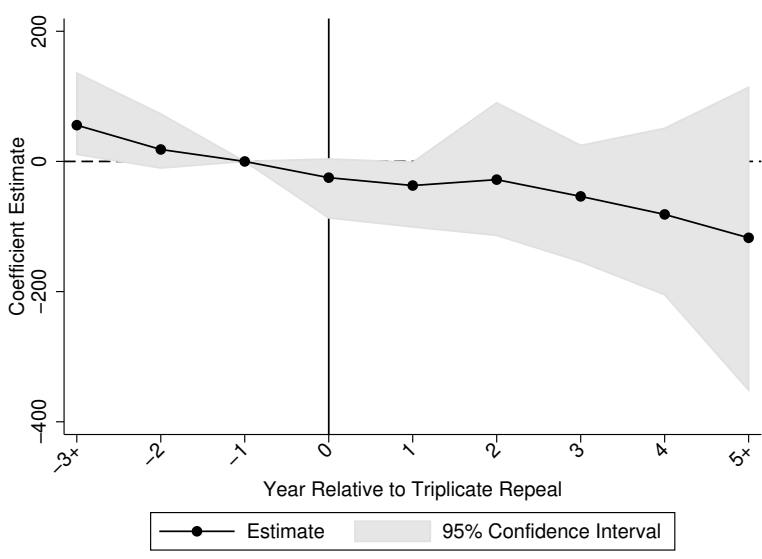

B: Oxycodone Prescriptions

Notes: We study Medicaid OxyContin and oxycodone prescriptions per 1,000 beneficiaries. We exclude the former triplicate states since they repealed prior to OxyContin's introduction. The sample period for OxyContin prescriptions is 1996-2005; the sample for oxycodone prescriptions is 1991-2005. We include state and time fixed effects in addition to the time-relative-to-event indicators. Confidence intervals are generated by a wild bootstrap. 


\section{Appendix Tables}

Table A1: Robustness Tests: Opioid-Related Overdose Deaths per 100,000

\begin{tabular}{ccccc}
\hline Non-Triplicate $\times$ & $\begin{array}{c}\text { Baseline } \\
\text { Results }\end{array}$ & $\begin{array}{c}\text { Select on } \\
\text { Population Size }\end{array}$ & $\begin{array}{c}\text { Select on PDMP } \\
\text { States in 1996 }\end{array}$ & $\begin{array}{c}\text { Control for } \\
\text { Policy Variables }\end{array}$ \\
\hline \multirow{2}{*}{$1996-2000$} & $(1)$ & $(2)$ & $(3)$ & $(4)$ \\
& $0.821^{*}$ & $2.880^{* *}$ & 1.032 & $1.297^{*}$ \\
$2001-2010$ & {$[-0.154,1.892]$} & {$[0.973,5.496]$} & {$[-0.802,2.867]$} & {$[-0.235,2.729]$} \\
& $2.766^{* *}$ & $5.798^{* * *}$ & $4.374^{* * *}$ & $3.396^{*}$ \\
$2011-2017$ & {$[0.042,4.918]$} & {$[2.632,8.877]$} & {$[1.311,7.436]$} & {$[-0.255,5.661]$} \\
& $4.789^{* * *}$ & $6.946^{*}$ & $7.590^{* * *}$ & $5.712^{* * *}$ \\
Joint P-Value & {$[1.893,6.879]$} & {$[-1.036,14.747]$} & {$[4.533,10.647]$} & {$[1.962,8.192]$} \\
Mean 1991-1995 & 0.018 & 0.042 & 0.009 & 0.023 \\
$\mathrm{~N}$ & 1.476 & 1.881 & 2.050 & 1.476 \\
& 1,377 & 216 & 405 & 1,377 \\
\hline
\end{tabular}

Notes: ***Significance 1\%, **Significance $5 \%$, *Significance 10\%. Outcome is opioid overdose deaths per 100,000. The reported coefficients refer to the interaction of the given time period and an indicator for whether the state did not have a triplicate program in 1996. Estimates are relative to pre-period 1991-1995. 95\% confidence intervals reported in brackets are estimated by wild bootstrap. All models include state and year fixed effects and time-varying covariates (see Table 3 for details). Column (1) repeats the column 3 results from Table 3. Column (2) selects on the four non-triplicate states with the largest populations in 1990 along with the four largest triplicate states. Column (3) selects on states with some form of PDMP (triplicate, duplicate, electronic) in 1996. Column (4) includes policy controls for PDMPs (any PDMP and electronic PDMP), "must access" PDMPs, pain clinic regulation, medical marijuana laws, and operational/legal medical marijuana dispensaries. "Joint P-Value" refers to the p-value from a joint hypothesis test that all three non-triplicate post effects are equal to zero and is also estimated using a restricted wild bootstrap. 
Table A2: Initial State Oxycodone Prescribing Prevalence, 1991-1995

\begin{tabular}{|c|c|}
\hline State & $\begin{array}{c}\text { Medicaid Prescriptions per } 1,000 \text { Benes } \\
(1991-1995)\end{array}$ \\
\hline Texas & 1.684214 \\
\hline Illinois & 2.726036 \\
\hline California & 7.61223 \\
\hline Kentucky & 8.034977 \\
\hline Michigan & 10.25393 \\
\hline New York & 11.24532 \\
\hline Idaho & 19.17949 \\
\hline Indiana & 21.00094 \\
\hline Washington & 21.43061 \\
\hline South Dakota & 22.43416 \\
\hline Rhode Island & 23.02265 \\
\hline Arkansas & 25.87149 \\
\hline Minnesota & 26.95233 \\
\hline Mississippi & 27.55857 \\
\hline Iowa & 30.33542 \\
\hline Oklahoma & 30.39548 \\
\hline North Dakota & 30.90121 \\
\hline Nebraska & 34.75153 \\
\hline Tennessee & 36.05626 \\
\hline Alabama & 36.32855 \\
\hline South Carolina & 38.62302 \\
\hline District Of Columbia & 39.77379 \\
\hline Kansas & 40.51961 \\
\hline Georgia & 40.60681 \\
\hline Missouri & 41.19653 \\
\hline West Virginia & 42.2592 \\
\hline Oregon & 43.85831 \\
\hline Florida & 44.15401 \\
\hline North Carolina & 44.5726 \\
\hline Louisiana & 45.27365 \\
\hline Ohio & 45.36346 \\
\hline Wyoming & 52.09373 \\
\hline Wisconsin & 56.44076 \\
\hline Virginia & 61.32657 \\
\hline Colorado & 62.02119 \\
\hline Nevada & 62.78103 \\
\hline New Jersey & 65.51067 \\
\hline New Mexico & 68.59366 \\
\hline Pennsylvania & 69.93497 \\
\hline Hawaii & 72.25409 \\
\hline Delaware & 74.05332 \\
\hline Montana & 76.1264 \\
\hline Utah & 91.15158 \\
\hline Alaska & 93.21341 \\
\hline Maryland & 97.37042 \\
\hline Maine & 111.5184 \\
\hline New Hampshire & 125.8811 \\
\hline Vermont & 131.2651 \\
\hline Massachusetts & 132.7475 \\
\hline Connecticut & 133.5888 \\
\hline Arizona & No Data \\
\hline
\end{tabular}

Notes: This table sorts states by Medicaid oxycodone prescriptions per 1,000 beneficiaries for 1991-1995. Triplicate states as of 1996 are bolded; former triplicate states are italicized. In a few circumstances, states are missing data for one or more quarters within a year. In these cases, we annualize the data within that year by multiplying the number of prescriptions by four divided by the number of quarters in the data. If a state is missing data for an entire year, we simply take the average over the years with data. 
Table A3: Initial State Oxycodone Prescribing Prevalence, 1995

\begin{tabular}{|c|c|}
\hline State & $\begin{array}{c}\text { Medicaid Prescriptions per } 1,000 \text { Benes } \\
(1995)\end{array}$ \\
\hline Texas & 1.437183 \\
\hline Illinois & 2.27907 \\
\hline California & 9.870143 \\
\hline Michigan & 9.947494 \\
\hline Kentucky & 12.64413 \\
\hline New York & 12.85399 \\
\hline Idaho & 17.5283 \\
\hline South Dakota & 17.94079 \\
\hline Indiana & 24.38553 \\
\hline Arkansas & 26.55574 \\
\hline Mississippi & 27.12157 \\
\hline Oregon & 29.42524 \\
\hline Minnesota & 30.09379 \\
\hline Iowa & 31.56931 \\
\hline Oklahoma & 34.66857 \\
\hline North Dakota & 34.84678 \\
\hline Alabama & 37.23684 \\
\hline Florida & 38.72538 \\
\hline Georgia & 39.09127 \\
\hline Rhode Island & 39.72491 \\
\hline South Carolina & 41.21292 \\
\hline Wyoming & 42.08354 \\
\hline Missouri & 42.2024 \\
\hline District Of Columbia & 43.54829 \\
\hline Kansas & 45.58431 \\
\hline Louisiana & 46.14725 \\
\hline North Carolina & 48.32907 \\
\hline Nebraska & 49.51213 \\
\hline West Virginia & 50.46479 \\
\hline Ohio & 50.68425 \\
\hline Nevada & 53.44331 \\
\hline New Jersey & 60.28119 \\
\hline Washington & 61.43809 \\
\hline Virginia & 63.08407 \\
\hline New Mexico & 63.87621 \\
\hline Wisconsin & 66.39986 \\
\hline Hawaii & 72.76386 \\
\hline Pennsylvania & 77.99833 \\
\hline Montana & 79.24383 \\
\hline Utah & 82.10937 \\
\hline Delaware & 88.18026 \\
\hline Alaska & 95.17448 \\
\hline Maryland & 114.2299 \\
\hline Vermont & 133.3962 \\
\hline Connecticut & 146.5896 \\
\hline Maine & 148.8184 \\
\hline Massachusetts & 156.795 \\
\hline New Hampshire & 157.518 \\
\hline Colorado & No Data \\
\hline Tennessee & No Data \\
\hline Arizona & No Data \\
\hline
\end{tabular}

Notes: This table sorts states by Medicaid oxycodone prescriptions per 1,000 beneficiaries for 1995. Triplicate states as of 1996 are bolded; former triplicate states are italicized. In a few circumstances, states are missing data for one or more quarters in 1995 . In these cases, we annualize the data within that year by multiplying the number of prescriptions by four divided by the number of quarters in the data. 


\section{B. Additional Robustness Tests}

We explore the robustness of our main results to including additional controls. First, Jaeger et al. (2018) suggest that it may be important in difference-in-differences analyses to permit the control variables to have different effects over time. We interact a subset of our covariates with year indicators to allow for this additional flexibility. ${ }^{1}$ The estimates, presented in Appendix Table B1, generally increase in magnitude when these interactions are included.

Next, we study the role of economic conditions and labor demand shocks. These results are included in Appendix Table B2. First, we include the annual unemployment rate (from the Bureau of Labor Statistics) as a control in Column (1). While this covariate is potentially endogenous if opioid misuse affects labor supply, the estimates are generally larger in magnitude. Next, we control for economic shocks that provide an exogenous source of variation in economic conditions. Charles et al. (2019) use a shift-share (Bartik) instrument to predict changes in manufacturing employment share, finding that reductions in manufacturing jobs increase drug overdose rates. We construct a shift-share instrument using the Current Population Study, fixing industry composition by state at its 1995 levels, and interacting these 1995 compositions with national-level industry-specific employment levels (excluding each state's own employment). Column (2) of Table B2 presents the results for overdose deaths per 100,000, controlling for this variable. The results are not meaningfully affected by the inclusion of this extra control. In Column (3), we add a shift-share instrument related to all industries (similar to Betz and Jones (2018)). The inclusion of both shift-share measures permits manufacturing shifts to have differential effects relative to broader labor demand shocks. Again, the results are similar.

Finally, Pierce and Schott (forthcoming) show that areas disproportionately harmed by international trade policy (specifically, the granting of Permanent Normal Trade Relations (PTNR) by the United States to China in 2000), experienced faster growth in fatal drug overdoses and other deaths of despair. We interact their metric of exposure to trade liberation with year indicators. The results are generally unaffected when we control for these variables. Columns (5)-(8) provide the same sensitivity tests for opioid overdose deaths.

In addition, we estimate our event study in equation (1) controlling for the PierceSchott measure of exposure to trade policy interacted with year fixed effects. Figure B1 shows the estimates for the non-triplicate interaction terms (Panels A and C) and the trade policy interaction terms (Panels B and D) estimated jointly. The non-triplicate pattern is unaffected by the inclusion of the trade exposure variable, suggesting that our main estimates are not driven by differential exposure to PTNR.

\footnotetext{
${ }^{1}$ We use only a subset since this approach adds a considerable amount of controls to the regression so there may be concerns of overfitting. We included covariates which varied substantially across triplicate and non-triplicate states in Table 1: fraction non-Hispanic white, fraction non-Hispanic black, fraction Hispanic, and $\log$ of population.
} 
Finally, in Table 1, we found notable differences in cocaine overdoses between triplicate and non-triplicate states for the baseline period 1991-1995. In Appendix Figure B2, we present event study estimates for cocaine overdoses, excluding opioids. We observe a transitory decline in cocaine overdoses in non-triplicate states relative to triplicate states prior to 1996. However, in general, we observe little evidence of any long-term relationship between triplicate status and cocaine overdoses, and it does not match the pattern observed for overdoses more generally or overdoses involving opioids.

Given the initial differences in cocaine overdoses, we test whether cocaine overdoses are confounding our estimates. In Appendix Figure B3, we present results for overdoses excluding cocaine (Panel A) and opioid-related overdoses excluding cocaine (Panel B). The results are generally unaffected by excluding cocaine. The estimates decrease in magnitude since we are excluding overdoses potentially impacted by OxyContin exposure, but the patterns are similar to the main event studies. In Panel C of Appendix Figure B3, we study opioid overdoses but exclude unspecified narcotics. Again, the results are generally unaffected. 
Figure B1: Event Study: Controlling for Pierce-Schott Trade Exposure Effect

\section{Overdose Deaths per 100,000}
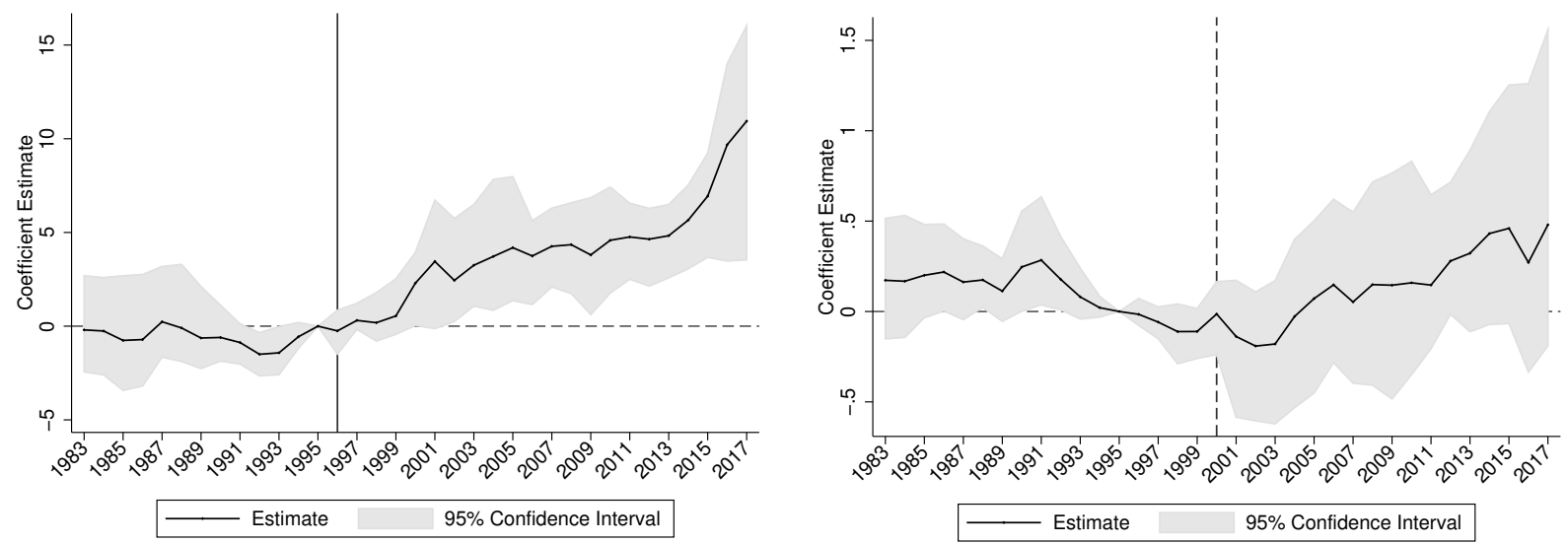

A: Non-Triplicate Effect

B: Trade Effect

\section{Opioid Overdose Deaths per 100,000}

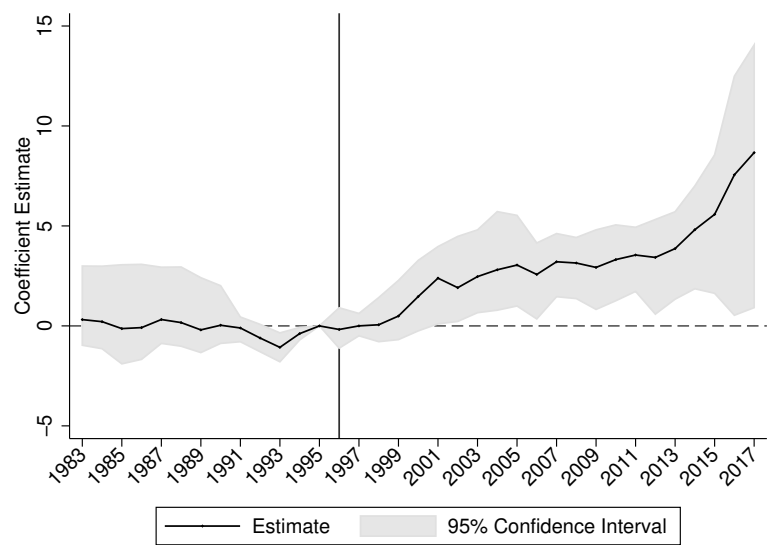

C: Non-Triplicate Effect

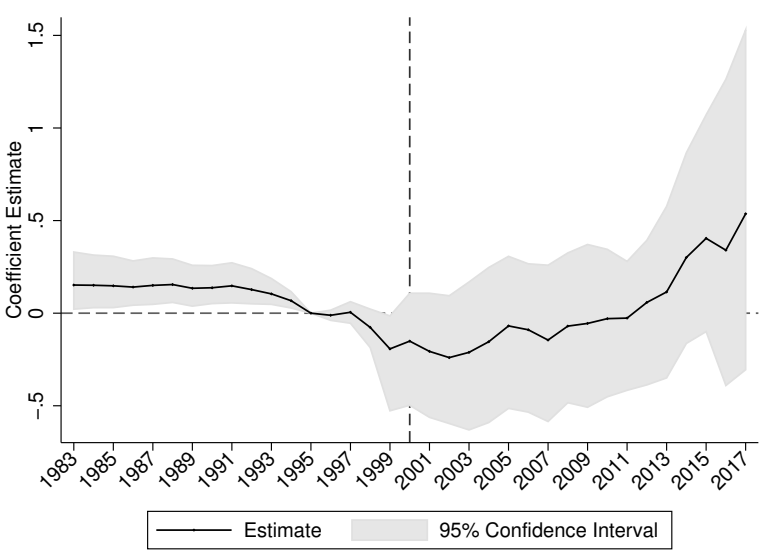

D: Trade Effect

Notes: We use geocoded NVSS data to construct overdose and opioid overdose deaths per 100,000. See text for exact ICD codes used in each period. Panels A and B are estimated jointly. Panel A shows the non-triplicate effect; Panel B shows the effect of exposure to trade liberalization. Panels C and D are also estimated jointly. Trade policy changed in 2000 and the exposure to the policy is defined in the same manner as Pierce and Schott (forthcoming). All regressions include state and year fixed effects. 95\% confidence intervals are generated using a clustered (at state) wild bootstrap. All estimates are normalized to 0 in 1995. 
Figure B2: Event Study: Cocaine Overdose Death Rates, Excluding Opioids

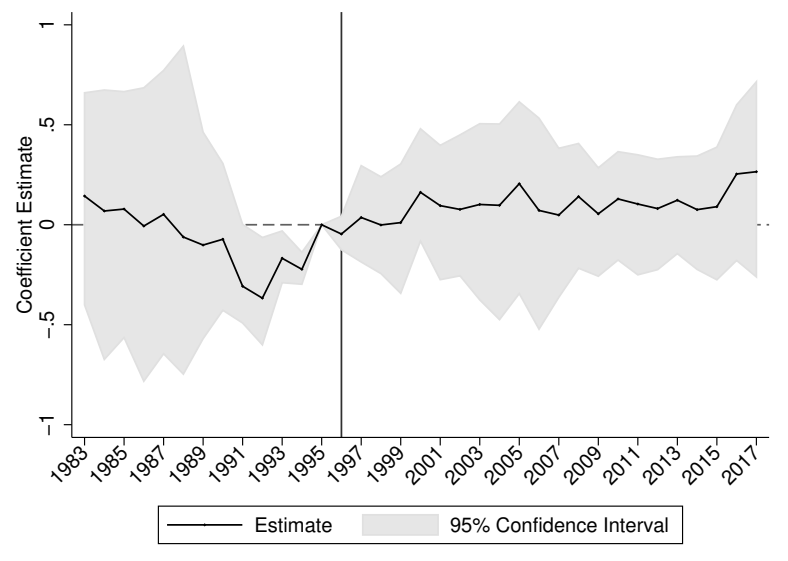

Notes: We use geocoded NVSS data to construct cocaine overdose deaths (excluding opioids) per 100,000. We report event study estimates from a regression which includes state and year fixed effects. $95 \%$ confidence intervals are generated using a clustered (at state) wild bootstrap. Estimates are normalized to 0 in 1995. 


\section{Figure B3: Event Studies: Excluding Cocaine or Unspecified Category}

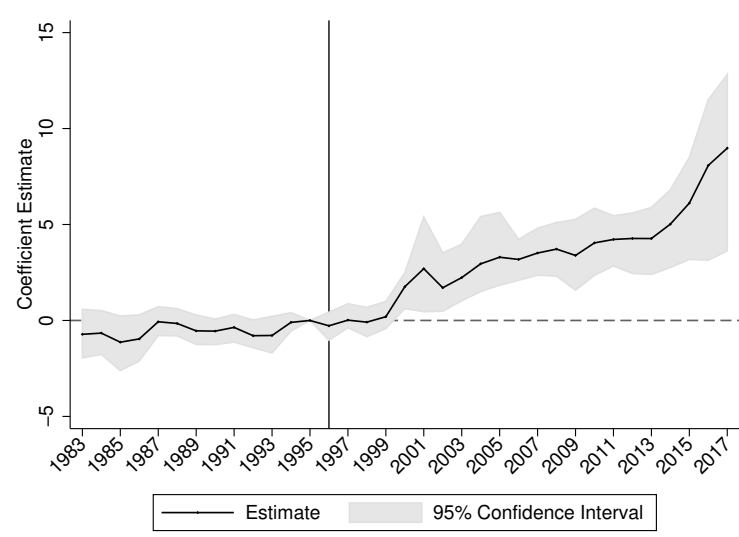

A: Overdose deaths excluding cocaine

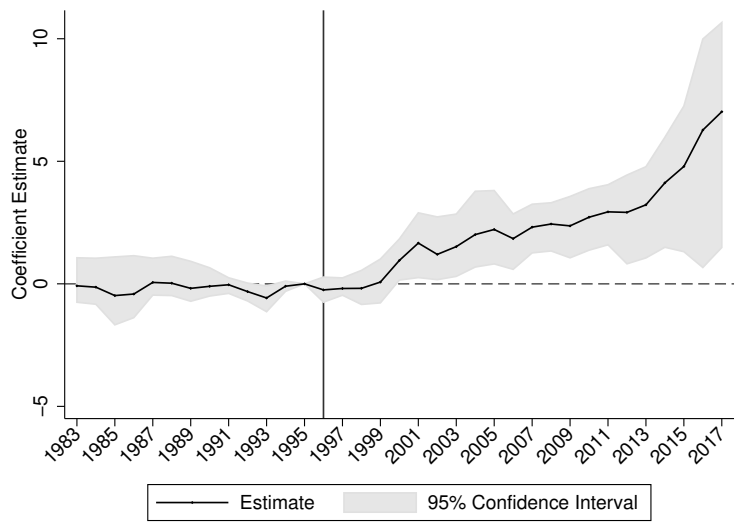

B: Opioid overdose deaths excluding cocaine

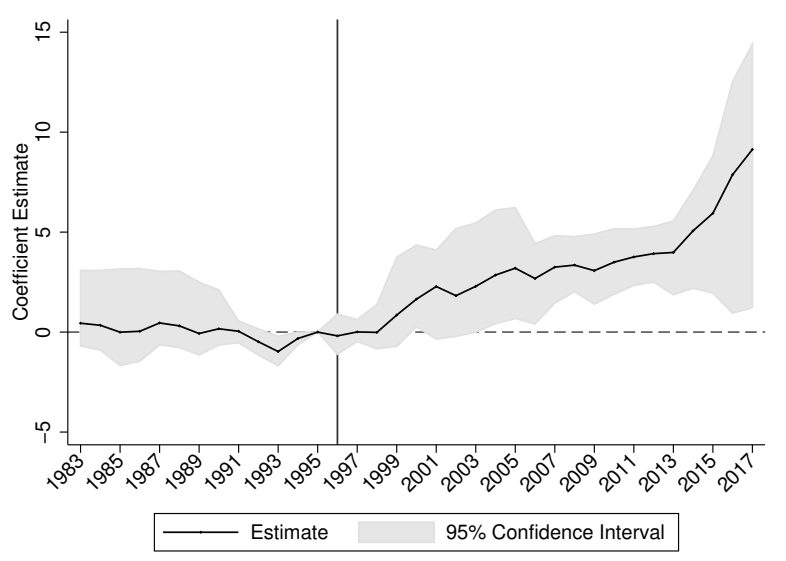

C: Opioid overdose deaths excluding T40.6

Notes: We use geocoded NVSS data to construct overdose deaths per 100,000. In Panel A and B, we exclude overdoses also involving cocaine. In Panel C, we study opioid-specific overdose deaths excluding unspecified narcotics (coded T40.6 in ICD-10). Event study estimates include state and year fixed effects. 95\% confidence intervals are generated using a clustered (at state) wild bootstrap. Estimates are normalized to 0 in 1995. 
Table B1: Difference-in-Differences Analyses: Interacting Covariates with Year Indicators

\begin{tabular}{ccc}
\hline $\begin{array}{c}\text { Outcome: } \\
\text { Non-Triplicate } \times\end{array}$ & $\begin{array}{c}\text { Overdoses } \\
(1)\end{array}$ & $\begin{array}{c}\text { Opioid Overdoses } \\
(2)\end{array}$ \\
\hline $1996-2000$ & $2.277^{* * *}$ & $1.442^{* *}$ \\
& {$[0.478,4.051]$} & {$[0.083,2.751]$} \\
$2001-2010$ & $5.747^{* *}$ & $3.558^{* *}$ \\
& {$[1.477,10.082]$} & {$[0.548,6.679]$} \\
$2011-2017$ & $6.769^{* * *}$ & $3.619^{* *}$ \\
& {$[3.938,10.339]$} & {$[0.678,7.141]$} \\
Joint P-Value & 0.009 & 0.143 \\
\hline
\end{tabular}

Notes: $N=1,377 .{ }^{* * *}$ Significance $1 \%,{ }^{* *}$ Significance $5 \%,{ }^{*}$ Significance $10 \%$. Outcomes are overdose and opioid overdose deaths per 100,000 people. The reported coefficients refer to the interaction of the given time period and an indicator for whether the state did not have a triplicate program in 1996. Estimates are relative to pre-period 1991-1995. 95\% confidence intervals reported in brackets are estimated by wild bootstrap. All models include state and year fixed effects. We also interact fraction non-Hispanic white, fraction non-Hispanic black, fraction Hispanic, and log of population with year indicators. This approach permits these covariates to have a different relationship with the outcomes in each year. We limit the covariates in these regressions to those which vary the most across the triplicate and non-triplicate states given that they are each interacted with year dummies. "Joint P-Value" refers to the p-value from a joint hypothesis test that all three non-triplicate post effects are equal to zero and is also estimated using a restricted wild bootstrap. 
Table B2: Difference-in-Differences Analyses: Controlling for Unemployment and Economic Shocks

\begin{tabular}{|c|c|c|c|c|}
\hline \multirow[b]{2}{*}{ Non-Triplicate $\times$} & \multicolumn{4}{|c|}{ Overdose Deaths per 100,000} \\
\hline & $(1)$ & $(2)$ & $(3)$ & $(4)$ \\
\hline \multirow[t]{2}{*}{$1996-2000$} & $1.004^{*}$ & $1.100^{* *}$ & $0.952^{*}$ & $1.154^{* *}$ \\
\hline & {$[-0.135,2.687]$} & {$[0.013,2.653]$} & {$[-0.055,2.436]$} & {$[0.204,2.620]$} \\
\hline \multirow[t]{2}{*}{ 2001-2010 } & $3.921^{* *}$ & $4.235^{* *}$ & $4.179 * *$ & $4.486 * *$ \\
\hline & {$[1.240,6.694]$} & {$[1.421,6.934]$} & {$[1.396,6.790]$} & {$[1.648,7.267]$} \\
\hline \multirow[t]{2}{*}{ 2011-2017 } & $6.770 * * *$ & $6.937^{* * *}$ & $6.841^{* * *}$ & $6.953^{* * *}$ \\
\hline & {$[4.384,8.689]$} & {$[4.408,8.878]$} & {$[4.342,8.771]$} & {$[4.536,8.876]$} \\
\hline \multirow[t]{2}{*}{ Joint P-Value } & 0.001 & 0.001 & 0.002 & 0.001 \\
\hline & \multicolumn{4}{|c|}{ Opioid Overdose Deaths per 100,000 } \\
\hline Non-Triplicate $\times$ & $(5)$ & $(6)$ & $(7)$ & $(8)$ \\
\hline \multirow[t]{2}{*}{$1996-2000$} & $0.814^{*}$ & $0.829^{*}$ & $0.857^{*}$ & $0.964^{* *}$ \\
\hline & {$[-0.136,1.871]$} & {$[-0.181,1.915]$} & {$[-0.051,1.939]$} & {$[0.163,2.009]$} \\
\hline \multirow[t]{2}{*}{$2001-2010$} & $2.750 * *$ & $2.762^{* *}$ & $2.773^{* *}$ & $3.096 * *$ \\
\hline & {$[0.205,4.974]$} & {$[0.050,4.917]$} & {$[0.046,4.951]$} & {$[0.453,5.316]$} \\
\hline \multirow[t]{2}{*}{ 2011-2017 } & $4.779 * * *$ & $4.791 * * *$ & $4.809 * * *$ & $4.889 * * *$ \\
\hline & {$[1.857,6.896]$} & {$[1.859,6.884]$} & {$[1.913,6.935]$} & {$[2.120,6.943]$} \\
\hline Joint P-Value & 0.017 & 0.020 & 0.017 & 0.011 \\
\hline Unemployment Rate & Yes & No & No & No \\
\hline Bartik Manufacturing & No & Yes & Yes & Yes \\
\hline Bartik All Industries & No & No & Yes & Yes \\
\hline Trade Exposure & No & No & No & Yes \\
\hline
\end{tabular}

Notes: $N=1,377 .{ }^{* * *}$ Significance $1 \%,{ }^{*}$ Significance $5 \%,{ }^{*}$ Significance $10 \%$. Outcomes are overdose and opioid overdose deaths per 100,000. The reported coefficients refer to the interaction of the given time period and an indicator for whether the state did not have a triplicate program in 1996. Estimates are relative to pre-period 1991-1995. 95\% confidence intervals reported in brackets are estimated by wild bootstrap. All models include state and year fixed effects as well as the fraction non-Hispanic white, fraction non-Hispanic black, fraction Hispanic, log of population, fraction with college degree, fraction ages 25-44, fraction ages 45-64, and fraction ages 65+. In Columns (1) and (5), we add the unemployment rate. In the rest of the columns, we include labor demand shocks. First, we include a shift-share instrument related specifically to manufacturing. Next, we also add a more general shift-share instrument which uses all industries. Finally, we also include a measure of exposure to trade interacted with year dummies. "Joint P-Value" refers to the p-value from a joint hypothesis test that all three non-triplicate post effects are equal to zero and is also estimated using a restricted wild bootstrap. 


\section{Alternative Inference Methods}

In this section, we consider the sensitivity of our results to alternative statistical inference methods. First, we show our main results with cluster-robust standard errors, the most commonly used method for accounting for serial correlation within states. This method may produce standard errors that are too small when there are too few clusters (or treated/untreated units). These results are presented in Appendix Table C1. As expected, confidence intervals are much tighter when using this traditional approach.

We also compute p-values using permutation-style tests. We randomly-assign triplicate status to 5 non-triplicate states and re-estimate equation (2). We repeat this procedure 10,000 times. In each permutation, we estimate the t-statistic for each of the three postperiods. Then we compare the placebo estimates to the non-triplicate t-statistic when the 5 triplicate states are correctly assigned and determine the rank. This approach is recommended in MacKinnon and Webb (forthcoming). In Appendix Figure C1, we show the distribution of the placebo t-statistics for each of the three time periods while marking the 2.5 and 97.5 percentiles with vertical dashed lines. The actual estimate is shown as a solid line. We also report the rank of this t-statistic (one-sided test) and the rank of the absolute value of the t-statistic (two-sided test). We find that it is statistically rare to observe our main overdose patterns for triplicate versus non-triplicate states using other combinations of states. For the earliest time period (1996-2000), the coefficient for non-triplicate states ranks 239 out of 10,000 (statistically significant at the 5\% level). For later time periods, the nontriplicate estimate ranks second and first out of 10,000, respectively. When we jointly test the estimates for the three time periods, we find that it is extremely rare to observe three t-statistics at the magnitude observed for our main effects. Appendix Figure C2 repeats this exercise but creates a distribution of placebo coefficient estimates instead of t-statistics. Again, the observed pattern of results is statistically rare. 
Figure C1: Permutation Tests using T-Statistics

Overdose Deaths per 100,000

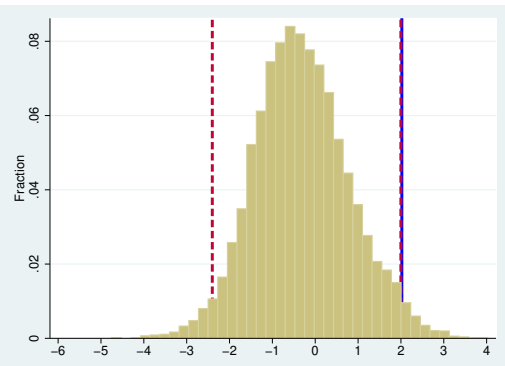

Rank of $T_{1}: 239 / 10,000$

Rank of $\left|T_{1}\right|: 743 / 10,000$

A: $1996-2000$

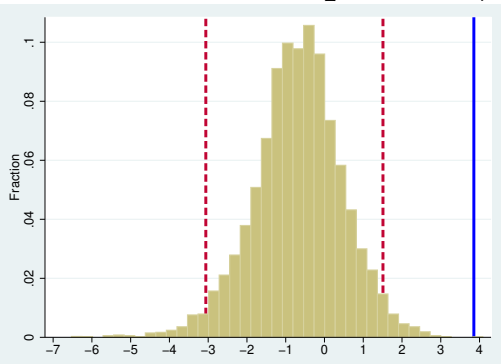

Rank of $T_{2}: 2 / 10,000$

Rank of $\left|T_{2}\right|: 78 / 10,000$

B: $2001-2010$

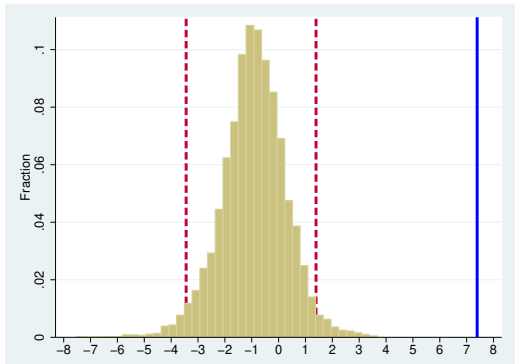

Rank of $T_{3}: 1 / 10,000$

Rank of $\left|T_{3}\right|: 3 / 10,000$

C: $2011-2017$

Joint Test (one-sided): $\hat{P}\left(T_{1}<T_{1}^{(k)}, T_{2}<T_{2}^{(k)}, T_{3}<T_{3}^{(k)}\right)=0.0000$

Joint Test (two-sided): $\hat{P}\left(\left|T_{1}\right|<\left|T_{1}^{(k)}\right|,\left|T_{2}\right|<\left|T_{2}^{(k)}\right|,\left|T_{3}\right|<\left|T_{3}^{(k)}\right|\right)=0.0000$

\section{Opioid Overdose Deaths per 100,000}

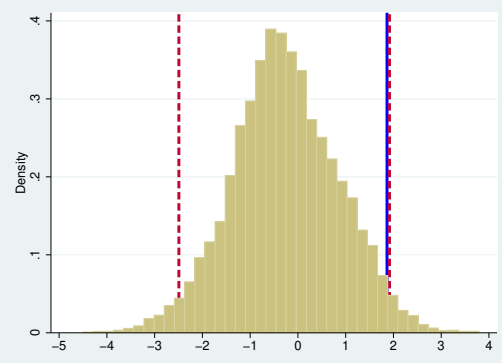

Rank of $T_{1}: 279 / 10,000$ Rank of $\left|T_{1}\right|: 1010 / 10,000$

D: $1996-2000$

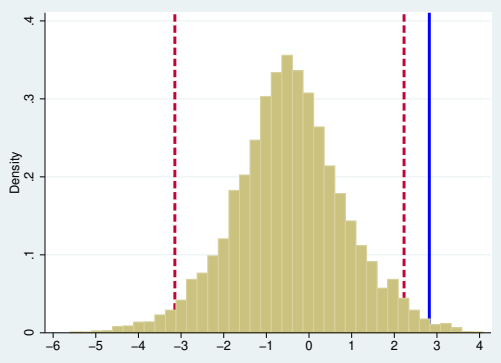

Rank of $T_{2}: 79 / 10,000$

Rank of $\left|T_{2}\right|: 478 / 10,000$

E: 2001-2010

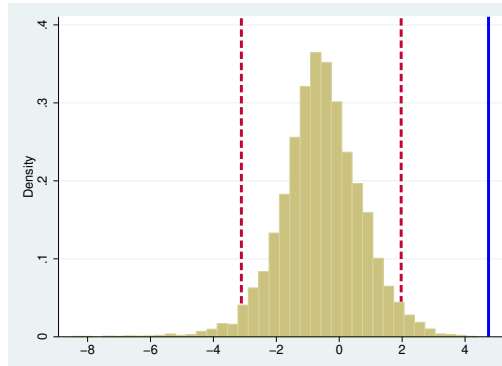

Rank of $T_{3}: 1 / 10,000$

Rank of $\left|T_{3}\right|: 50 / 10,000$

F: 2011-2017

Joint Test (one-sided): $\hat{P}\left(T_{1}<T_{1}^{(k)}, T_{2}<T_{2}^{(k)}, T_{3}<T_{3}^{(k)}\right)=0.0000$ Joint Test (two-sided): $\hat{P}\left(\left|T_{1}\right|<\left|T_{1}^{(k)}\right|,\left|T_{2}\right|<\left|T_{2}^{(k)}\right|,\left|T_{3}\right|<\left|T_{3}^{(k)}\right|\right)=0.0004$

Notes: The dashed vertical lines represent the 2.5 and 97.5 percentiles of the placebo t-statistics. The solid blue vertical line is the t-statistic when the five triplicate states are assigned correctly. The $\mathrm{x}$-axis represents the value of the t-statistics; the $\mathrm{y}$-axis represents the density. Estimating equation (2), regressions include state and time fixed effects as well as fraction non-Hispanic white, fraction non-Hispanic black, fraction Hispanic, log of population, fraction with college degree, fraction ages 25-44, fraction ages 45-64, and fraction ages 65+. t-statistics are calculated using clustered (by state) standard errors as recommended by MacKinnon and Webb (forthcoming). In the joint tests, $k$ indexes the placebo t-statistics. 
Figure C2: Permutation Tests using Coefficient Estimates

Overdose Deaths per 100,000

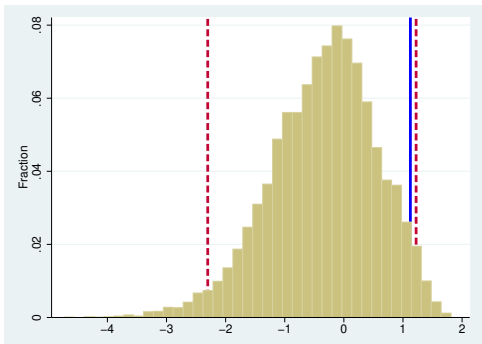

Rank of $\delta_{1}: 356 / 10,000$

Rank of $\left|\delta_{1}\right|: 2228 / 10,000$

A: $1996-2000$

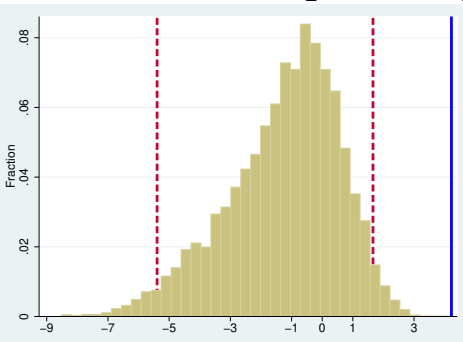

Rank of $\delta_{2}: 1 / 10,000$

Rank of $\left|\delta_{2}\right|: 760 / 10,000$

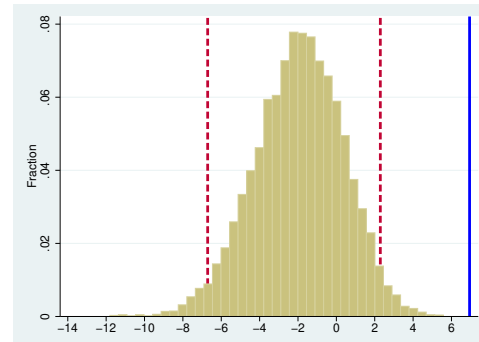

Rank of $\delta_{3}: 1 / 10,000$

Rank of $\left|\delta_{3}\right|: 188 / 10,000$

Joint Test (one-sided): $\hat{P}\left(\delta_{1}<\delta_{1}^{(k)}, \delta_{2}<\delta_{2}^{(k)}, \delta_{3}<\delta_{3}^{(k)}\right)=0.0000$

Joint Test (two-sided): $\hat{P}\left(\left|\delta_{1}\right|<\left|\delta_{1}^{(k)}\right|,\left|\delta_{2}\right|<\left|\delta_{2}^{(k)}\right|,\left|\delta_{3}\right|<\left|\delta_{3}^{(k)}\right|\right)=0.0053$

\section{Opioid Overdose Deaths per 100,000}

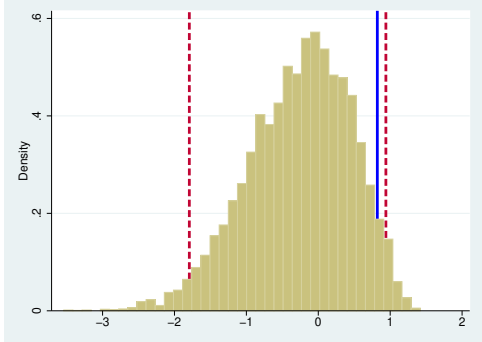

Rank of $\delta_{1}: 421 / 10,000$ Rank of $\left|\delta_{1}\right|: 2544 / 10,000$

D: $1996-2000$

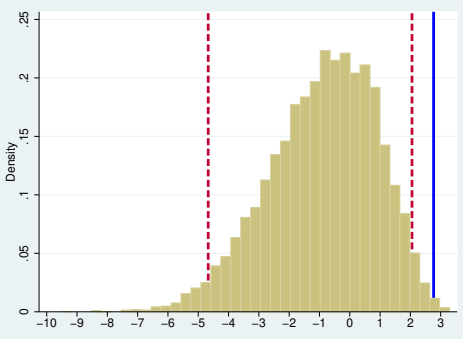

Rank of $\delta_{2}: 27 / 10,000$

Rank of $\left|\delta_{2}\right|: 1562 / 10,000$

E: 2001-2010

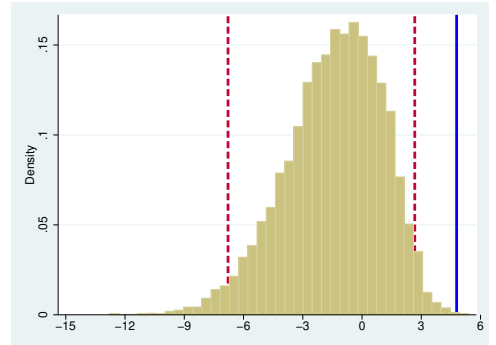

Rank of $\delta_{3}: 4 / 10,000$

Rank of $\left|\delta_{3}\right|: 939 / 10,000$

F: 2011-2017

Joint Test (one-sided): $\hat{P}\left(\delta_{1}<\delta_{1}^{(k)}, \delta_{2}<\delta_{2}^{(k)}, \delta_{3}<\delta_{3}^{(k)}\right)=0.0000$ Joint Test (two-sided): $\hat{P}\left(\left|\delta_{1}\right|<\left|\delta_{1}^{(k)}\right|,\left|\delta_{2}\right|<\left|\delta_{2}^{(k)}\right|,\left|\delta_{3}\right|<\left|\delta_{3}^{(k)}\right|\right)=0.0259$

Notes: The dashed vertical lines represent the 2.5 and 97.5 percentiles of the placebo estimates. The solid blue vertical line is the coefficent estimate when the five triplicate states are assigned correctly. The $\mathrm{x}$-axis represents the value of the coefficient estimates; the y-axis represents the density. Estimating equation (2), regressions include state and time fixed effects as well as fraction non-Hispanic white, fraction non-Hispanic black, fraction Hispanic, log of population, fraction with college degree, fraction ages 25-44, fraction ages $45-64$, and fraction ages $65+$. In the joint tests, $k$ indexes the placebo estimates. 
Table C1: Table 3 with Clustered (not bootstrapped) Confidence Intervals

\begin{tabular}{|c|c|c|c|c|}
\hline Non-Triplicate $\times$ & (1) & $\begin{array}{l}\text { Overdose Deat } \\
\text { (2) }\end{array}$ & s per 100,000 & $(4)$ \\
\hline \multirow[t]{2}{*}{$1996-2000$} & $1.244^{* * *}$ & $1.323^{* * *}$ & $1.125^{* *}$ & $1.452^{* * *}$ \\
\hline & {$[0.495,1.994]$} & {$[0.626,2.021]$} & {$[0.034,2.217]$} & {$[0.508,2.397]$} \\
\hline \multirow[t]{2}{*}{ 2001-2010 } & $3.758 * * *$ & $4.566^{* * *}$ & $4.221 * * *$ & $4.445^{* * *}$ \\
\hline & {$[1.918,5.597]$} & {$[2.856,6.275]$} & {$[2.074,6.368]$} & {$[2.976,5.913]$} \\
\hline \multirow[t]{2}{*}{ 2011-2017 } & $6.248^{* * *}$ & $7.903^{* * *}$ & $6.944^{* * *}$ & $6.816^{* * *}$ \\
\hline & {$[3.637,8.860]$} & {$[5.349,10.457]$} & {$[5.103,8.786]$} & {$[5.012,8.620]$} \\
\hline Weighted & No & Yes & Yes & Yes \\
\hline Covariates & No & No & Yes & Yes \\
\hline \multirow{2}{*}{$\begin{array}{c}\text { Region-Time Dummies } \\
\mathrm{N}\end{array}$} & No & No & No & Yes \\
\hline & 1,377 & 1,377 & 1,377 & 1,377 \\
\hline Non-Triplicate $\times$ & $(5)$ & $\begin{array}{c}\text { pioid Overdose I } \\
(6)\end{array}$ & $\begin{array}{l}\text { eaths per } 100,00 \\
(7)\end{array}$ & (8) \\
\hline \multirow[t]{2}{*}{$1996-2000$} & $0.702^{* *}$ & $0.631^{* *}$ & $0.821^{*}$ & $1.044^{* *}$ \\
\hline & {$[0.147,1.257]$} & {$[0.066,1.197]$} & {$[-0.041,1.684]$} & {$[0.232,1.856]$} \\
\hline \multirow[t]{2}{*}{$2001-2010$} & $2.675^{* * *}$ & $2.993 * * *$ & $2.766^{* * *}$ & $3.011 * * *$ \\
\hline & {$[1.320,4.030]$} & {$[1.689,4.297]$} & {$[0.844,4.689]$} & {$[1.490,4.532]$} \\
\hline \multirow[t]{2}{*}{$2011-2017$} & $5.133^{* * *}$ & $5.946^{* * *}$ & $4.789 * * *$ & $4.543^{* * *}$ \\
\hline & {$[2.381,7.885]$} & {$[3.036,8.855]$} & {$[2.811,6.766]$} & {$[2.700,6.386]$} \\
\hline Weighted & No & Yes & Yes & Yes \\
\hline Covariates & No & No & Yes & Yes \\
\hline Region-Time Dummies & No & No & No & Yes \\
\hline $\mathrm{N}$ & 1,377 & 1,377 & 1,377 & 1,377 \\
\hline
\end{tabular}

Notes: ***Significance 1\%, **Significance $5 \%$, *Significance $10 \%$. This table replicates Table 3 while reporting traditional clustered $95 \%$ confidence intervals instead of those generated by a wild bootstrap. The reported coefficients refer to the interaction of the given time period and an indicator for whether the state did not have a triplicate program in 1996. Estimates are relative to pre-period 1991-1995. All models include state and year fixed effects. Covariates include the fraction non-Hispanic white, fraction non-Hispanic black, fraction Hispanic, log of population, fraction with college degree, fraction ages 25-44, fraction ages 45-64, and fraction ages $65+$. 


\section{Synthetic Control Estimates}

While we observed little evidence of pre-existing trends in our results, the triplicate states did begin with higher levels of overdoses. One way to address differences in pre-treatment levels is to construct synthetic controls for each treated state using the synthetic control method (Abadie et al. $(2010,2015)){ }^{2}$ Here, we estimate synthetic controls for each triplicate state using non-triplicate states as potential components of the synthetic controls. In our difference-in-differences analyses, we aggregate overdoses to the annual level since all of our time-varying covariates only vary annually and since difference-in-differences only uses the (adjusted) means. Synthetic control estimation, however, benefits from the additional information in more disaggregated data (even if serially-correlated) so we use quarterly overdoses rates here. ${ }^{3}$

The "treatment" is triplicate state status in 1996 (unlike the prior analyses where the treatment was non-triplicate state status in 1996) since it makes more sense to use the 46 non-triplicate states to construct synthetic controls for the 5 triplicate states than vice versa. We then present the negative of the average weighted difference in the triplicate states relative to their synthetic controls, where the weights are the inverse of the variance in the pre-treatment period. This approach upweights states with more appropriate synthetic controls. The negative sign makes the estimates comparable to those presented throughout the paper. We also present the time series overdose rates for the triplicate and synthetic triplicate states, using these same weights. Thus, the time series trends will not match those shown earlier in the paper.

The results are shown in Figure D1. The synthetic control weights are shown in Table D2. We estimate similar overdose reductions as our main estimates. These estimates are summarized for our three aggregate time periods in Table D1. We multiply these estimates by 4 to make them comparable to the annual estimates in the main text. For inference, we use a permutation test, randomly-assigning triplicate status to non-triplicate states and then reporting the rank of the main estimate to the 999 placebo estimates. The estimates are similar to the difference-in-differences estimates and generally statistically significant at the $1 \%$ level. These results suggest that our main estimates are not driven by any initial outcome differences in overdose rates between the triplicate and non-triplicate states.

\footnotetext{
${ }^{2}$ Concerns about synthetic control estimation and some possible modifications are discussed in BenMichael et al. (2018); Arkhangelsky et al. (2019); Abadie (forthcoming); Powell (2018); Ferman and Pinto (2016); Doudchenko and Imbens (2016) among others. We use the traditional approach here.

${ }^{3}$ Given that we have a relatively long pre-period consisting of 52 quarters, we are less concerned about overfitting in this context and construct the synthetic controls based on the value of the outcome in each quarter in the pre-period.
} 
Figure D1: Synthetic Control Results: Overdose Deaths

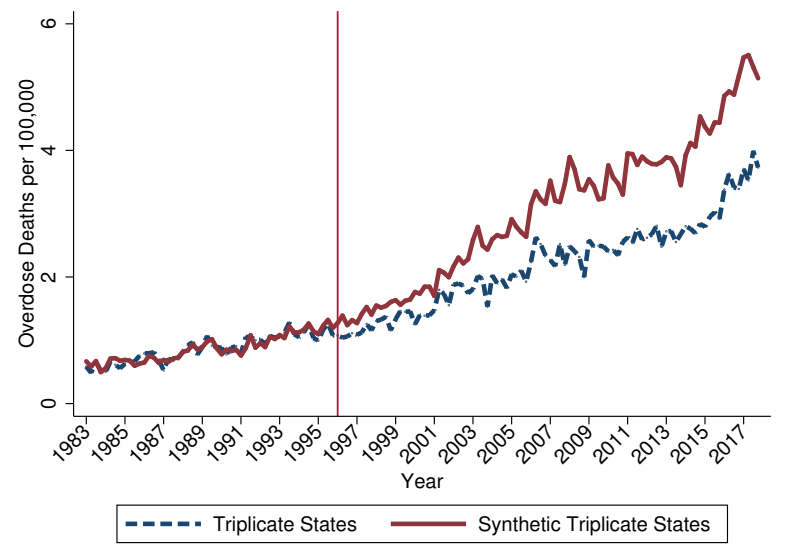

A: Triplicate and Synthetic Triplicate States

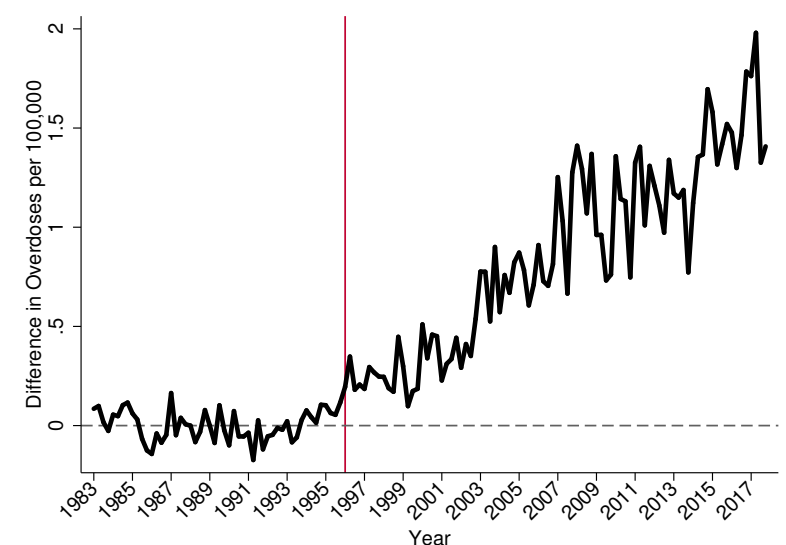

B: Difference

Synthetic Control Results: Opioid Overdose Deaths

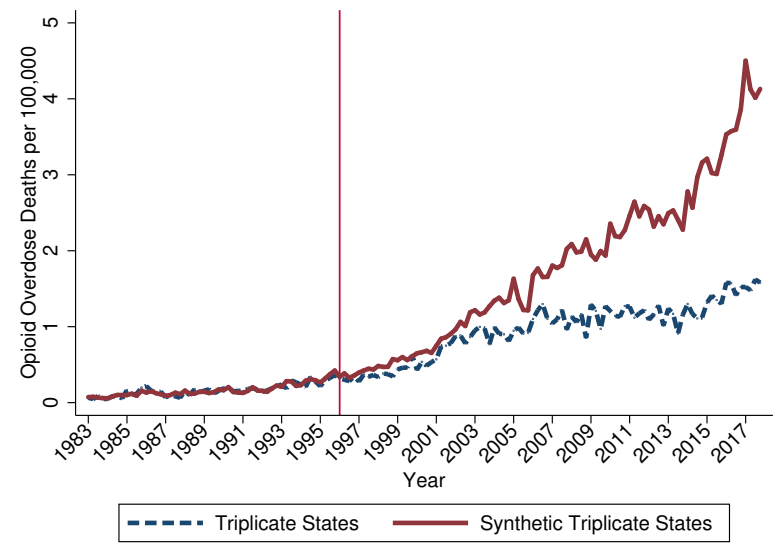

C: Triplicate and Synthetic Triplicate States

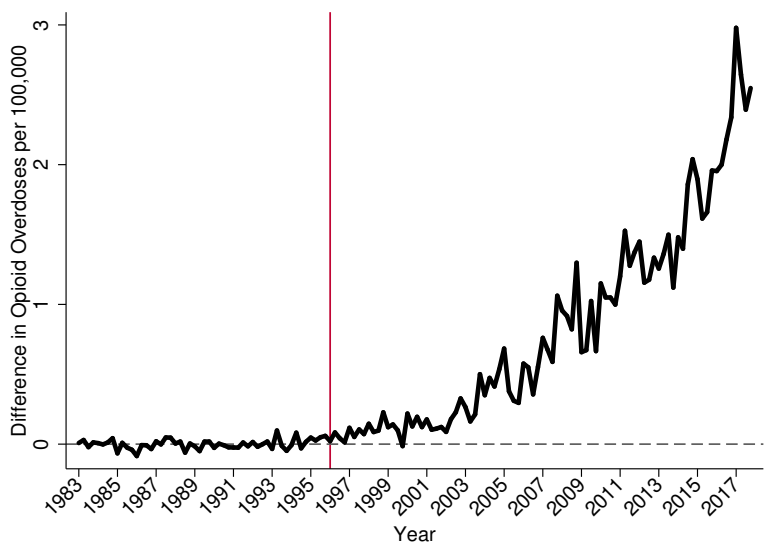

D: Difference

Notes: We construct a synthetic control for each triplicate state. We then take a weighted (by the "fit" of the synthetic control before 1996) average of the triplicate states and a weighted average of the synthetic control. These are plotted in Panel A (and C). The difference is shown in Panel B (and D). See Table D2 for the synthetic control weights. 
Table D1: Synthetic Control Results

\begin{tabular}{ccc}
\hline Non-Triplicate $\times$ & Overdoses per 100,000 & Opioid Overdoses per 100,000 \\
\hline $1996-2000$ & 1.099 & 0.415 \\
& {$[1 / 1000]$} & {$[149 / 1000]$} \\
$2001-2010$ & 3.201 & 2.230 \\
& {$[1 / 1000]$} & {$[8 / 1000]$} \\
$2011-2017$ & 5.405 & 6.954 \\
& {$[2 / 1000]$} & {$[1 / 1000]$} \\
\hline
\end{tabular}

Notes: We estimated synthetic controls for each triplicate state and report the weighted average of the synthetic control outcomes (which are non-triplicates) minus the triplicate state outcomes. We annualized the quarterly estimates by multiplying by four. The weights are the inverse of the variance before 1996. This approach considers the triplicate states as "treated" given that it would be difficult to construct synthetic controls for each non-triplicate state using only the 5 triplicate states. Below each estimate, we report the rank of that estimate relative to the 999 placebo estimates and the main estimate itself, produced by randomly-assigning states to "triplicate" status and repeating the entire strategy. We multiply the point estimates by four to make the quarterly estimates comparable to the annual estimates in the main text. 


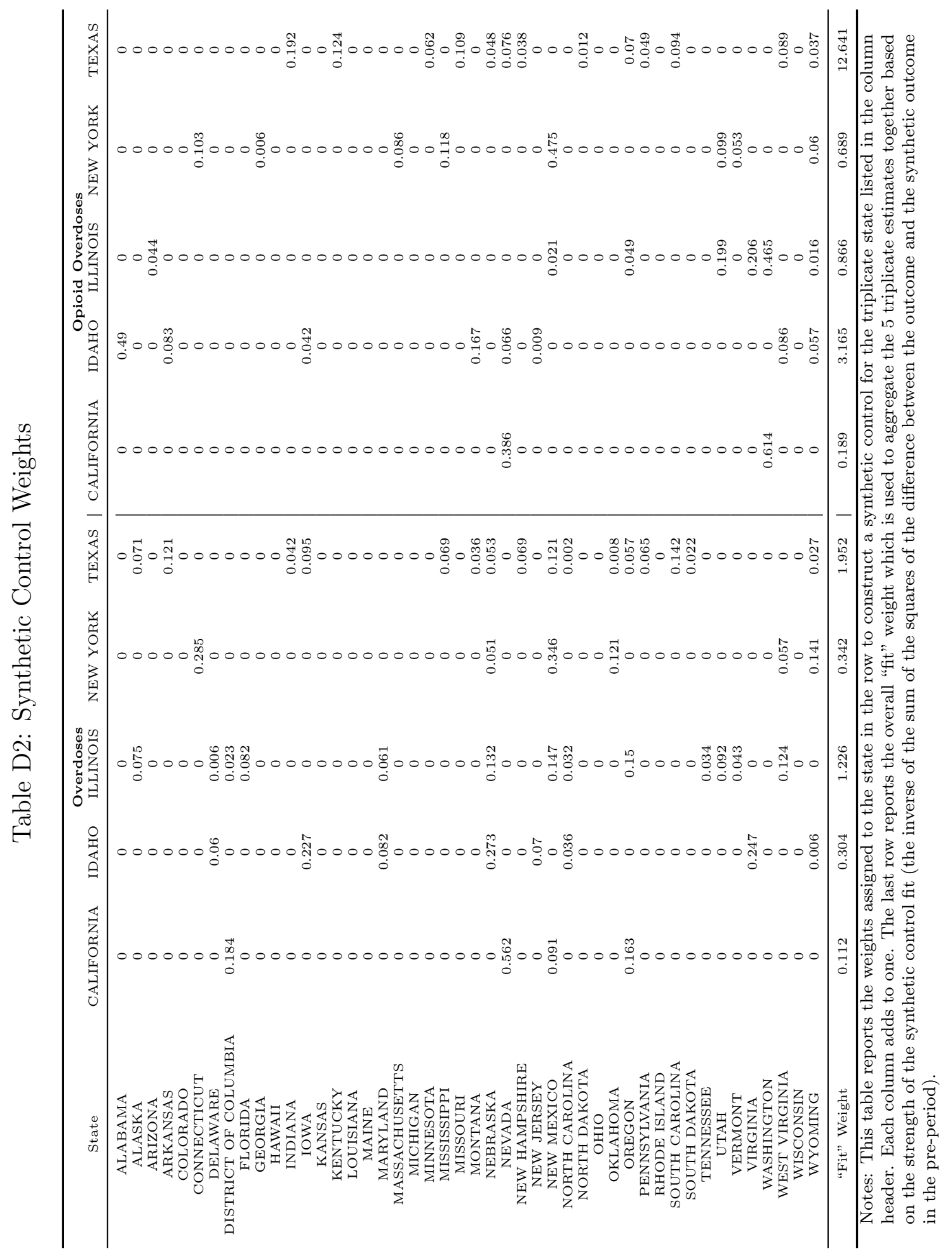




\section{Appendix References}

Abadie, Alberto, "Using Synthetic Controls: Feasibility, Data Requirements, and Methodological Aspects," Journal of Economic Literature, forthcoming.

_, Alexis Diamond, and Jens Hainmueller, "Synthetic control methods for comparative case studies: Estimating the effect of California's tobacco control program," Journal of the American Statistical Association, 2010, 105 (490), 493-505.

_ , _, and _ , "Comparative politics and the synthetic control method," American Journal of Political Science, 2015, 59 (2), 495-510.

Arkhangelsky, Dmitry, Susan Athey, David A Hirshberg, Guido W Imbens, and Stefan Wager, "Synthetic difference in differences," Technical Report, National Bureau of Economic Research 2019.

Ben-Michael, Eli, Avi Feller, and Jesse Rothstein, "New perspectives on the synthetic control method," Technical Report, Technical report, UC Berkeley 2018.

Betz, Michael R and Lauren E Jones, "Wage and employment growth in America's drug epidemic: Is all growth created equal?," American Journal of Agricultural Economics, 2018, 100 (5), 1357-1374.

Charles, Kerwin Kofi, Erik Hurst, and Mariel Schwartz, "The transformation of manufacturing and the decline in US employment," NBER Macroeconomics Annual, 2019, 33 (1), 307-372.

Doudchenko, Nikolay and Guido W Imbens, "Balancing, regression, difference-indifferences and synthetic control methods: A synthesis," Technical Report, National Bureau of Economic Research 2016.

Ferman, Bruno and Cristine Pinto, "Revisiting the synthetic control estimator," 2016.

Jaeger, David A, Theodore J Joyce, and Robert Kaestner, "A Cautionary Tale of Evaluating Identifying Assumptions: Did Reality TV Really Cause a Decline in Teenage Childbearing?," Journal of Business $\&$ Economic Statistics, 2018, pp. 1-10.

MacKinnon, James G and Matthew D Webb, "Randomization inference for differencein-differences with few treated clusters," Technical Report forthcoming.

Pardo, Bryce, "Do more robust prescription drug monitoring programs reduce prescription opioid overdose?," Addiction, 2017, 112 (10), 1773-1783.

Pierce, Justin $\mathbf{R}$ and Peter $\mathbf{K}$ Schott, "Trade liberalization and mortality: Evidence from US counties," American Economic Review: Insights, forthcoming.

Powell, David, "Imperfect Synthetic Controls," Technical Report, RAND 2018. 